\title{
Synthesis of Planar-Chiral Paracyclophanes via Samarium(II)-Catalyzed Intramolecular Pinacol Coupling
}

\author{
Tsuyoshi Ueda, Nobuhiro Kanomata, ${ }^{* \dagger}$ and Hajime Machida \\ Department of Industrial Chemistry, Meiji University, \\ Tama-ku, Kawasaki 214-8571, Japan \\ kanomata@waseda.jp
}

\section{Supplementary Information}

Full experimental procedures, characterization data of all new compounds,

${ }^{1} \mathrm{H}$ and ${ }^{13} \mathrm{C}$ NMR spectra of 2a-h, 3a-h, 4, 5c,d, $\mathbf{6 c}, \mathbf{d},\left(R_{\mathrm{p}}, \mathrm{S}\right)-$ and $\left(S_{\mathrm{p}}, S\right)-\mathbf{7 c}, \mathbf{d},\left(R_{\mathrm{p}}, \mathrm{S}\right)-$ and $\left(S_{\mathrm{p}}, S\right)-\mathbf{8 d}$, and $(R)-(+)-9 \mathbf{d}$, and ${ }^{1} \mathrm{H}$ NMR spectra of $(R)-(+)-$ and $(S)-(-)-9 \mathbf{c}$.

(81 Pages)

${ }^{\dagger}$ Present Address: Department of Chemistry, Waseda University, Shinjuku-ku, Tokyo 169-8555, Japan 
Table of Contents

ExperimentalSection

${ }^{1} \mathrm{H}$ NMR Spectrum of $\mathbf{2 a}$

${ }^{13} \mathrm{C}$ NMR Spectrum of $\mathbf{2 a}$

${ }^{1} \mathrm{H}$ NMR Spectrum of $\mathbf{2 b}$

${ }^{13} \mathrm{C}$ NMR Spectrum of $\mathbf{2 b}$

${ }^{1} \mathrm{H}$ NMR Spectrum of $\mathbf{2 c}$

${ }^{13} \mathrm{C}$ NMR Spectrum of $\mathbf{2 c}$

${ }^{1} \mathrm{H}$ NMR Spectrum of $\mathbf{2 d}$

${ }^{13} \mathrm{C}$ NMR Spectrum of $\mathbf{2 d}$

${ }^{1} \mathrm{H}$ NMR Spectrum of $\mathbf{2 e}$

${ }^{13} \mathrm{C}$ NMR Spectrum of $\mathbf{2 e}$

${ }^{1} \mathrm{H}$ NMR Spectrum of $\mathbf{2 f}$

${ }^{13} \mathrm{C}$ NMR Spectrum of $\mathbf{2 f}$

${ }^{1} \mathrm{H}$ NMR Spectrum of $\mathbf{2 g}$

${ }^{13} \mathrm{C}$ NMR Spectrum of $\mathbf{2 g}$

${ }^{1} \mathrm{H}$ NMR Spectrum of $\mathbf{2 h}$

${ }^{13} \mathrm{C}$ NMR Spectrum of $\mathbf{2 h}$

${ }^{1} \mathrm{H}$ NMR Spectrum of $\mathbf{3 a}$

${ }^{13} \mathrm{C}$ NMR Spectrum of $\mathbf{3 a}$

${ }^{1} \mathrm{H}$ NMR Spectrum of $\mathbf{3 b}$

${ }^{13} \mathrm{C}$ NMR Spectrum of $\mathbf{3 b}$

${ }^{1} \mathrm{H}$ NMR Spectrum of $\mathbf{3 c}$

${ }^{13} \mathrm{C}$ NMR Spectrum of $\mathbf{3 c}$

${ }^{1} \mathrm{H}$ NMR Spectrum of $\mathbf{3 d}$

${ }^{13} \mathrm{C}$ NMR Spectrum of $\mathbf{3 d}$

${ }^{1} \mathrm{H}$ NMR Spectrum of $\mathbf{3 e}$

${ }^{13} \mathrm{C}$ NMR Spectrum of $\mathbf{3 e}$

${ }^{1} \mathrm{H}$ NMR Spectrum of $\mathbf{3 f}$

${ }^{13} \mathrm{C}$ NMR Spectrum of $\mathbf{3 f}$
S3-S23

S24

S25

S26

S27

S28

S29

S30

S31

S32

S33

S34

S35

S36

S37

S38

S39

S40

S41

S42

S43

S44

$\mathrm{S} 45$

S46

S47

S48

S49

S50

S51
${ }^{1} \mathrm{H}$ NMR Spectrum of $\mathbf{3 g} \quad$ S52

${ }^{13} \mathrm{C}$ NMR Spectrum of $\mathbf{3 g} \quad$ S53

${ }^{1} \mathrm{H}$ NMR Spectrum of $\mathbf{3 h} \quad$ S54

${ }^{13} \mathrm{C}$ NMR Spectrum of $\mathbf{3 h} \quad \mathrm{S} 55$

${ }^{1} \mathrm{H}$ NMR Spectrum of $\mathbf{4}$ S56

${ }^{13} \mathrm{C}$ NMR Spectrum of $4 \quad$ S57

${ }^{1} \mathrm{H}$ NMR Spectrum of $\mathbf{5 c} \quad$ S58

${ }^{13} \mathrm{C}$ NMR Spectrum of $\mathbf{5 c} \quad$ S59

${ }^{1} \mathrm{H}$ NMR Spectrum of 5d $\quad$ S60

${ }^{13} \mathrm{C}$ NMR Spectrum of $\mathbf{5 d} \quad$ S61

${ }^{1} \mathrm{H}$ NMR Spectrum of $\mathbf{6 c} \quad$ S62

${ }^{13} \mathrm{C}$ NMR Spectrum of $\mathbf{6 c} \quad \mathrm{S} 63$

${ }^{1} \mathrm{H}$ NMR Spectrum of $\mathbf{6 d} \quad$ S64

${ }^{13} \mathrm{C}$ NMR Spectrum of $\mathbf{6 d} \quad$ S65

${ }^{1} \mathrm{H}$ NMR Spectrum of $\left(R_{\mathrm{p}}, \mathrm{S}\right)-7 \mathrm{c} \quad \mathrm{S} 66$

${ }^{13} \mathrm{C}$ NMR Spectrum of $\left(R_{\mathrm{p}}, \mathrm{S}\right)-7 \mathrm{c} \quad \mathrm{S} 67$

${ }^{1} \mathrm{H}$ NMR Spectrum of $\left(S_{\mathrm{p}}, S\right)-7 \mathrm{c} \quad \mathrm{S} 68$

${ }^{13} \mathrm{C}$ NMR Spectrum of $\left(S_{\mathrm{p}}, S\right)-7 \mathrm{c} \quad$ S69

${ }^{1} \mathrm{H}$ NMR Spectrum of $\left(R_{\mathrm{p}}, \mathrm{S}\right)-\mathbf{7 d} \quad \mathrm{S} 70$

${ }^{13} \mathrm{C}$ NMR Spectrum of $\left(R_{\mathrm{p}}, \mathrm{S}\right)-7 \mathbf{d} \quad \mathrm{S} 71$

${ }^{1} \mathrm{H}$ NMR Spectrum of $\left(S_{\mathrm{p}}, S\right)-\mathbf{7 d} \quad \mathrm{S} 72$

${ }^{13} \mathrm{C}$ NMR Spectrum of $\left(S_{\mathrm{p}}, S\right)-7 \mathbf{d} \quad S 73$

${ }^{1} \mathrm{H}$ NMR Spectrum of $\left(R_{\mathrm{p}}, \mathrm{S}\right)-\mathbf{8 d} \quad \mathrm{S} 74$

${ }^{13} \mathrm{C}$ NMR Spectrum of $\left(R_{\mathrm{p}}, \mathrm{S}\right)-\mathbf{8 d} \quad \mathrm{S} 75$

${ }^{1} \mathrm{H}$ NMR Spectrum of $\left(S_{\mathrm{p}}, S\right)$-8d $\quad$ S76

${ }^{13} \mathrm{C}$ NMR Spectrum of $\left(S_{\mathrm{p}}, S\right)-\mathbf{8 d} \quad \mathrm{S} 77$

${ }^{1} \mathrm{H}$ NMR Spectrum of $(R)-(+)-9 \mathrm{c} \quad \mathrm{S} 78$

${ }^{1} \mathrm{H}$ NMR Spectrum of $(S)-(-)-9 c \quad$ S79

${ }^{1} \mathrm{H}$ NMR Spectrum of $(R)-(+)-9 d \quad$ S80

${ }^{13} \mathrm{C}$ NMR Spectrum of $(R)-(+)-9 d \quad \mathrm{~S} 81$ 


\section{Experimental Section}

General. NMR spectra were recorded on JEOL JNM-GSX500, JNM-A400, or JNM-EX270 spectrometers. Chemical shifts are reported in ppm relative to $\mathrm{Me}_{4} \mathrm{Si}$ for ${ }^{1} \mathrm{H}$ NMR and to the central line of $\mathrm{CDCl}_{3}(77.0 \mathrm{ppm})$ for ${ }^{13} \mathrm{C} \mathrm{NMR}$ as internal standard. Unless otherwise specified, NMR spectra were measured at ambient temperature. Optical rotations were measured on JASCO P1010 polarimeters using a halogen lamp $(589 \mathrm{~nm})$. CD spectra were recorded using a Jasco J-820 spectropolarimeter. Mass spectra were recorded on JEOL JMS-700 (FAB) or JEOL JMS-SX102A (EI) spectrometer. All reactions were carried out in anhydrous solvents under argon atmosphere. Thin layer chromatography was performed using Merck silica gel $60 \mathrm{~F}_{254}$ glass plates (Art. 5715, $0.5 \mathrm{~mm}$ thick) for analytical purposes. Flash chromatography was performed using Silica gel FL60D (Fuji Silysia Chemical Ltd.) unless otherwise specified. Melting points were recorded on Yanagimoto apparatus and are uncorrected.

Double Sonogashira reactions. Representative procedure. To a solution of 1,4-diiodobenzene (1) $(0.5 \mathrm{~g}, 1.52 \mathrm{mmol})$ and $\mathrm{CuI}(87.5 \mathrm{mg}, 0.076 \mathrm{mmol})$ in $20 \mathrm{ml}$ of anhydrous deoxygenated $\mathrm{CH}_{2} \mathrm{Cl}_{2}-\mathrm{Et}_{3} \mathrm{~N}$ (1/3) was added 4-pentyn-1-ol (0.42 ml, 4.56 $\mathrm{mmol})$ and $\mathrm{Pd}\left(\mathrm{PPh}_{3}\right)_{4}(14.5 \mathrm{mg}, 0.076 \mathrm{mmol})$ and the mixture was stirred in the dark argon atmosphere at $50{ }^{\circ} \mathrm{C}$ for $24 \mathrm{~h}$. After the reaction was complete, ethyl acetate was added and the organic layer was washed with water and sat. $\mathrm{NH}_{4} \mathrm{Cl}$ aq. The aqueous layer was further extracted with ethyl acetate and the combined organic layer was dried over $\mathrm{MgSO}_{4}$. After removal of solvent in vacuo, the residue was separated by flash chromatography on silica gel with hexane-ethyl acetate (1/1) to give 1,4-bis(5-hydroxy-1-pentynyl)benzene in quantitative yield. 1,4-Bis(4-hydroxy-1-butynyl)benzene and 1,4-Bis(6-hydroxy-1-hexynyl)benzene were prepared by the reaction of 1 with 3-butyn-1-ol and 5-hexyn-1-ol, respectively. Methyl 2,5-bis(5-hydroxy-1-pentynyl)benzoate was also prepared by the reaction of methyl 2,5-dibromobenzoate with 4-pentyn-1-ol according to the similar procedure.

1,4-Bis(4-hydroxy-1-butynyl)benzene: $95 \%$ yield; white solid; mp 148.4-149.5 ${ }^{\circ} \mathrm{C}$; ${ }^{1} \mathrm{H}$ NMR (500 MHz, $\left.\mathrm{CDCl}_{3}\right) \delta=1.58(\mathrm{br}, 2 \mathrm{H}), 2.70(\mathrm{t}, J=6.3 \mathrm{~Hz}, 4 \mathrm{H}), 3.82$ $(\mathrm{t}, J=6.3 \mathrm{~Hz}, 4 \mathrm{H}), 7.33(\mathrm{~s}, 4 \mathrm{H}) ;{ }^{13} \mathrm{C}$ NMR $(125 \mathrm{MHz}$,

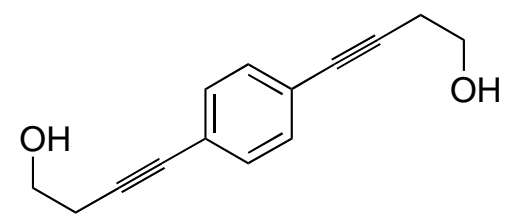
$\left.\mathrm{CDCl}_{3}\right) \delta=23.9(2 \mathrm{C}), 61.1(2 \mathrm{C}), 82.1(2 \mathrm{C}), 88.1(2 \mathrm{C}), 122.9$ (2C), 131.5 (4C); MS (EI): 
$m / z(\%): 214(82)\left[M^{+}\right], 69$ (100); high-resolution MS (EI, $\left.70 \mathrm{eV}\right)$ : calc. for $\mathrm{C}_{14} \mathrm{H}_{14} \mathrm{O}_{2}$ : 214.0994, found: 214.0999 .

1,4-Bis(5-hydroxy-1-pentynyl)benzene: quant. yield; white solid; mp 72.5-76.8 ${ }^{\circ} \mathrm{C} ;{ }^{1} \mathrm{H}$ NMR $\left(400 \mathrm{MHz}, \mathrm{CDCl}_{3}\right)$ $\delta=1.59(\mathrm{~s}, 2 \mathrm{H}), 1.86$ (quint, $J=6.5 \mathrm{~Hz}, 4 \mathrm{H}), 2.54(\mathrm{t}, J=$ $6.5 \mathrm{HZ}, 4 \mathrm{H}), 3.81(\mathrm{t}, J=6.5 \mathrm{~Hz}, 4 \mathrm{H}), 7.30(\mathrm{~s}, 4 \mathrm{H}) ;{ }^{13} \mathrm{C}$

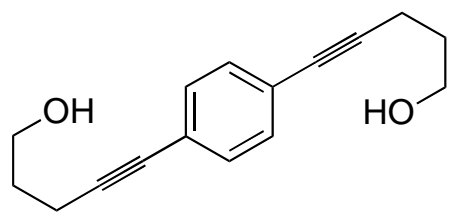
NMR (100 MHz, $\left.\mathrm{CDCl}_{3}\right) \delta=16.1$ (2C), 31.3 (2C), 61.8 (2C), 80.9 (2C), 90.9 (2C), 123.0 (2C), 131.4 (4C); high-resolution MS (EI, $70 \mathrm{eV}$ ): calc. for $\mathrm{C}_{16} \mathrm{H}_{18} \mathrm{O}_{2}$ : 242.1307, found: 242.1302 .

1,4-Bis(6-hydroxy-1-hexynyl)benzene: $88 \%$ yield; white solid; mp 69.9-72.1 ${ }^{\circ} \mathrm{C} ;{ }^{1} \mathrm{H}$ NMR $\left(500 \mathrm{MHz}, \mathrm{CDCl}_{3}\right) \delta=$ 1.31 (brs, 2H), $1.67-1.76(\mathrm{~m}, 8 \mathrm{H}), 2.46(\mathrm{t}, J=6.3 \mathrm{~Hz}, 4 \mathrm{H})$, $3.72(\mathrm{t}, J=6.3 \mathrm{~Hz}, 4 \mathrm{H}), 7.29(\mathrm{~s}, 4 \mathrm{H}) ;{ }^{13} \mathrm{C} \mathrm{NMR}(125 \mathrm{MHz}$,

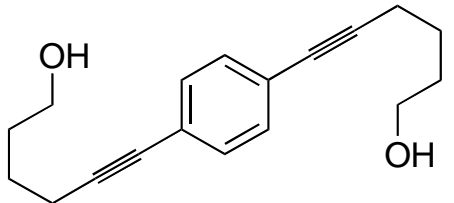
$\left.\mathrm{CDCl}_{3}\right) \delta=19.3$ (2C), 25.0 (2C), 31.9 (2C), 62.5 (2C), 80.7 (2C), 91.4 (2C), 123.1 (2C), 131.3 (4C); MS (EI): $\mathrm{m} / z(\%): 270$ (61) $\left[\mathrm{M}^{+}\right], 129$ (100); high-resolution MS (EI, 70 $\mathrm{eV}$ ): calc. for $\mathrm{C}_{18} \mathrm{H}_{22} \mathrm{O}_{2}: 270.1620$, found: 270.1615 .

Methyl 2,5-bis(5-hydroxy-1-pentynyl)benzoate: quant. yield; colorless oil; ${ }^{1} \mathrm{H}$ NMR $\left(500 \mathrm{MHz}, \mathrm{CDCl}_{3}\right) \delta=$ 1.81-1.92 (m, 4H), $2.54(\mathrm{t}, J=7.5 \mathrm{~Hz}, 2 \mathrm{H}), 2.62(\mathrm{t}, J=6.9$ $\mathrm{Hz}, 2 \mathrm{H}), 3.81(\mathrm{t}, J=6.3 \mathrm{~Hz}, 2 \mathrm{H}), 3.85(\mathrm{t}, J=5.7 \mathrm{~Hz}, 2 \mathrm{H})$,

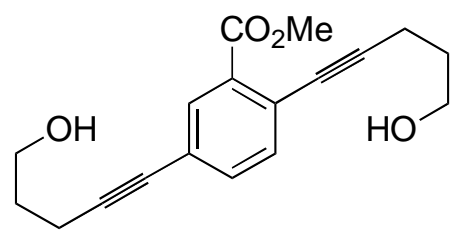
$3.90(\mathrm{~s}, 3 \mathrm{H}), 7.41(\mathrm{~d}, J=1.1 \mathrm{~Hz}, 2 \mathrm{H}), 7.92(\mathrm{~s}, 1 \mathrm{H}) ;{ }^{13} \mathrm{C} \mathrm{NMR}\left(125 \mathrm{MHz}, \mathrm{CDCl}_{3}\right) \delta=$ $15.9,16.6,30.8,31.2,52.3,61.4,61.6,79.7,79.8,92.0,96.6,123.0,123.4,132.0,133.4$, 134.0, 134.3, 166.2; MS (EI): $\mathrm{m} / z$ (\%): 300.1362 (14) $\left[M^{+}\right], 277$ (100); high-resolution MS (EI, $70 \mathrm{eV}$ ): calc. for $\mathrm{C}_{18} \mathrm{H}_{20} \mathrm{O}_{4}: 300.1362$, found: 300.1362 .

Stepwise Sonogashira reactions of 1-bromo-4-iodobenzene. Representative procedure. To a solution of 1-bromo-4-iodobenzene (20.0 g, $71.0 \mathrm{mmol})$ and CuI (406 $\mathrm{mg}, 2.13 \mathrm{mmol}$ ) in $320 \mathrm{ml}$ of anhydrous deoxygenated $\mathrm{CH}_{2} \mathrm{Cl}_{2}-\mathrm{Et}_{3} \mathrm{~N}$ (1/3) was added 4-pentyn-1-ol $(6.61 \mathrm{ml}, 71.0 \mathrm{mmol})$ and $\mathrm{Pd}\left(\mathrm{PPh}_{3}\right)_{4}(2.46 \mathrm{~g}, 2.13 \mathrm{mmol})$ and the mixture was stirred in the dark argon atmosphere at $50{ }^{\circ} \mathrm{C}$ for $24 \mathrm{~h}$. After the reaction was complete, ethyl acetate was added and the organic layer was washed with water and sat. $\mathrm{NH}_{4} \mathrm{Cl}$ aq. The aqueous layer was further extracted with ethyl acetate and the combined organic layer was dried over $\mathrm{MgSO}_{4}$. After removal of solvent in vacuo, the residue was 
separated by flash chromatography on silica gel with hexane-ethyl acetate (3/1) to give 5-(4-bromophenyl)-4-pentyn-1-ol (16.04 g, 94\%). 4-(4-Bromophenyl)-3-butyn-1-ol was prepared by the reaction with 3-butyn-1-ol according to the similar procedure.

4-(4-Bromophenyl)-3-butyn-1-ol: quant. yield; white solid; mp 66.7-69.2 ${ }^{\circ} \mathrm{C} ;{ }^{1} \mathrm{H}$ NMR (400 MHz, $\left.\mathrm{CDCl}_{3}\right) \delta=2.68(\mathrm{t}, J=$ $6.0 \mathrm{~Hz}, 2 \mathrm{H}), 3.82(\mathrm{t}, J=6.0 \mathrm{~Hz}, 2 \mathrm{H}), 7.27(\mathrm{~d}, J=8.0 \mathrm{~Hz}, 2 \mathrm{H})$,

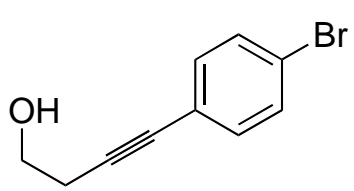
$7.42(\mathrm{~d}, J=8.0 \mathrm{~Hz}, 2 \mathrm{H}) ;{ }^{13} \mathrm{C} \mathrm{NMR}\left(100 \mathrm{MHz}, \mathrm{CDCl}_{3}\right) \delta=23.8$, 61.0, 81.4, 87.7, 122.1, 122.3, 131.5 (2C), 133.1 (2C); high-resolution MS (EI, $70 \mathrm{eV})$ : calc. for $\mathrm{C}_{10} \mathrm{H}_{9}{ }^{79} \mathrm{BrO}$ : 223.9837, found: 223.9837 .

5-(4-Bromophenyl)-4-pentyn-1-ol: $94 \%$ yield; white solid; mp 46.2-46.5 ${ }^{\circ} \mathrm{C} ;{ }^{1} \mathrm{H}$ NMR $\left(500 \mathrm{MHz}, \mathrm{CDCl}_{3}\right) \delta=1.69$ (br, $\left.1 \mathrm{H}\right)$, 1.86 (quint, $J=6.3 \mathrm{~Hz}, 2 \mathrm{H}), 2.52(\mathrm{t}, J=6.3 \mathrm{~Hz}, 2 \mathrm{H}), 3.80$ (t, $J=$

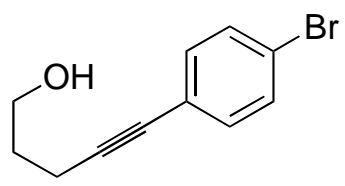
$6.3 \mathrm{~Hz}, 2 \mathrm{H}), 7.25(\mathrm{~d}, J=7.5 \mathrm{~Hz}, 2 \mathrm{H}), 7.41(\mathrm{~d}, J=7.5 \mathrm{~Hz}, 2 \mathrm{H})$;

${ }^{13} \mathrm{C} \mathrm{NMR}\left(125 \mathrm{MHz}, \mathrm{CDCl}_{3}\right) \delta=16.0,31.3,61.7,80.1,90.7,121.8,122.7,131.4(2 \mathrm{C})$, 133.0 (2C); high-resolution MS (EI, $70 \mathrm{eV}$ ): calc. for $\mathrm{C}_{11} \mathrm{H}_{11}{ }^{79} \mathrm{BrO}$ : 237.9993, found: 237.9991.

The second Sonogashira reactions of the compounds obtained were carried out according to the similar procedure mentioned above to give the corresponding diynediol products in quantitative yields. 5-(4-(4-Hydroxy-1-butynyl)phenyl)-4-pentyn-1-ol and 6-(4-(5-hydroxy-1-pentynyl)phenyl)-5-hexyn-1-ol were prepared by the reactions of 5-(4-bromophenyl)-4-pentyn-1-ol with 3-butyn-1-ol and 5-hexyn-1-ol, respectively. Similarly, 6-(4-(4-hydroxy-1-butynyl)phenyl)-5-hexyn-1-ol was prepared by the reaction of 4-(4-bromophenyl)-3-butyn-1-ol with 5-hexyn-1-ol.

\section{5-(4-(4-Hydroxy-1-butynyl)phenyl)-4-pentyn-1-ol:}

94\% yield; colorless oil; ${ }^{1} \mathrm{H}$ NMR $\left(500 \mathrm{MHz}, \mathrm{CDCl}_{3}\right) \delta=$ 1.69 (br, 2H), 1.86 (quint, $J=7.0 \mathrm{~Hz}, 2 \mathrm{H}), 2.51-2.57$ (m, $4 \mathrm{H}), 3.79-3.85(\mathrm{~m}, 4 \mathrm{H}), 7.29-7.33(\mathrm{~m}, 4 \mathrm{H}) ;{ }^{13} \mathrm{C} \mathrm{NMR}$

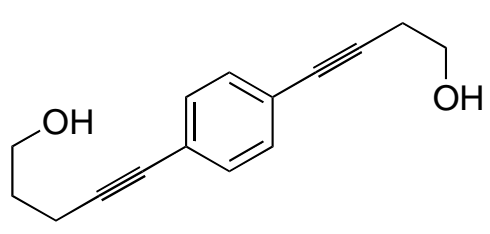
$\left(125 \mathrm{MHz}, \mathrm{CDCl}_{3}\right) \delta=15.9,23.4,31.2,60.6,61.0,80.7,81.9,88.0,91.1,122.5,123.2$, 131.3 (2C), 131.4 (2C); MS (EI): $m / z(\%): 228$ (1) $\left[M^{+}\right], 77$ (100); high-resolution MS (EI, $70 \mathrm{eV}$ ): calc. for $\mathrm{C}_{15} \mathrm{H}_{16} \mathrm{O}_{2}: 228.1150$, found: 228.1158 . 


\section{6-(4-(5-Hydroxy-1-pentynyl)phenyl)-5-hexyn-1-ol:}

quant. yield; colorless oil; ${ }^{1} \mathrm{H}$ NMR $\left(500 \mathrm{MHz}, \mathrm{CDCl}_{3}\right) \delta$ $=1.59-1.87(\mathrm{~m}, 6 \mathrm{H}), 2.46(\mathrm{t}, J=7.0 \mathrm{~Hz}, 2 \mathrm{H}), 2.53(\mathrm{t}, J=$ $7.0 \mathrm{~Hz}, 2 \mathrm{H}), 3.68-3.71(\mathrm{~m}, 2 \mathrm{H}), 3.79-3.82(\mathrm{~m}, 2 \mathrm{H}), 7.29$

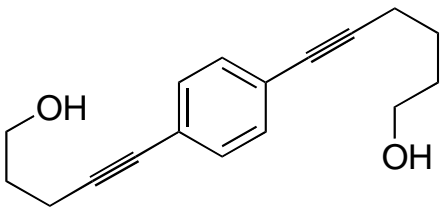
(s, 2H), 7.30 (s, 2H); ${ }^{13} \mathrm{C}$ NMR $\left(125 \mathrm{MHz}, \mathrm{CDCl}_{3}\right) \delta=16.0,19.2,24.9,31.3,31.8,62.2$, 62.3, 80.6, 80.8, 90.9, 91.5, 122.9, 123.1, 131.3 (2C), 131.3 (2C); high-resolution MS (EI, $70 \mathrm{eV}$ ): calc. for $\mathrm{C}_{17} \mathrm{H}_{20} \mathrm{O}_{2}: 256.1463$, found: 256.1480 .

\section{6-(4-(4-Hydroxy-1-butynyl)phenyl)-5-hexyn-1-ol:}

quant. yield; colorless syrup; ${ }^{1} \mathrm{H}$ NMR $(400 \mathrm{MHz}$, $\left.\mathrm{CDCl}_{3}\right) \delta=1.65-1.69(\mathrm{~m}, 4 \mathrm{H}), 2.39(\mathrm{t}, J=6.5 \mathrm{~Hz}, 2 \mathrm{H})$, $2.63(\mathrm{t}, J=6.5 \mathrm{~Hz}, 2 \mathrm{H}), 3.64(\mathrm{t}, J=6.5 \mathrm{~Hz}, 2 \mathrm{H}), 3.74(\mathrm{t}, J$

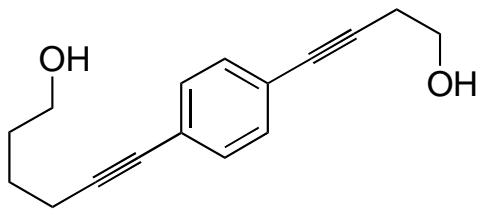
$=6.5 \mathrm{~Hz}, 2 \mathrm{H}), 7.24-7.25(\mathrm{~m}, 4 \mathrm{H}) ;{ }^{13} \mathrm{C} \mathrm{NMR}\left(100 \mathrm{MHz}, \mathrm{CDCl}_{3}\right) \delta=19.3,23.9,24.9$, 31.9, 61.1, 62.5, 80.7, 82.2, 87.8, 91.7, 122.5, 123.5, 131.4 (2C), 131.5 (2C); MS (EI): $m / z$ (\%): 242 (100) $\left[M^{+}\right]$; high-resolution MS (EI, $70 \mathrm{eV}$ ): calc. for $\mathrm{C}_{16} \mathrm{H}_{18} \mathrm{O}_{2}$ : 242.1307, found: 242.1316.

Hydrogenation reactions of diynediols. Representative procedure. A solution of 1,4-bis(5-hydroxy-1-pentynyl)benzene $(376 \mathrm{mg})$ in ethyl acetate $(20 \mathrm{ml})$ was stirred in the presence of $10 \%-\mathrm{Pd} / \mathrm{C}(38 \mathrm{mg})$ in hydrogen atmosphere at room temperature for 12 h. After the reaction was complete, the mixture was filtered though celite and the filtrate was concentrated in vacuo. The residue was separated by flash chromatography on silica gel with hexane-ethyl acetate (1/1) to give 1,4-bis(5-hydroxypentyl)benzene (364 $\mathrm{mg}, 96 \%$ ). The other diols were prepared from the corresponding diynediols according to the similar procedure.

1,4-Bis(4-hydroxybutyl)benzene: quant. yield; white solid; m.p. 57.4-58.3 ${ }^{\circ} \mathrm{C} ;{ }^{1} \mathrm{H}$ NMR $\left(500 \mathrm{MHz}, \mathrm{CDCl}_{3}\right)$

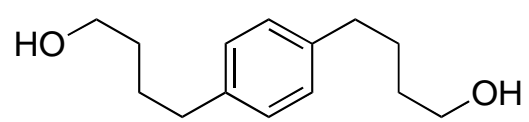
$\delta=1.35(\mathrm{br}, 2 \mathrm{H}), 1.57-1.71(\mathrm{~m}, 8 \mathrm{H}), 2.61(\mathrm{t}, J=6.8$ $\mathrm{Hz}, 4 \mathrm{H}), 3.65(\mathrm{t}, J=6.3 \mathrm{~Hz}, 4 \mathrm{H}), 7.09(\mathrm{~s}, 4 \mathrm{H}) ;{ }^{13} \mathrm{C} \mathrm{NMR}\left(125 \mathrm{MHz}, \mathrm{CDCl}_{3}\right) \delta=27.6$ (2C), 32.4 (2C), 35.2 (2C), 62.9 (2C), 128.3 (4C), 139.7 (2C); MS (EI): $m / z(\%): 222$ (75) $\left[M^{+}\right], 117$ (100); high-resolution MS (EI, $70 \mathrm{eV}$ ): calc. for $\mathrm{C}_{14} \mathrm{H}_{22} \mathrm{O}_{2}: 222.1620$, found: 222.1628. 


\section{5-(4-(4-Hydroxybutyl)phenyl)pentan-1-ol: $\quad 94 \%$}

yield; colorless oil; ${ }^{1} \mathrm{H}$ NMR $\left(500 \mathrm{MHz}, \mathrm{CDCl}_{3}\right) \delta=$  1.35-1.41 (m, 2H), 1.53-1.61 (m, 8H), 1.72 (brs, $2 \mathrm{H})$, 2.54-2.61 (m, 4H), 3.59-3.65 (m, 4H), $7.06(\mathrm{~s}, 4 \mathrm{H}) ;{ }^{13} \mathrm{C}$ NMR $\left(125 \mathrm{MHz}, \mathrm{CDCl}_{3}\right) \delta=$ 25.4, 27.6, 31.3, 32.3, 32.6, 35.2, 35.4, 62.8, 62.9, 128.29 (2C), 128.31 (2C), 139.6, 140.0; high-resolution MS (EI, $70 \mathrm{eV}$ ): calc. for $\mathrm{C}_{15} \mathrm{H}_{24} \mathrm{O}_{2}: 236.1176$, found: 236.1777 .

1,4-Bis(5-hydroxypentyl)benzene: $96 \%$ yield; white solid; m.p. 42.0-43.5 ${ }^{\circ} \mathrm{C} ;{ }^{1} \mathrm{H}$ NMR (400 MHz, $\left.\mathrm{CDCl}_{3}\right) \delta$ $=1.36-1.43(\mathrm{~m}, 4 \mathrm{H}), 1.56-1.67(\mathrm{~m}, 8 \mathrm{H}), 2.59(\mathrm{t}, J=6.0$

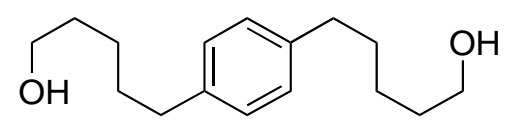
$\mathrm{Hz}, 4 \mathrm{H}), 3.63(\mathrm{t}, J=6.0 \mathrm{~Hz}, 4 \mathrm{H}), 7.09(\mathrm{~s}, 4 \mathrm{H}) ;{ }^{13} \mathrm{C} \mathrm{NMR}\left(100 \mathrm{MHz}, \mathrm{CDCl}_{3}\right) \delta=25.4$ (2C), 31.3 (2C), 32.6 (2C), $35.5 \quad$ (2C), 62.9 (2C), 128.3 (4C), 139.8 (2C); high-resolution MS (EI, $70 \mathrm{eV}$ ): calc. for $\mathrm{C}_{16} \mathrm{H}_{26} \mathrm{O}_{2}: 250.1933$, found: 250.1925 .

6-(4-(4-Hydroxybutyl)phenyl)hexan-1-ol: quant. yield; colorless syrup; ${ }^{1} \mathrm{H}$ NMR (400 MHz, $\mathrm{CDCl}_{3}$ ) $\delta=1.25-1.32(\mathrm{~m}, 4 \mathrm{H}), 1.45-1.64(\mathrm{~m}, 8 \mathrm{H}), 2.48-2.55$

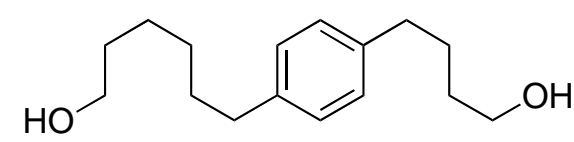
(m, 4H), 3.53-3.59 (m, 4H), $7.01(\mathrm{~s}, 4 \mathrm{H}),{ }^{13} \mathrm{C}$ NMR $\left(100 \mathrm{MHz}, \mathrm{CDCl}_{3}\right) \delta=25.6,27.5$, 29.0, 31.4, 32.3, 32.6, 35.2, 35.4, 62.8, 62.9, 128.2 (2C), 128.3 (2C), 139.5, 140.0; high-resolution MS (EI, $70 \mathrm{eV}$ ): calc. for $\mathrm{C}_{16} \mathrm{H}_{26} \mathrm{NaO}_{2}: 273.1831$, found: 273.1838 . 6-(4-(5-Hydroxypentyl)phenyl)hexan-1-ol:
yield; colorless oil; ${ }^{1} \mathrm{H}$ NMR $\left(500 \mathrm{MHz}, \mathrm{CDCl}_{3}\right) \delta=$ $1.28-1.66(\mathrm{~m}, 14 \mathrm{H}), 2.57(\mathrm{t}, J=7.5 \mathrm{~Hz}, 4 \mathrm{H})$,

3.56-3.69 (m, 4H), $7.08(\mathrm{~s}, 4 \mathrm{H}) ;{ }^{13} \mathrm{C} \mathrm{NMR}\left(125 \mathrm{MHz}, \mathrm{CDCl}_{3}\right) \delta=25.4,25.5,29.0,31.3$, $31.4,32.55,32.60,35.38,35.40,62.77,62.82,128.20$ (2C), 128.21 (2C), 139.7, 139.9; high-resolution MS (EI, $70 \mathrm{eV}$ ): calc. for $\mathrm{C}_{17} \mathrm{H}_{28} \mathrm{O}_{2}: 264.2089$, found: 264.2086 .

1,4-Bis(6-hydroxyhexyl)benzene: quant. yield; white solid; m.p. $42.1-43.7{ }^{\circ} \mathrm{C} ;{ }^{1} \mathrm{H}$ NMR (500 $\left.\mathrm{MHz}, \mathrm{CDCl}_{3}\right) \delta=1.28$ (brs, $2 \mathrm{H}$ ), 1.36-1.38 (m,

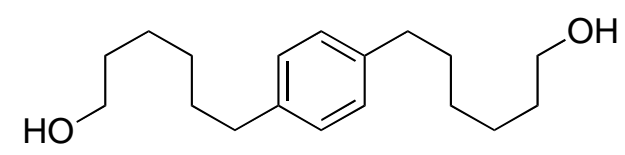
$8 \mathrm{H}$ ), 1.56 (quint, $J=7.5 \mathrm{~Hz}, 4 \mathrm{H}$ ), 1.61 (quint, $J=7.5 \mathrm{~Hz}, 4 \mathrm{H}$ ), 2.57 (t, $J=7.5 \mathrm{~Hz}, 4 \mathrm{H}$ ), $3.63(\mathrm{t}, J=7.5 \mathrm{~Hz}, 4 \mathrm{H}), 7.08(\mathrm{~s}, 4 \mathrm{H}) ;{ }^{13} \mathrm{C} \mathrm{NMR}\left(125 \mathrm{MHz}, \mathrm{CDCl}_{3}\right) \delta=25.6(2 \mathrm{C}), 29.0$ (2C), 31.5 (2C), 32.7 (2C), 35.4 (2C), 63.0 (2C), 128.2 (4C), 139.9 (2C); MS (EI): $\mathrm{m} / \mathrm{z}$ (\%): 278 (42) $\left[M^{+}\right], 117$ (100); high-resolution MS (EI, $70 \mathrm{eV}$ ): calc. for $\mathrm{C}_{18} \mathrm{H}_{30} \mathrm{O}_{2}$ : 278.2246, found: 278.2245 . 
Methyl 2,5-bis(5-hydroxypentyl)benzoate: quant. yield; colorless oil; ${ }^{1} \mathrm{H}$ NMR $\left(500 \mathrm{MHz}, \mathrm{CDCl}_{3}\right) \delta=$ $1.35-1.47(\mathrm{~m}, 4 \mathrm{H}), 1.53-1.70(\mathrm{~m}, 10 \mathrm{H}), 2.60(\mathrm{t}, J=7.7$

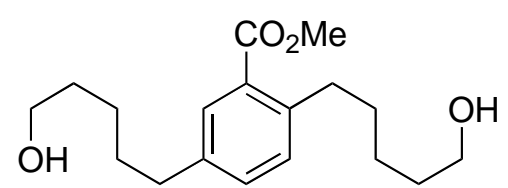
$\mathrm{Hz}, 2 \mathrm{H}), 2.90(\mathrm{t}, J=7.7 \mathrm{~Hz}, 2 \mathrm{H}), 3.63$ (ddd, $J=6.9,6.6$, $6.3 \mathrm{~Hz}, 4 \mathrm{H}), 3.88(\mathrm{~s}, 3 \mathrm{H}), 7.14(\mathrm{~d}, J=7.7 \mathrm{~Hz}, 1 \mathrm{H}), 7.23(\mathrm{dd}, J=7.7,2.0 \mathrm{~Hz}, 1 \mathrm{H}), 7.66$ $(\mathrm{d}, J=2.0 \mathrm{~Hz}, 1 \mathrm{H}) ;{ }^{13} \mathrm{C} \mathrm{NMR}\left(125 \mathrm{MHz}, \mathrm{CDCl}_{3}\right) \delta=25.3,25.7,30.1,31.5,32.5,34.0$, $35.2,51.8,62.8,62.9,129.2,130.4,130.9,132.0,140.0,141.7,168.4 ; \mathrm{MS}(\mathrm{EI}): \mathrm{m} / z$ (\%): 308 (21) $\left[M^{+}\right], 277$ (100); high-resolution MS (EI, $70 \mathrm{eV}$ ): calc. for $\mathrm{C}_{18} \mathrm{H}_{28} \mathrm{O}_{4}$ : 308.1988, found: 308.1990 .

\section{2-(tert-Butyldiphenylsilyloxy)methyl)-1,4-bis(5-(tetrahydro-2-2H-pyranyloxy)penty}

l)benzene. A solution of methyl 2,5-bis(5-hydroxypentyl)benzoate (1.0 g, $3.24 \mathrm{mmol}$ ), 3,4-dihydro- $2 \mathrm{H}$-pyran, $(0.74 \mathrm{ml}, 8.10 \mathrm{mmol})$ and in anhydrous dichloromethane $(35 \mathrm{ml})$ was stirred in the presence of a catalytic amount of $p$-toluenesulfonic acid ( $13 \mathrm{mg}$, $0.065 \mathrm{mmol}$ ) at room temperature for $14 \mathrm{~h}$. Water was added and the mixture was extracted with ethyl acetate and the organic layer was washed with brine. After removal of solvent in vacuo, the residue was chromatographed on silica gel with hexane-ethyl acetate $(5 / 1)$ to give the corresponding DHP ether in quantitative yield.

Methyl 2,5-bis(5-(tetrahydro-2-2H-pyranyloxy)pentyl)benzoate: colorless oil: ${ }^{1} \mathrm{H}$ NMR $(500 \mathrm{MHz}$, $\left.\mathrm{CDCl}_{3}\right) \delta=1.41-1.70(\mathrm{~m}, 24 \mathrm{H}), 2.61(\mathrm{t}, J=8.02 \mathrm{~Hz}$,

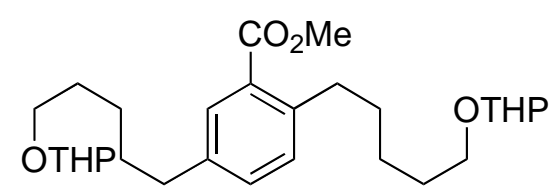
$2 \mathrm{H}), 2.91(\mathrm{t}, J=8.02 \mathrm{~Hz}, 2 \mathrm{H}), 3.36-3.40(\mathrm{~m}, 2 \mathrm{H})$, 3.47-3.50 (m, 2H), 3.71-3.76 (m, 2H), 3.88-3.84 (m, 5H), 4.56-4.58 (m, 2H), $7.14(\mathrm{~d}, J$ $=7.45 \mathrm{~Hz}, 1 \mathrm{H}), 7.22(\mathrm{dd}, J=7.45,1.71 \mathrm{~Hz}, 1 \mathrm{H}), 7.66(\mathrm{~d}, J=1.71,1 \mathrm{H}) ;{ }^{13} \mathrm{C} \mathrm{NMR}(125$ $\left.\mathrm{MHz} \mathrm{CDCl}_{3}\right) \delta=19.6,19.7,25.46$ 25.48, 25.9, 26.3, 29.1, 29.5, 29.6, 30.7, 31.2, 31.6, $34.0,35.2,51.8,62.25,62.32$, 67.4, 67.5, 98.78, 98.83, 129.2, 130.3, 130.8, 131.9, $140.1,141.7,168.3$ and one carbon signal is overlapped with the remaining signals; high-resolution MS (FAB+): calc. for $\mathrm{C}_{28} \mathrm{H}_{44} \mathrm{O}_{6} \mathrm{Na}$ : 499.3036, found: 499.3034 .

The product thus obtained was dissolved in ether $(20 \mathrm{ml})$ and was added dropwise slowly to a suspension of $\mathrm{LiAlH}_{4}(123 \mathrm{mg}, 3.24 \mathrm{mmol})$ in ether $(30 \mathrm{ml})$ and the reaction mixture was stirred at room temperature for $30 \mathrm{~min}$. The reaction was quenched with water $(0.15 \mathrm{ml})$ and $1 \mathrm{~N}-\mathrm{NaOH}$ aq $(0.10 \mathrm{ml})$ and the mixture was further stirred fot 20 min. After addition of $\mathrm{MgSO}_{4}$, the whole mixture was filtered and the filtrate was 
concentrated in vacuo to give the crude alcohol product in quantitative yield.

\section{2-Hydroxymethyl-1,4-bis(5-(tetrahydro-2-2H-pyr}

anyloxy)pentyl)benzene: colorless oil: ${ }^{1} \mathrm{H}$ NMR $\left(500 \mathrm{MHz}, \mathrm{CDCl}_{3}\right) \delta=1.41-1.72(\mathrm{~m}, 24 \mathrm{H}), 2.59(\mathrm{t}, J$ $=7.5 \mathrm{~Hz}, 2 \mathrm{H}), 2.65(\mathrm{t}, J=7.5 \mathrm{~Hz}, 2 \mathrm{H}), 3.35-3.41(\mathrm{~m}$,

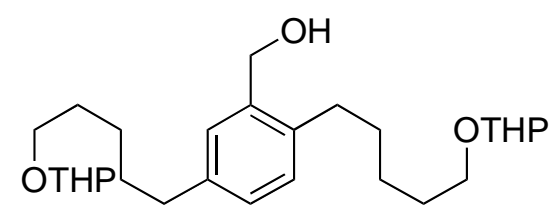
$2 \mathrm{H}), 3.47-3.50(\mathrm{~m}, 2 \mathrm{H}), 3.70-3.75(\mathrm{~m}, 2 \mathrm{H}), 3.83-3.87(\mathrm{~m}, 2 \mathrm{H}), 4.56(\mathrm{q}, J=4.0 \mathrm{~Hz}, 2 \mathrm{H})$, $4.69(\mathrm{~d}, J=4.0 \mathrm{~Hz}, 2 \mathrm{H}), 7.04(\mathrm{~d}, J=7.5 \mathrm{~Hz}, 1 \mathrm{H}), 7.09$ (d, $J=7.5 \mathrm{~Hz}, 1 \mathrm{H}), 7.18(\mathrm{~s}, 1 \mathrm{H})$; ${ }^{13} \mathrm{C}$ NMR (125 MHz, $\mathrm{CDCl}_{3}$ ) $\delta=19.6$ (2C), 25.5 (2C), 25.9, 26.3, 29.6 (2C), 30.7 (2C), 31.1, 31.3, 31.9, 35.4, 62.3 (2C), 63.1, 67.4, 67.5, 98.8 (2C), 127.8, 128.2, 129.3, 137.9, 138.0, 140.4; high-resolution MS (FAB+): calc. for $\mathrm{C}_{27} \mathrm{H}_{44} \mathrm{O}_{5} \mathrm{Na}$ : 471.3086, found: 471.3081 .

To the crude alcohol dissolved in DMF (30 ml) were added imidazole (441 mg, 6.48 $\mathrm{mmol})$ and TBDPSCl $(1.1 \mathrm{ml}, 4.21 \mathrm{mmol})$ and the reaction mixture was stirred at room temperature for $13 \mathrm{~h}$. After the reaction was complete, ether was added and the organic layer was washed with water, saturated aqueous sodium bicarbonate, and brine. After removal of solvent in vacuo, the residual crude silyl ether was dissolved in ethanol (30 $\mathrm{ml}$ ) and was stirred with pyridinium $p$-toluenesulfonate (PPTS) $(81 \mathrm{mg}, 0.324 \mathrm{mmol})$ at $55{ }^{\circ} \mathrm{C}$ for $24 \mathrm{~h}$. Triethylamine $(150 \mu \mathrm{l})$ was added and the mixture was concentrated in vacuo. Ether $(50 \mathrm{ml})$ was added and the ethereal layer was washed with water, $1 \mathrm{~N}$ hydrochloric acid, saturated aqueous sodium bicarbonate, and brine. After removal of solvent in vасио, the residue was purified by flash chromatography on silica gel with hexane-ethyl acetate (3/2) to give the desired diol (1.31 g, $78 \%$ in 3 steps).

\section{2-(tert-Butyldiphenylsilyloxymethyl)-1,4-bis(5-hy-}

droxypentyl)benzene. colorless oil; ${ }^{1} \mathrm{H}$ NMR $(500 \mathrm{MHz}$, $\left.\mathrm{CDCl}_{3}\right) \delta=1.08(\mathrm{~s}, 9 \mathrm{H}), 1.29-1.36(\mathrm{~m}, 2 \mathrm{H}), 1.38-1.44(\mathrm{~m}$, $2 \mathrm{H}), 1.47-1.68(\mathrm{~m}, 4 \mathrm{H}), 1.57-1.68(\mathrm{~m}, 4 \mathrm{H}), 2.47(\mathrm{t}, J=$

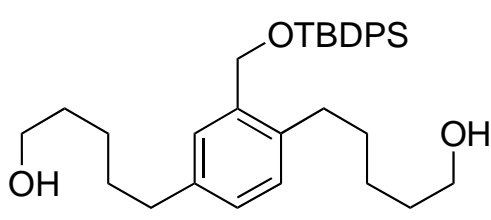
$7.5 \mathrm{~Hz}, 2 \mathrm{H}), 2.60(\mathrm{t}, J=7.5 \mathrm{~Hz}, 2 \mathrm{H}), 3.57(\mathrm{t}, J=6.5 \mathrm{~Hz}, 2 \mathrm{H}), 3.64(\mathrm{t}, J=6.5 \mathrm{~Hz}, 2 \mathrm{H})$, $4.74(\mathrm{~s}, 2 \mathrm{H}), 7.03(\mathrm{~m}, 2 \mathrm{H}), 7.31(\mathrm{~s}, 1 \mathrm{H}), 7.36-7.45(\mathrm{~m}, 6 \mathrm{H}), 7.69-7.71(\mathrm{~m}, 4 \mathrm{H}) ;{ }^{13} \mathrm{C}$ $\operatorname{NMR}\left(125 \mathrm{MHz}, \mathrm{CDCl}_{3}\right) \delta=19.3,25.4,25.7,26.8$ (3C), 30.5, 31.1, 31.7, 32.6, 32.7, $35.5,62.9,63.0,63.6,127.0,127.7,128.7,129.7,133.6,134.8,135.6,136.9$, 138.0, 139.9; high-resolution MS (FAB+): calc. for $\mathrm{C}_{33} \mathrm{H}_{46} \mathrm{O}_{3} \mathrm{SiNa}$ : 541.3114, found: 541.3115 . 
2-(Benzyloxymethyl)-1,4-bis(5-hydroxypentyl)benzene. To a solution of 2-hydroxymethyl-1,4-bis(5-(tetrahydro-2-2H-pyranyloxy)pentyl)benzene (200 mg, 0.446 $\mathrm{mmol})$ in dichloromethane $(3 \mathrm{ml})$ was added $60 \mathrm{wt} \%$ of $\mathrm{NaH}(38.6 \mathrm{mg}, 1.60 \mathrm{mmol})$ and benzyl bromide $(64.2 \mu \mathrm{l}, 0.535 \mathrm{mmol})$, successively. After the mixture was stirred at room temperature overnight, 15 -crown-5 ether ( $88.5 \mu 1,0.446 \mathrm{mmol})$ was added and the reaction mixture was further stirred at room temperatrure for $30 \mathrm{~min}$. Water was added, the aqueous layer was extracted with dichloromethane, and the combined organic layer was concentrated in vacuo to give the crude product which was purified by flash chromatography on silica gel with hexane-ethyl acetate (10/1) to afford 2-(benzyloxymethyl)-1,4-bis(5-(tetrahydro-2-2H-pyranyloxy)pentyl)benzene. To a solution of the product thus obtained in methanol $(5 \mathrm{ml})$ was added $1 \mathrm{~N}$ hydrochloric acid $(0.5 \mathrm{ml})$ and the mixture was stirred at $50{ }^{\circ} \mathrm{C}$ for $1 \mathrm{~h}$. The mixture was neutralized with $1 \mathrm{~N}$ aqueous sodium hydroxide and the aqueous layer was extracted with dichloromethane. The combined organic layer was concentrated in vacuo to give crude diol which was isolated by flash chromatography on silica gel with hexane-ethyl acetate (1/1) to give 2-(benzyloxymethyl)-1,4-bis(5-hydroxypentyl)benzene (151.3 $\mathrm{mg}, 91 \%)$ as a colorless oil: ${ }^{1} \mathrm{H}$ NMR $\left(500 \mathrm{MHz}, \mathrm{CDCl}_{3}\right) \delta=$ $1.36-1.43(\mathrm{~m}, 4 \mathrm{H}), 1.54-1.66(\mathrm{~m}, 8 \mathrm{H}), 2.58-2.62(\mathrm{~m}$, $4 \mathrm{H}), 3.59-3.64(\mathrm{~m}, 4 \mathrm{H}), 4.53(\mathrm{~s}, 2 \mathrm{H}), 4.57(\mathrm{~s}, 2 \mathrm{H}), 7.05$ $(\mathrm{d}, J=7.45 \mathrm{~Hz}, 1 \mathrm{H}), 7.09$ (d, $J=7.45 \mathrm{~Hz}, 1 \mathrm{H}), 7.17$ (s,

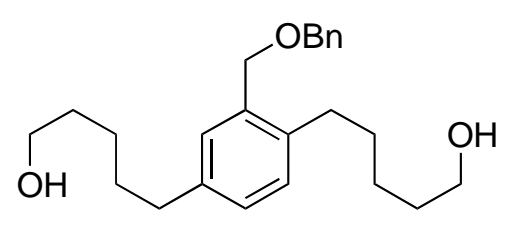
$1 \mathrm{H}), 7.28-7.38(\mathrm{~m}, 5 \mathrm{H}) ;{ }^{13} \mathrm{C} \mathrm{NMR}\left(125 \mathrm{MHz}, \mathrm{CDCl}_{3}\right) \delta=25.4,25.8,31.0,31.2,32.0$, $32.63,32.64,35.4,62.89,62.92,70.3,72.4,127.6,127.88$ (2C), 127.94, 128.4 (2C), 129.3, 129.4, 135.3, 138.3, 138.6, 140.0; high-resolution MS (EI, $70 \mathrm{eV}$ ): calc. for $\mathrm{C}_{24} \mathrm{H}_{34} \mathrm{NaO}_{3}$ : 393.2406, found: 393.2433.

Oxidation of diols. Method A: Representative procedure of PCC oxidation. A solution of 1,4-bis(5-hydroxypentyl)benzene (150 mg, $0.600 \mathrm{mmol})$ in dichloromethane (10 ml) was stirred in the presence of PCC (517 mg, $2.40 \mathrm{mmol})$ and molecular seave 4A (200 $\mathrm{mg}$ of powder) in argon atmosphere at room temperature for $2 \mathrm{~h}$. The mixture was separated by flash chromatography on silica gel with hexane-ethyl acetate (3/1) to give 1,4-bis(5-oxopentyl)benzene (2c) (126 mg, 85\%). The compounds $\mathbf{2 a}, \mathbf{2 e}$, and $\mathbf{2 h}$ were synthesized form the corresponding diols according to the similar procedure.

Method B: The aldehydes $\mathbf{2 b - d}$ and $\mathbf{2 f}$,g were prepared under the standard Swern 
oxidation conditions.

1,4-Bis(4-oxobutyl)benzene (2a): $78 \%$ yield; colorless oil; ${ }^{1} \mathrm{H}$ NMR $\left(500 \mathrm{MHz}, \mathrm{CDCl}_{3}\right) \delta=1.95$ (quint, $J=7.5$

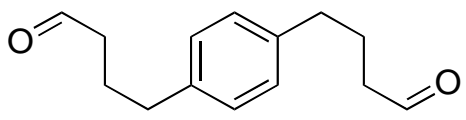
$\mathrm{Hz}, 4 \mathrm{H}), 2.45(\mathrm{td}, J=7.5,1.5 \mathrm{~Hz}, 4 \mathrm{H}), 2.63(\mathrm{t}, J=7.5 \mathrm{~Hz}$, $4 \mathrm{H}), 7.10(\mathrm{~s}, 4 \mathrm{H}), 9.75(\mathrm{t}, J=1.5 \mathrm{~Hz}, 2 \mathrm{H}) ;{ }^{13} \mathrm{C} \mathrm{NMR}\left(125 \mathrm{MHz}, \mathrm{CDCl}_{3}\right) \delta=23.6(2 \mathrm{C})$, 34.5 (2C), 43.1 (2C), 128.5 (4C), 138.9 (2C), 202.3 (2C); high-resolution MS (ESI-): calc. for $\mathrm{C}_{14} \mathrm{H}_{17} \mathrm{O}_{2}: 217.1229$, found: 217.1222 .

5-(4-(4-Oxobutyl)phenyl)pentanal (2b): $79 \%$ yield; colorless oil; ${ }^{1} \mathrm{H}$ NMR $\left(500 \mathrm{MHz}, \mathrm{CDCl}_{3}\right) \delta=1.66(\mathrm{~m}$, $4 \mathrm{H}$ ), 1.94 (quint, $J=7.5 \mathrm{~Hz}, 2 \mathrm{H}), 2.44(\mathrm{td}, J=7.5,1.5 \mathrm{~Hz}$,

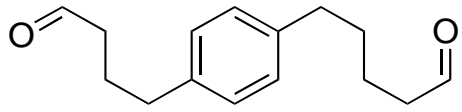
4H), $2.61(\mathrm{~m}, 4 \mathrm{H}), 7.08(\mathrm{~m}, 4 \mathrm{H}), 9.75(\mathrm{t}, J=1.5 \mathrm{~Hz}, 2 \mathrm{H}) ;{ }^{13} \mathrm{C} \mathrm{NMR}\left(125 \mathrm{MHz}, \mathrm{CDCl}_{3}\right)$ $\delta=21.6,23.6,30.8,34.5,35.1,43.1,43.6,128.3$ (2C), $128.4(2 \mathrm{C}), 138.6,139.6,202.3$, 202.4; high-resolution MS (ESI-): calc. for $\mathrm{C}_{15} \mathrm{H}_{19} \mathrm{O}_{2}$ : 231.1385, found: 231.1381 .

1,4-Bis(5-oxopentyl)benzene (2c): $92 \%$ yield; colorless oil; ${ }^{1} \mathrm{H}$ NMR $\left(400 \mathrm{MHz}, \mathrm{CDCl}_{3}\right) \delta=1.57-1.75(\mathrm{~m}, 8 \mathrm{H})$,

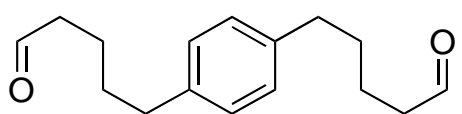
$2.45(\mathrm{td}, J=7.3,1.5 \mathrm{~Hz}, 4 \mathrm{H}), 2.60(\mathrm{t}, J=7.3,4 \mathrm{H}), 7.08(\mathrm{~s}$, $4 \mathrm{H}), 9.76(\mathrm{t}, J=1.5,2 \mathrm{H}) ;{ }^{13} \mathrm{C}$ NMR $\left(100 \mathrm{MHz}, \mathrm{CDCl}_{3}\right) \delta=21.5(2 \mathrm{C}), 30.8(2 \mathrm{C}), 35.0$ (2C), 43.6 (2C), 128.2 (4C), 139.2 (2C), 202.5 (2C); high-resolution MS (EI, $70 \mathrm{eV}$ ): calc. for $\mathrm{C}_{16} \mathrm{H}_{22} \mathrm{O}_{2}$ : 246.1620, found: 246.1617 .

6-(4-(5-Oxopentyl)phenyl)hexanal (2d): $95 \%$ yield; colorless oil; ${ }^{1} \mathrm{H} \quad \mathrm{NMR} \quad\left(500 \mathrm{MHz}, \mathrm{CDCl}_{3}\right) \quad \delta$ $=1.35-1.37(\mathrm{~m}, 2 \mathrm{H}), 1.60-1.69(\mathrm{~m}, 8 \mathrm{H}), 2.40-2.45(\mathrm{~m}$,

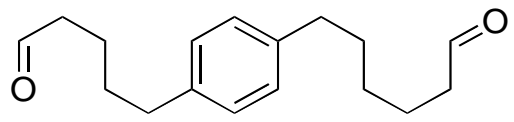
$4 \mathrm{H}), 2.45-2.60(\mathrm{~m}, 4 \mathrm{H}), 7.08(\mathrm{~s}, 4 \mathrm{H}), 9.75(\mathrm{~s}, 2 \mathrm{H}) ;{ }^{13} \mathrm{C} \mathrm{NMR}\left(125 \mathrm{MHz}, \mathrm{CDCl}_{3}\right) \delta=$ 21.6, 21.9, 28.7, 30.9, 31.2, 35.1, 35.2, 43.7, 43.8, 128.2 (2C), 128.2 (2C), 128.3, 139.2, 202.6, 202.7; high-resolution MS (ESI-): calc. for $\mathrm{C}_{17} \mathrm{H}_{23} \mathrm{O}_{2}$ : 259.1698, found: 259.1686 .

1,4-Bis(6-oxohexyl)benzene (2e): $81 \%$ yield; colorless oil; ${ }^{1} \mathrm{H}$ NMR $\left(400 \mathrm{MHz}, \mathrm{CDCl}_{3}\right) \delta=$ $1.28-1.40(\mathrm{~m}, 4 \mathrm{H}), 1.59-1.68(\mathrm{~m}, 8 \mathrm{H}), 2.41(\mathrm{td}, J=$

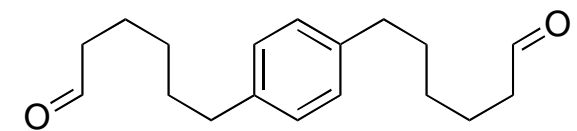
6.0, $1.2 \mathrm{~Hz}, 4 \mathrm{H}), 2.57(\mathrm{t}, J=6.0 \mathrm{~Hz}, 4 \mathrm{H}), 7.06(\mathrm{~s}, 4 \mathrm{H}), 9.75(\mathrm{t}, J=1.5 \mathrm{~Hz}, 2 \mathrm{H}) ;{ }^{13} \mathrm{C}$ NMR (100 MHz, $\left.\mathrm{CDCl}_{3}\right) \delta=21.8$ (2C), 28.7 (2C), 31.1 (2C), 35.2 (2C), $43.7(2 \mathrm{C})$, 128.2 (4C), 139.6 (2C), 202.7 (2C); high-resolution MS (ESI-): calc. for $\mathrm{C}_{18} \mathrm{H}_{25} \mathrm{O}_{2}$ : 273.1855 , found: 273.1847 . 
2-(tert-Butyldiphenylsilyloxymethyl)-1,4-bis(5-oxopen-

tyl)benzene (2f): $98 \%$ yield; colorless oil; ${ }^{1} \mathrm{H}$ NMR (500 $\left.\mathrm{MHz}, \mathrm{CDCl}_{3}\right) \delta=1.08(\mathrm{~s}, 9 \mathrm{H}), 1.49-1.67(\mathrm{~m}, 8 \mathrm{H}), 2.34(\mathrm{t}$, $J=7.0 \mathrm{~Hz}, 2 \mathrm{H}), 2.44(\mathrm{t}, J=7.0 \mathrm{~Hz}, 2 \mathrm{H}), 2.49(\mathrm{t}, J=7.0$

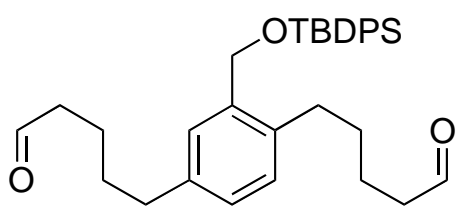
$\mathrm{Hz}, 2 \mathrm{H}), 2.61(\mathrm{t}, J=7.0 \mathrm{~Hz}, 2 \mathrm{H}), 4.73(\mathrm{~s}, 2 \mathrm{H}), 7.00-7.04(\mathrm{~m}, 2 \mathrm{H}), 7.26(\mathrm{~s}, 1 \mathrm{H})$, 7.36-7.45 (m, 6H), $7.69(\mathrm{~d}, J=8.02,4 \mathrm{H}), 9.69(\mathrm{~d}, J=1.72,1 \mathrm{H}), 9.75(\mathrm{~d}, J=1.72 \mathrm{~Hz}$, $1 \mathrm{H}) ;{ }^{13} \mathrm{C} \mathrm{NMR}\left(125 \mathrm{MHz}, \mathrm{CDCl}_{3}\right) \delta=19.3,21.7,22.0,26.8(3 \mathrm{C}), 30.1,30.8,31.4,35.3$, $43.7,43.8,63.7,127.1,127.5,127.7,128.8,129.7$, 133.5, 135.6, 136.6, 139.4, 143.2, 202.4, 202.5 and one carbon signal is hidden behind the other peaks; high-resolution MS (FAB+): calc. for $\mathrm{C}_{33} \mathrm{H}_{42} \mathrm{O}_{3} \mathrm{SiNa}$ : 537.2801, found: 537.2812 .

2-(Benzyloxymethyl)-1,4-bis(5-oxopentyl)benzene (2g). $77 \%$ yield; colorless oil; ${ }^{1} \mathrm{H} \mathrm{NMR}\left(500 \mathrm{MHz}, \mathrm{CDCl}_{3}\right) \delta=$ $1.56-1.68(\mathrm{~m}, 8 \mathrm{H}), 2.39(\mathrm{td}, J=7.3,1.5 \mathrm{~Hz}, 2 \mathrm{H}), 2.45(\mathrm{td}$, $J=7.3,1.5 \mathrm{~Hz}, 2 \mathrm{H}), 22.59-2.63(\mathrm{~m}, 4 \mathrm{H}), 4.52(\mathrm{~s}, 2 \mathrm{H})$,

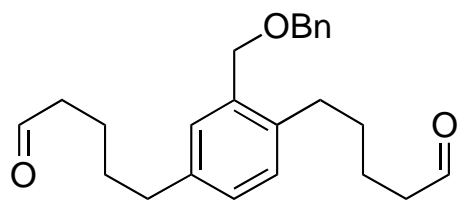
$4.57(\mathrm{~s}, 2 \mathrm{H}), 7.04(\mathrm{dd}, J=7.5,1.3 \mathrm{~Hz}, 1 \mathrm{H}), 7.08(\mathrm{~d}, J=7.5 \mathrm{~Hz}, 1 \mathrm{H}), 7.16(\mathrm{~d}, J=1.3 \mathrm{~Hz}$, $1 \mathrm{H}), 7.27-7.38(\mathrm{~m}, 5 \mathrm{H}), 9.71(\mathrm{t}, J=1.5 \mathrm{~Hz}, 1 \mathrm{H}), 9.76(\mathrm{t}, J=1.5 \mathrm{~Hz}, 1 \mathrm{H}) ;{ }^{13} \mathrm{C} \mathrm{NMR}$ $\left(125 \mathrm{MHz}, \mathrm{CDCl}_{3}\right) \delta=21.7,22.0,30.7,30.8,30.9,31.7,35.1,43.7,70.3,72.4,127.7$ (2C), 127.9, 128.0, 128.4 (2C), 129.3, 129.5, 135.4, 138.2, 138.3, 139.5, 202.5, 202.5; high-resolution MS (ESI-): calc. for $\mathrm{C}_{24} \mathrm{H}_{29} \mathrm{O}_{3}$ : 365.2117, found: 365.2098.

6-(4-(4-Oxobutyl)phenyl)hexanal (2h): $72 \%$ yield; colorless oil; ${ }^{1} \mathrm{H} \quad \mathrm{NMR} \quad\left(400 \mathrm{MHz}, \mathrm{CDCl}_{3}\right) \quad \delta$ $=1.34-1.40(\mathrm{~m}, 2 \mathrm{H}), 1.62-1.67(\mathrm{~m}, 4 \mathrm{H}), 1.95$ (quint, $\mathrm{J}$

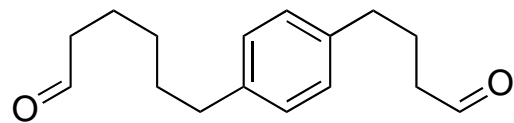
$=7.5 \mathrm{~Hz}, 2 \mathrm{H}), 2.42-2.45(\mathrm{~m}, 4 \mathrm{H}), 2.56-2.64(\mathrm{~m}, 4 \mathrm{H}), 7.09(\mathrm{~s}, 4 \mathrm{H}), 9.74-9.77(\mathrm{~m}, 2 \mathrm{H})$; ${ }^{13} \mathrm{C}$ NMR $\left(100 \mathrm{MHz}, \mathrm{CDCl}_{3}\right) \delta=21.9,23.7,28.7,31.2,34.5,35.2,43.1,43.8,128.3$ (2C), 128.4 (2C), 138.5, 140.0, 202.4, 202.7; high-resolution MS (ESI-): calc. for $\mathrm{C}_{16} \mathrm{H}_{21} \mathrm{O}_{2}: 245.1542$, found: 245.1536 .

Intramolecular pinacol coupling reactions for synthesis of 3a-h. Representative Procedure. To a $0.1 \mathrm{M}$ solution of samarium(II) iodide in THF (200 ml, $20 \mathrm{mmol}$ ) was added magnesium powder (24.3 g, $1.00 \mathrm{~mol})$, HMPA $(7.0 \mathrm{ml}, 40 \mathrm{mmol})$, and chlorotrimethylsilane $(8.3 \mathrm{ml}, 65 \mathrm{mmol})$ at room temperature in argon atmosphere. A solution of 1,4-bis(5-oxopentyl)benzene (2c) (8.5 g, $34.5 \mathrm{mmol})$ and TMSCl (16.7 ml, $131 \mathrm{mmol})$ in deoxygenated THF (100 ml) was added dropwise during $72 \mathrm{~h}$ and the 
whole reaction mixture was stirred at room temperature for further $12 \mathrm{~h}$. The magnesium powder was removed off and the mixture was diluted with ether $(200 \mathrm{ml})$. The ethereal layer was washed with $1 \mathrm{~N}$ hydrochloric acid $(3 \times 100 \mathrm{ml})$ and the aqueous layer was extracted with ether $(100 \mathrm{ml})$. The combined ethereal layer was further washed with brine and was dried over $\mathrm{MgSO}_{4}$. After removal of solvent in vacuo, the residue was separated by flash chromatography on silica gel with hexane-ethyl acetate (2/1) to give [10]paracyclophanediol 3c $(5.5 \mathrm{~g}, 64 \%)$ as a threo/erythro mixture (ca. 1/1). The other cyclophanediols 3a,b and $\mathbf{3 d} \mathbf{d} \mathbf{h}$ were prepared form the corresponding $\mathbf{2 a}, \mathbf{b}$ and $\mathbf{2 d - h}$, respectively, according to the similar procedure. Yields are listed in Table 2. [8]Paracyclophanediol 3a: white solid; m.p. 71.5-72.0 ${ }^{\circ} \mathrm{C} ;{ }^{1} \mathrm{H}$ NMR $\left(500 \mathrm{MHz}, \mathrm{CDCl}_{3}\right) \delta=1.04(\mathrm{ddd}, J=13.7,9.7,2.3 \mathrm{~Hz}, 2 \mathrm{H}), 1.29-1.35$ $(\mathrm{m}, 2 \mathrm{H}), 1.49-1.58(\mathrm{~m}, 2 \mathrm{H}), 1.76-1.83(\mathrm{~m}, 2 \mathrm{H}), 1.87-1.89(\mathrm{~m}, 2 \mathrm{H})$, 2.39-2.45 (m, 2H), 2.95 (dt, $J=12.6,4.6 \mathrm{~Hz}, 2 \mathrm{H}), 7.06(\mathrm{dd}, J=7.4,1.7$ $\mathrm{Hz}, 2 \mathrm{H}), 7.21(\mathrm{dd}, J=7.4,1.7 \mathrm{~Hz}, 2 \mathrm{H}) ;{ }^{13} \mathrm{C} \mathrm{NMR}\left(125 \mathrm{MHz}, \mathrm{CDCl}_{3}\right) \delta$

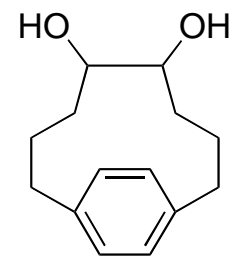
$=29.1,34.8,35.4,74.9,129.6,130.5,140.5$; high-resolution MS (EI, $70 \mathrm{eV})$ : calc. for $\mathrm{C}_{14} \mathrm{H}_{20} \mathrm{O}_{2}: 220.1463$, found: 220.1467 .

[9]Paracyclophanediol 3b: white solid; m.p. 146.7-148.1 ${ }^{\circ} \mathrm{C} ;{ }^{1} \mathrm{H}$ NMR $\left(500 \mathrm{MHz}, \mathrm{CDCl}_{3}\right) \delta=-0.94-0.75(\mathrm{~m}, 2 \mathrm{H}), 0.88-2.43(\mathrm{~m}, 8 \mathrm{H})$, 2.43-2.72 (m, 4H), 2.90-3.26 (m, 2H), 7.04-7.17 (m, 4H); ${ }^{13} \mathrm{C}$ NMR $\left(125 \mathrm{MHz}, \mathrm{CDCl}_{3}\right) \delta=21.3,23.1,24.6,25.6,26.8,27.0,27.9,29.7$,

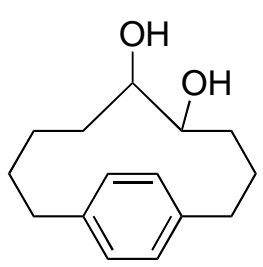
$29.7,32.3,34.3,34.8,35.3,35.6,73.1,73.7,73.8,74.8,128.3,128.6$, 129.4, 129.5, 129.7, 129.9, 130.8, 130.8, 139.3, 140.0, 140.0, 140.1; high-resolution MS (EI, $70 \mathrm{eV}$ ): calc. for $\mathrm{C}_{15} \mathrm{H}_{22} \mathrm{O}_{2}: 234.1620$, found: 234.1619 .

[10]Paracyclophanediol 3c: white solid; m.p. 65.5-67.8 ${ }^{\circ} \mathrm{C} ;{ }^{1} \mathrm{H}$ NMR $\left(400 \mathrm{MHz}, \mathrm{CDCl}_{3}\right) \delta=0.17(\mathrm{~m}, 1 \mathrm{H}), 0.49$ (dddd, $J=14.4,11.6$ 4.8, $3.6 \mathrm{~Hz}, 1 \mathrm{H}), 0.72(\mathrm{~m}, 1 \mathrm{H}), 0.87-0.94(\mathrm{~m}, 2 \mathrm{H}), 1.11(\mathrm{~m}, 1 \mathrm{H})$, 1.30-1.47 (m, 3H), 1.52-1.74 (m, 3H), 1.87 (brs, $1 \mathrm{H}), 1.92(\mathrm{~m}, 1 \mathrm{H})$,

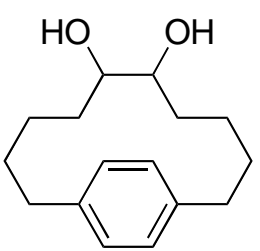
2.51 (ddd, $J=13.2,10.4,5.2 \mathrm{~Hz}, 1 \mathrm{H}), 2.60$ (ddd, $J=13.2,8.8,5.2$ $\mathrm{Hz}, 1 \mathrm{H}$ ), 2.75 (ddd, $J=13.2,5.6,5.2 \mathrm{~Hz}, 1 \mathrm{H}$ ), 2.78 (br m, 1H), 2.85 (dt, $J=13.2,5.2$ $\mathrm{Hz}, 1 \mathrm{H}), 3.22$ (br m, 1H), 7.04-7.19 (m, 4H); $\left.{ }^{13} \mathrm{C} \mathrm{NMR} \mathrm{(100} \mathrm{MHz,} \mathrm{CDCl}_{3}\right) \delta=22.1$, 22.2, 28.3, 28.5, 29.7, 31.0, 35.0, 35.1, 73.9, 74.7, 129.2, 129.3, 129.6, 129.7, 138.9, 139.5; MS (EI): $\mathrm{m} / z(\%): 248(54)\left[\mathrm{M}^{+}\right], 145$ (100); high-resolution MS (EI, $\left.70 \mathrm{eV}\right)$ : calc. for $\mathrm{C}_{16} \mathrm{H}_{24} \mathrm{O}_{2}$ : 248.1776, found: 248.1780. 
[11]Paracyclophanediol 3d: colorless syrup; ${ }^{1} \mathrm{H}$ NMR $(400 \mathrm{MHz}$, $\left.\mathrm{CDCl}_{3}\right) \delta=0.39-0.69(\mathrm{~m}, 1 \mathrm{H}), 0.81-1.90(\mathrm{~m}, 13 \mathrm{H}), 2.10$ (brs, 2H), 2.44- 2.89 (m, 4H), 3.12 (dt, $J=10.4,3.2 \mathrm{~Hz}, 0.5 \mathrm{H}), 3.28-3.32$ (m, $0.5 \mathrm{H}), 3.57-3.66(\mathrm{~m}, 0.5 \mathrm{H}), 3.77-3.80(\mathrm{~m}, 0.5 \mathrm{H}), 7.05-7.16(\mathrm{~m}, 4 \mathrm{H})$; ${ }^{13} \mathrm{C}$ NMR $\left(100 \mathrm{MHz}, \mathrm{CDCl}_{3}\right) \delta=23.66,23.71,25.0,27.1,28.41$,

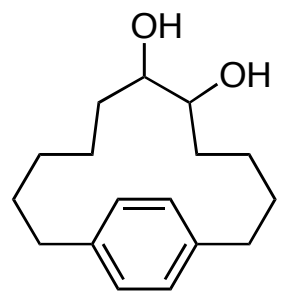
28.44, 28.76, 28.82, 28.9, 29.5, 29.7 31.6, 32.3, 32.6, 36.0, 36.1, 36.4, 36.5, 73.4, 73.5, $73.7,74.3,128.9,129.0,129.0,129.0,129.2,129.3,140.0,140.1,140.4,140.4$ and two carbon signals are overlapped with some of the other signals; high-resolution MS (EI, $70 \mathrm{eV}$ ): calc. for $\mathrm{C}_{17} \mathrm{H}_{26} \mathrm{O}_{2}: 262.1933$, found: 262.1940 .

[12]Paracyclophanediol 3e: white solid; m.p. 108.4-110.1 ${ }^{\circ} \mathrm{C} ;{ }^{1} \mathrm{H}$ $\operatorname{NMR}\left(500 \mathrm{MHz}, \mathrm{CDCl}_{3}\right) \delta=0.82-0.89(\mathrm{~m}, 2 \mathrm{H}), 1.01-1.02(\mathrm{~m}, 2 \mathrm{H})$, 1.14-1.35 (m, 6H), 1.48-1.61 (m, 4H), 1.73-1.78 (m, 2H), 2.53 (ddd, $J=13.0,9.5,3.0 \mathrm{~Hz}, 1 \mathrm{H}), 2.57(\mathrm{dd}, J=8.0,3.5 \mathrm{~Hz}, 1 \mathrm{H}), 2.64$ (ddd, $J=13.0,9.5,3.5 \mathrm{~Hz}, 1 \mathrm{H}), 2.72(\mathrm{ddd}, J=13.0,7.5,3.5 \mathrm{~Hz}, 1 \mathrm{H}), 2.97$

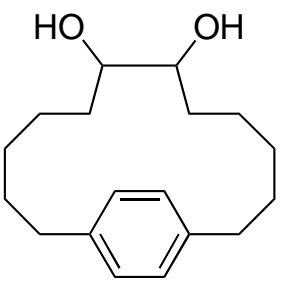
$(\mathrm{q}, 6.0 \mathrm{~Hz}, 1 \mathrm{H}), 3.22(\mathrm{~m}, 1 \mathrm{H}), 7.11(\mathrm{~s}, 4 \mathrm{H}) ;{ }^{13} \mathrm{C} \mathrm{NMR}\left(125 \mathrm{MHz}, \mathrm{CDCl}_{3}\right) \delta=24.3,24.4$, 26.3, 27.1, 29.5, 29.7, 29.9, 32.5, 35.2, 35.5, 70.6, 73.8, 128.3, 128.8, 140.1 and one carbon signal is hidden behind the remaining signals; high-resolution MS (EI, $70 \mathrm{eV})$ : calc. for $\mathrm{C}_{18} \mathrm{H}_{28} \mathrm{O}_{2}$ : 276.2089, found: 276.2095 .

[10]Paracyclophanediol 3f: colorless oil; ${ }^{1} \mathrm{H}$ NMR $(500 \mathrm{MHz}$, $\left.\mathrm{CDCl}_{3}\right) \delta=-0.2-0.56(\mathrm{~m}, 2 \mathrm{H}), 0.61-0.78(\mathrm{~m}, 1 \mathrm{H}), 0.79-1.04$ $(\mathrm{m}, 3 \mathrm{H}) .1 .01-1.15(\mathrm{~m}, 10 \mathrm{H}), 1.16-1.86(\mathrm{~m}, 8 \mathrm{H}), 2.04$ (brs, $2 \mathrm{H})$, 2.30-2.93 (m, 5H), 3.11-3.25 (m, $1 \mathrm{H}), 4.72-4.88(\mathrm{~m}, 2 \mathrm{H})$, 6.94-7.08 (m, 2H), 7.33-7.45 (m, 7H), $7.71(\mathrm{~d}, J=7.0 \mathrm{~Hz}, 4 \mathrm{H})$;

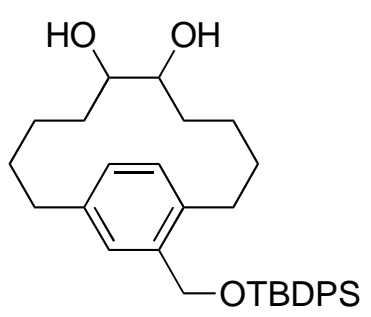
${ }^{13} \mathrm{C}$ NMR $\left(125 \mathrm{MHz}, \mathrm{CDCl}_{3}\right) \delta=14.2,19.3,20.8,21.0,21.0,21.7,21.8,22.3,23.1$, $23.3,24.8,25.9,26.6,26.7,26.8,26.9,28.0,28.0,28.2,28.7,30.2,30.4,30.5,30.8$, $31.7,32.0,32.2,32.2,33.0,33.2,33.2,33.3,34.9,35.0,35.2,35.7,60.4,63.1,63.3$, 63.6, 73.8, 73.8, 73.9, 74.0, 74.3, 74.6, 74.7, 74.8, 127.6, 127.6, 127.7, 127.7, 127.8, 128.1, 128.1, 128.3, 128.4, 128.5, 129.6, 129.7, 129.7, 129.9, 130.2, 130.3, 130.9, 133.4, 133.5, 133.5, 133.5, 135.5, 135.5, 135.6, 135.6, 136.0, 136.3, 138.9, 139.0, 139.1, 139.2, 139.5, 139.8; high-resolution MS (FAB+): calc. for $\mathrm{C}_{33} \mathrm{H}_{44} \mathrm{O}_{3} \mathrm{SiNa}$ : 539.2957, found: 539.2950 .

[10]Paracyclophanediol 3g: colorless oil; ${ }^{1} \mathrm{H}$ NMR (500 MHz, $\left.\mathrm{CDCl}_{3}\right) \delta-0.87-0.59$ $(\mathrm{m}, 1.5 \mathrm{H}), 0.60-1.98(\mathrm{~m}, 14 \mathrm{H}), 2.30-3.20(\mathrm{~m}, 4 \mathrm{H}), 3.47-3.59(\mathrm{~m}, 0.5 \mathrm{H}), 4.09-4.20(\mathrm{~m}$, 
$1 \mathrm{H})$, 4.36-4.64 (m, 3H), 7.00-7.37 (m, 7H), 7.43-7.48 (m, 0.5H), 7.61-7.66 (m, 0.5H); ${ }^{13} \mathrm{C}$ NMR $\left(125 \mathrm{MHz}, \mathrm{CDCl}_{3}\right) \delta=10.9,14.0$, $21.2,22.4,22.96,22.99,23.7,26.6,27.2,28.1,28.2,28.4,28.7$, $28.9,30.3,30.7,31.9,32.4,33.2,34.7,34.89,34.91,35.4,38.7$, $68.1,69.8,70.0,70.2,72.36,72.39,74.1,74.4,127.6,127.7,127.8$,

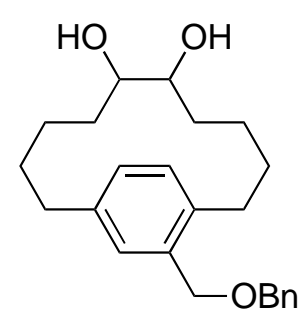
$127.88,127.92$, 128.4, 128.5, 128.8, 129.1, 129.3, 129.7, 129.9, 130.3, 130.6, 130.9, 132.4, 136.5, 137.7, 138.2, 138.3, 139.4, 167.7; high-resolution MS (FAB+): calc. for $\mathrm{C}_{24} \mathrm{H}_{32} \mathrm{O}_{3} \mathrm{Na}$ : 391.2249, found: 391.2250.

[10]Paracyclophanediol 3h: colorless oil; ${ }^{1} \mathrm{H}$ NMR $(400 \mathrm{MHz}$, $\left.\mathrm{CDCl}_{3}\right) \delta=-0.56-0.48(\mathrm{~m}, 0.5 \mathrm{H}), 0.08-0.19(\mathrm{~m}, 0.5 \mathrm{H}), 0.54-0.76(\mathrm{~m}$, $1 \mathrm{H})$, 0.84-1.47 (m, 5H), 1.50-1.73 (m, 2H), 1.92-2.03 (m, 3H), 2.14-2.35 (m, 0.5 H), 2.2-2.77 (m, 3H), 2.77-2.97 (m, 2H), 3.24-3.30

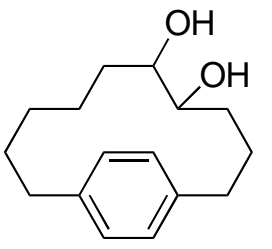
$(\mathrm{m}, 0.5 \mathrm{H}), 6.99-7.19(\mathrm{~m}, 4 \mathrm{H}) ;{ }^{13} \mathrm{C} \mathrm{NMR}\left(100 \mathrm{MHz}, \mathrm{CDCl}_{3}\right) \delta=$ 23.92, 23.95, 25.6, 26.1, 26.2, 26.6, 27.4, 28.8, 29.4, 29.8, 32.66, 32.69, 34.1, 34.7, 35.4, 35.9, 73.2, 73.8, 74.9, 75.2, 128.6, 129.0, 129.1, 129.4, 129.6, 129.67, 129.70, 130.7, 139.1, 139.3, 139.8, 139.9; high-resolution MS (EI, $70 \mathrm{eV}$ ): calc. for $\mathrm{C}_{16} \mathrm{H}_{24} \mathrm{O}_{2}$ : 248.1176, found: 248.1779 .

Ring Closure Metathesis for 4. To a solution of methyltriphenylphosphonium bromide $(583 \mathrm{mg}, 1.63 \mathrm{mmol})$ in THF $(8.0 \mathrm{ml})$ was added dropwise by a syringe at $-78{ }^{\circ} \mathrm{C} 1.6 \mathrm{M}$ $n$-butyllithium in hexane $(1.0 \mathrm{ml}, 1.61 \mathrm{mmol})$ and the mixture was stirred for $30 \mathrm{~min}$ and was further stirred at room temperature for $30 \mathrm{~min}$. After cooling down to $-78{ }^{\circ} \mathrm{C}$, a solution of aldehyde $2 \mathbf{c}(64 \mathrm{mg}, 0.26 \mathrm{mmol})$ in THF $(1.0 \mathrm{ml})$ was added slowly by a syringe and was stirred at $-78{ }^{\circ} \mathrm{C}$ for $15 \mathrm{~min}$ and, then, the reaction mixture was warmed to room temperature. After stirring for $1 \mathrm{~h}$, saturated $\mathrm{NH}_{4} \mathrm{Cl}$ aq was added and the aqueous layer was extracted with ether. The combined ethereal layer was washed with brine and was concentrated in vacuo, and was separated by flash chromatography on silica gel with hexane to give 1,4-di(5-hexenyl)benzene (60 mg, 95\%): colorless oil; ${ }^{1} \mathrm{H}$ $\operatorname{NMR}\left(500 \mathrm{MHz}, \mathrm{CDCl}_{3}\right) \delta=1.36$ (quint, $J=7.5 \mathrm{~Hz}, 4 \mathrm{H}$ ), 1.54 (quint, $J=7.5 \mathrm{~Hz}, 4 \mathrm{H}), 2.00(\mathrm{q}, J=7.5 \mathrm{~Hz}, 4 \mathrm{H})$, $2.50(\mathrm{t}, J=7.5 \mathrm{~Hz}, 4 \mathrm{H}), 4.85-4.94(\mathrm{~m}, 4 \mathrm{H}), 5.69-5.77(\mathrm{~m}$,

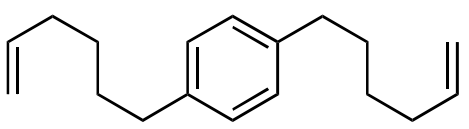
2H), 7.01 (s, 4H); ${ }^{13} \mathrm{C}$ NMR (125 MHz, $\left.\mathrm{CDCl}_{3}\right) \delta=28.6,31.0,33.7,35.4,114.3,128.2$, 138.9, 139.9; MS (EI): m/z (\%): 242 (72) $\left[\mathrm{M}^{+}\right], 173$ (100); high-resolution MS (EI, 70 
$\mathrm{eV}$ ): calc. for $\mathrm{C}_{18} \mathrm{H}_{26}: 242.2035$, found: 242.2007. The diene thus obtained (40 mg, $0.165 \mathrm{mmol})$ was dissolved in dichloromethane $(250 \mathrm{ml})$ and was heated at $50{ }^{\circ} \mathrm{C}$. A solution the first generation Grubbs catalyst (40 mg, $29 \mathrm{~mol} \%$ ) in dichloromethane (40 $\mathrm{ml}$ ) was added dropwise slowly and the reaction mixture was stirred at $50{ }^{\circ} \mathrm{C}$ for $14 \mathrm{~h}$. After cooling down to room temperature, Florisil (1.5 g) was added and the mixture was stirred for $30 \mathrm{~min}$ and was filtered through Celite. After removal of solvent in vacuo, the residue was chromatographed on Silica gel with hexane to give cyclic diene 4 (27 $\mathrm{mg}, 76 \%$ ) as a cis/trans mixture.

Cyclic diene 4: colorless oil; ${ }^{1} \mathrm{H}$ NMR $(500 \mathrm{MHz}$, $\left.\mathrm{CDCl}_{3}\right) \delta=1.29-1.50(\mathrm{~m}, 8 \mathrm{H}), 1.50-1.70(\mathrm{~m}, 8 \mathrm{H})$, $1.91-2.06(\mathrm{~m}, 8 \mathrm{H}), 2.54(\mathrm{t}, J=7.8 \mathrm{~Hz}, 8 \mathrm{H}), 5.32(\mathrm{t}, J=$ $3.9 \mathrm{~Hz}, 4 \mathrm{H}), 7.01-7.02(\mathrm{~m}, 8) ;{ }^{13} \mathrm{C}$ NMR $(125 \mathrm{MHz}$, $\left.\mathrm{CDCl}_{3}\right) \delta=26.8,28.6,28.6,29.0,30.2,30.2,30.3$,

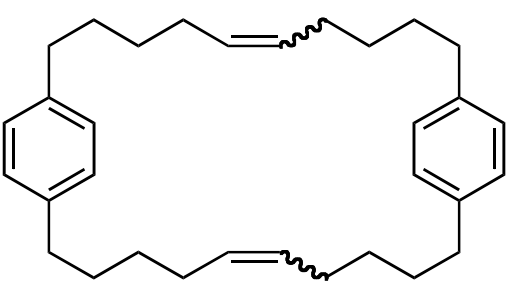
30.6, 32.0, 35.3, 35.4, 128.2, 128.2, 128.2, 130.1, 130.6, 139.8, 139.9; high-resolution MS (EI, $70 \mathrm{eV}$ ): calc. for $\mathrm{C}_{32} \mathrm{H}_{44}: 428.3443$, found: 428.3423 .

Reduction of 3c,d to dehydro[10]- and dehydro[11]-paracyclophanes. Representative Procedure. [11]Paracyclophanediol 3d (400 mg, $1.52 \mathrm{mmol}$ ) was dissolved in toluene $(20 \mathrm{ml})$ and the solution was stirred with imidazole $(724 \mathrm{mg}, 10.6 \mathrm{mmol})$ and chlorodiphenylphophine $(1.12 \mathrm{ml}, 6.09 \mathrm{mmol})$ at $80{ }^{\circ} \mathrm{C}$ for $10 \mathrm{~min}$. Iodine $(1.55 \mathrm{~g}, 6.09$ mmol) was added slowly and the reaction mixture was stirred under reflux for $1.5 \mathrm{~h}$. Ether was added and the mixture was washed with saturated aqueous sodium thiosulfate and $1 \mathrm{~N}$ aqueous sodium hydroxide. After removal of solvent in vacuo, the residue was chromatographed on silica gel with hexane to give the corresponding dehydro[11]paracyclophane (283 mg, 82\%) as a cis/trans mixture (ca. 1/1). Dehydro[10]paracyclophane was prepared form $3 \mathbf{c}$ in $83 \%$ yield according to the similar procedures.

Dehydro[10]paracyclophane: colorless oil; ${ }^{1} \mathrm{H}$ NMR $(400 \mathrm{MHz}$, $\left.\mathrm{CDCl}_{3}\right) \delta=1.08-1.14(\mathrm{~m}, 2 \mathrm{H}), 1.20-1.31(\mathrm{~m}, 4 \mathrm{H}), 1.50-1.71(\mathrm{~m}, 6 \mathrm{H})$, $2.55-2.58(\mathrm{~m}, 2 \mathrm{H}), 2.67(\mathrm{t}, J=6.4 \mathrm{~Hz}, 2 \mathrm{H}), 4.51-4.54(\mathrm{~m}, 1 \mathrm{H})$, 4.96-4.99 (m, 1H), 7.08-7.12 (m, 4H); $\left.{ }^{13} \mathrm{C} \mathrm{NMR} \mathrm{(100} \mathrm{MHz,} \mathrm{CDCl}_{3}\right) \delta$

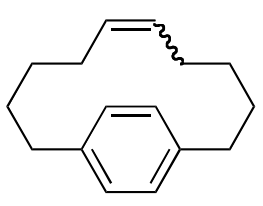
$=24.3,26.4,28.6,28.8,30.2,31.9,35.3,35.7,127.8,129.2,129.38,129.4,139.3$, 140.0; high-resolution MS (EI, $70 \mathrm{eV}$ ): calc. for $\mathrm{C}_{16} \mathrm{H}_{22}$ : 214.1721, found: 214.1717. 
Dehydro[11]paracyclophane: colorless oil; ${ }^{1} \mathrm{H}$ NMR $(500 \mathrm{MHz}$, $\left.\mathrm{CDCl}_{3}\right) \delta=0.69-0.77(\mathrm{~m}, 1 \mathrm{H}), 0.85-0.93(\mathrm{~m}, 1 \mathrm{H}), 1.01-1.08(\mathrm{~m}, 1 \mathrm{H})$, $1.12-1.43(\mathrm{~m}, 5 \mathrm{H}), 1.45-1.79(\mathrm{~m}, 6 \mathrm{H}), 2.50-2.54(\mathrm{~m}, 1 \mathrm{H}), 2.58-2.67$ $(\mathrm{m}, 3 \mathrm{H}), 4.65-4.83(\mathrm{~m}, 1 \mathrm{H}), 5.02-5.15(\mathrm{~m}, 1 \mathrm{H}), 7.05-7.13(\mathrm{~m}, 4 \mathrm{H})$;

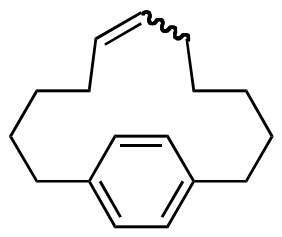
${ }^{13} \mathrm{C}$ NMR (125 MHz, $\left.\mathrm{CDCl}_{3}\right) \delta=23.6,25.5,27.7,29.7,30.1,30.2,31.3,35.1$ (2C), 128.8, 129.0, 129.27, 129.33, 130.1, 130.2, 140.1, 140.2; MS (EI); m/z (\%): 228 (100) $\left[M^{+}\right]$; high-resolution MS (EI, $70 \mathrm{eV}$ ): calc. for $\mathrm{C}_{17} \mathrm{H}_{24}: 228.1877$, found: 228.1875 .

Preparation of [10]- and [11]-paracyclophanes $(5 \mathrm{c}, \mathrm{d})$. Representative Procedure. A solution of dehydro[11]- paracyclophane $(313 \mathrm{mg}, 1.37 \mathrm{mmol})$ in ethyl acetate $(50 \mathrm{ml})$ was stirred in hydrogen atmosphere in the presence of $10 \mathrm{wt} \% \mathrm{Pd} / \mathrm{C}(60 \mathrm{mg})$ at room temperature for $30 \mathrm{~min}$. After the mixture was filtered through celite, the filtrate was concentrated in vacuo and separated by flash chromatography on silica gel with hexane to produce the known [11]paracyclophane (5d) (312 mg, 99\%). Hydrogenation of 3c under the similar reaction conditions afforded the known [10]paracyclophane (5c) in $96 \%$ yield.

[10]Paracyclophane (5c): ${ }^{1}$ colarless oil: ${ }^{1} \mathrm{H} \mathrm{NMR}\left(400 \mathrm{MHz}, \mathrm{CDCl}_{3}\right) \delta$ $=0.45-0.58(\mathrm{~m}, 4 \mathrm{H}), 0.67-0.80(\mathrm{~m}, 4 \mathrm{H}), 1.00-1.14(\mathrm{~m}, 4 \mathrm{H}), 1.55(\mathrm{q}, J=$ $6.30 \mathrm{~Hz}, 4 \mathrm{H}), 2.64(\mathrm{t}, J=6.30 \mathrm{~Hz}, 4 \mathrm{H}), 7.10(\mathrm{~s}, 4 \mathrm{H}) ;{ }^{13} \mathrm{C} \mathrm{NMR}(100$ $\left.\mathrm{MHz}, \mathrm{CDCl}_{3}\right) \delta=25.0,26.9,28.4,29.2,35.3,129.3,139.6$.

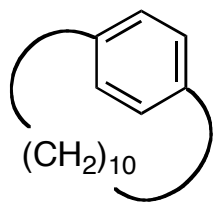

[11]Paracyclophane (5d): ${ }^{2}$ colorless oil: ${ }^{1} \mathrm{H} \mathrm{NMR}\left(500 \mathrm{MHz}, \mathrm{CDCl}_{3}\right)$ $\delta=0.63$ (quint, $J=6.5 \mathrm{~Hz}, 2 \mathrm{H}$ ), 0.76-0.87 (m, 8H), 1.19 (quint, $J=6.5$ $\mathrm{Hz}, 4 \mathrm{H}), 1.36-1.60(\mathrm{~m}, 4 \mathrm{H}), 2.57-2.60(\mathrm{~m}, 4 \mathrm{H}), 7.10(\mathrm{~s}, 4 \mathrm{H}) ;{ }^{13} \mathrm{C} \mathrm{NMR}$ $\left(125 \mathrm{MHz}, \mathrm{CDCl}_{3}\right) \delta=25.5$ (2C), 26.7 (2C), 27.4, 28.2 (2C), $29.0(2 \mathrm{C})$,

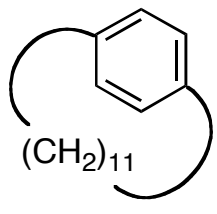
35.8 (2C), 129.0 (4C), 140.3 (2C).

Paracyclophanecarboxylic acid 6c,d. The compounds 6c,d was prepared from 5c,d based on the similar procedure reported in literature. ${ }^{3}$ Representative Procedure. Bromine $(0.251 \mathrm{ml}, 4.872 \mathrm{mmol})$ was dissolved in carbon tetrachloride $(22 \mathrm{ml})$ and the

(1) Otsubo, T.; Misumi, S. Synth. Commun. 1978, 8, 285-289.

(2) Hochmuth, D. H.; König, W. A. Tetrahedron: Asymmetry 1999, 10, 1089-1097.

(3) (a) Oi, S.; Miyano, S. Chem. Lett. 1992, 987-990. (b) Cram, D. J.; Day, A. C. J. Org. Chem. 1966, 31, 1227-1232. 
solution $(2 \mathrm{ml})$ was added to iron power $(13.6 \mathrm{mg}, 0.244 \mathrm{mmol})$ suspended in dichloromethane $(9.0 \mathrm{ml})$. After stirring at room temperature for $2 \mathrm{~h}$, the mixture was diluted in dichloromethane $(70 \mathrm{ml})$ and was heated at $60{ }^{\circ} \mathrm{C}$. To the mixture was added a solution of $\mathbf{5 c}(877.7 \mathrm{mg}, 4.06 \mathrm{mmol})$ in dichloromethane $(9.0 \mathrm{ml})$ and the remaining bromine solution $(20 \mathrm{ml})$ was added dropwise at $60{ }^{\circ} \mathrm{C}$ during $6 \mathrm{~h}$. After stirring for further $14 \mathrm{~h}$, the mixture was diluted in dichloromethane and was washed with $10 \%$ aqueous sodium thiosulfate, water, and brine. After removal of solvent in vacuo, the residue was purified by flash chromatography on silica gel with hexane to give 12-bromo[10]paracyclophane ${ }^{3 \mathrm{a}}(1.15 \mathrm{~g}, 96 \%)$ as a colorless oil: ${ }^{1} \mathrm{H}$ NMR (400 MHz, $\left.\mathrm{CDCl}_{3}\right) \delta=0.28-0.39(\mathrm{~m}, 1 \mathrm{H}), 0.43-0.53(\mathrm{~m}, 1 \mathrm{H})$, 0.64-0.90 (m, 7H), 0.94-1.32 (m, 4H), 1.40-1.49 (m, 1H), 1.57-1.73 (m, 2H), 2.47 (ddd, $J=5.16,9.74,13.17 \mathrm{~Hz}, 1 \mathrm{H}), 2.54$ (ddd, $J=4.58$,

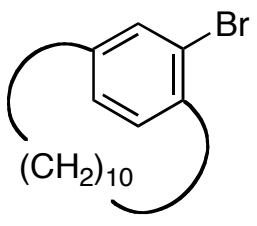
9.16, 13.17 Hz, 1H), 2.67 (ddd, $J=4.58,6.30,13.17 \mathrm{~Hz}, 1 \mathrm{H}), 3.11(\mathrm{td}, J=5.16,13.17$ $\mathrm{Hz}, 1 \mathrm{H}), 7.03(\mathrm{dd}, J=1.72,7.45 \mathrm{~Hz}, 1 \mathrm{H}), 7.11(\mathrm{~d}, J=7.45 \mathrm{~Hz}, 1 \mathrm{H}), 7.37$ (brs, $1 \mathrm{H}) ;{ }^{13} \mathrm{C}$ NMR $\left(100 \mathrm{MHz}, \mathrm{CDCl}_{3}\right) \delta=24.8,24.8,26.3,26.8,26.9,28.3,28.3,28.9,34.9,35.3$, 128.6, 131.5, 133.3, 135.1, 138.5, 142.1. high-resolution MS (EI, $70 \mathrm{eV}$ ): calc. for $\mathrm{C}_{16} \mathrm{H}_{23}{ }^{81} \mathrm{Br}$ : 296.0963, found: 296.0941. The bromocyclophane thus obtained (1.15 g, $3.90 \mathrm{mmol})$ was dissolved in THF $(40 \mathrm{ml})$ and was cooled to $-78 \mathrm{C}$. $1.47 \mathrm{M}$ tert-Butyllithium solution in hexane $(5.96 \mathrm{ml}, 8.58 \mathrm{mmol})$ was added dropwise during $15 \mathrm{~min}$ by a syringe and the mixture was stirred at the same temperature for further $1 \mathrm{~h}$. Carbon dioxide was then passed through the reaction mixture for $1 \mathrm{~h}$ and the whole mixture was raised to room temperature under carbon dioxide atmosphere. After $1 \mathrm{~N}$ hydrochloric acid $(20 \mathrm{ml})$ was added and stirred for $30 \mathrm{~min}$, ether was added and the aqueous layer was further extracted with ether. The combined ethereal layer was concentrated in vacuo and the residue was separated by flash chromatography on silica gel with hexane-ethyl acetate (10/1) to give acid $\mathbf{6 c}(808.9 \mathrm{mg}, 80 \%)$.

[10]Paracyclophane-12-carboxylic acid (6c): ${ }^{3 \mathrm{a}, 4}$ white crystal: mp 135.6-137.1 ${ }^{\circ} \mathrm{C}$ (lit. $\left.{ }^{3 \mathrm{a}} 140{ }^{\circ} \mathrm{C}\right) ;{ }^{1} \mathrm{H} \mathrm{NMR}\left(500 \mathrm{MHz}, \mathrm{CDCl}_{3}\right) \delta=$ $0.05-0.25(\mathrm{~m}, 1 \mathrm{H}), 0.38-0.56(\mathrm{~m}, 1 \mathrm{H}), 0.56-0.84(\mathrm{~m}, 5 \mathrm{H})$, 0.84-1.36 (m, 5H), 1.39-1.60 (m, 2H), 1.63-1.84 (m, 2H), 2.43

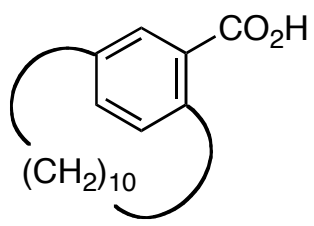

(4) Harada, N.; Soutome, T.; Nehira, T.; Uda, H.; Oi, S.; Okamura, A.; Miyano, S. J. Am. Chem. Soc. 1993, 115, 7547-7548. 
(ddd, $J=4.62,10.55,12.86 \mathrm{~Hz}, 1 \mathrm{H}), 2.62(\mathrm{ddd}, J=4.62,9.23,13.19 \mathrm{~Hz}, 1 \mathrm{H}), 2.77$ (ddd, $J=4.95,6.59,13.19 \mathrm{~Hz}, 1 \mathrm{H}), 3.83(\mathrm{td}, J=4.95,12.86 \mathrm{~Hz}, 1 \mathrm{H}), 7.23$ (d, $J=7.58$ $\mathrm{Hz}, 1 \mathrm{H}), 7.28(\mathrm{dd}, J=1.98,7.58 \mathrm{~Hz}, 1 \mathrm{H}), 7.93(\mathrm{~d}, J=1.98 \mathrm{~Hz}, 1 \mathrm{H}) ;{ }^{13} \mathrm{C} \mathrm{NMR}(125$ $\left.\mathrm{MHz}, \mathrm{CDCl}_{3}\right) \delta=24.7,25.3,26.7,26.8,28.1$, 28.4, 28.5, 28.7, 34.4, 34.9, 128.4, 132.5, 133.9, 140.2, 142.9, 172.8. high-resolution MS (EI, $70 \mathrm{eV}$ ): calc. for $\mathrm{C}_{17} \mathrm{H}_{24} \mathrm{O}_{2}$ : 260.1776, found: 260.1779 .

[11]Paracyclophane-13-carboxylic acid (6d): 69\% yield (2 steps from 5d); white solid; mp 136.6-137.8 ${ }^{\circ} \mathrm{C}$; ${ }^{1} \mathrm{H}$ NMR $(400 \mathrm{MHz}$, $\left.\mathrm{CDCl}_{3}\right) \delta=0.65-0.70(\mathrm{~m}, 3 \mathrm{H}), 0.78-0.88(\mathrm{~m}, 7 \mathrm{H}), 1.11-1.62(\mathrm{~m}$, $1 \mathrm{H}), 1.21-1.28(\mathrm{~m}, 3 \mathrm{H}), 1.54-1.76(\mathrm{~m}, 4 \mathrm{H}), 2.43(\mathrm{ddd}, J=13.0,6.5$,

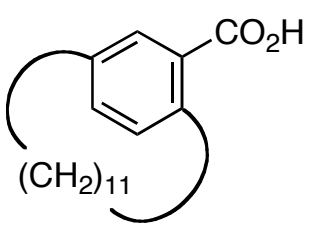
$3 \mathrm{~Hz}, 1 \mathrm{H}), 2.61(\mathrm{ddd}, J=13.0,7.5,5.5 \mathrm{~Hz}, 1 \mathrm{H}), 2.70(\mathrm{ddd}, J=13.0,7.5,5.5 \mathrm{~Hz}, 1 \mathrm{H})$ $3.78(\mathrm{ddd}, J=13.0,6.5,3 \mathrm{~Hz}, 1 \mathrm{H}), 7.20$ (d, $J=8 \mathrm{~Hz}, 1 \mathrm{H}), 7.30(\mathrm{dd}, J=1.5 \mathrm{~Hz}, 1 \mathrm{H})$, $7.90(\mathrm{~d}, J=1.5 \mathrm{~Hz}, 1 \mathrm{H}) ;{ }^{13} \mathrm{C} \mathrm{NMR}\left(100 \mathrm{MHz}, \mathrm{CDCl}_{3}\right) \delta=25.6,25.7,26.5,26.7,27.0$, 27.3, 28.0, 28.2, 28.7, 34.1, 35.5, 126.3, 132.1, 132.4, 133.5, 140.9, 143.3, 172.9; high-resolution $\mathrm{MS}$ (EI, $70 \mathrm{eV}$ ): calc. for $\mathrm{C}_{18} \mathrm{H}_{26} \mathrm{O}_{2}$ : 274.1933, found: 274.1937. 13 -Bromo[11] paracyclophane, a precursor of $\mathbf{6 d}$, was reported previously in literature. ${ }^{2}$

Synthesis and diastereomeric resolution of 7c,d and 8d. Representative Procedure. The compound $\mathbf{6 c}(300 \mathrm{mg}, 1.15 \mathrm{mmol})$ and oxalyl chloride $(0.301 \mathrm{ml}, 3.45 \mathrm{mmol})$ were stirred in chloroform $(10.0 \mathrm{ml})$ at room temperature for $1.5 \mathrm{~h}$. After removal of solvent in vacuo, the residue was dissolved in chloroform $(10.0 \mathrm{ml})$ and the mixture was added dropwise at $0{ }^{\circ} \mathrm{C}$ to a solution of $(S)$-valinol $(179 \mathrm{mg}, 1.73 \mathrm{mmol})$ and triethyl amine $(0.241 \mathrm{ml}, 1.73 \mathrm{mmol})$ in chloroform $(10.0 \mathrm{ml})$. After stirring at room temperature for $12 \mathrm{~h}$, water was added and the aqueous layer was extracted with chloroform. The combined organic layer was washed with brine and was concentrated in vacuo to give a crude diastereomeric mixture of $\left(\boldsymbol{R}_{\mathrm{p}}, \boldsymbol{S}\right)$-7c and $\left(\boldsymbol{S}_{\mathrm{p}}, \boldsymbol{S}\right)$-7c. The mixture was separated by MPLC on silica gel with hexane-ether (1/1) as an eluent to give [10]paracyclophane derivatives $\left(\boldsymbol{R}_{\mathrm{p}}, \boldsymbol{S}\right)-\mathbf{7 c}(179.8 \mathrm{mg}, 45 \%,>99 \% \mathrm{de})$ and $\left(\boldsymbol{S}_{\mathrm{p}}\right.$, $S)-7 c$ (177.3 mg, 45\%, 99\% de). Their diastereomeric excesses were determined by using HPLC (PEGASIL Silica 120-5) with 20\% ethyl acetate in hexane as an eluent. [11]Paracyclophane derivatives $\left(\boldsymbol{R}_{\mathrm{p}}, \boldsymbol{S}\right)-\mathbf{7 d}, \mathbf{8 d}$ and $\left(\boldsymbol{S}_{\mathrm{p}}, \boldsymbol{S}\right)-\mathbf{7 d}, \mathbf{8 d}$ were prepared according to the similar procedure. 
(S)-N-(1-Hydroxy-3-methyl-2-butanyl)-(R)-[10]paracyclophane-12-carboxamide $\left[\left(R_{\mathrm{p}}, S\right)-7 \mathrm{c}\right]$ : white solid; m.p. $110.8-113.9{ }^{\circ} \mathrm{C} ;{ }^{1} \mathrm{H}$ NMR $\left(400 \mathrm{MHz}, \mathrm{CDCl}_{3}\right) \delta=$ 0.31-0.39 (m, 1H), 0.41-0.50 (m, 1H), 0.55-0.72 (m, 2H),

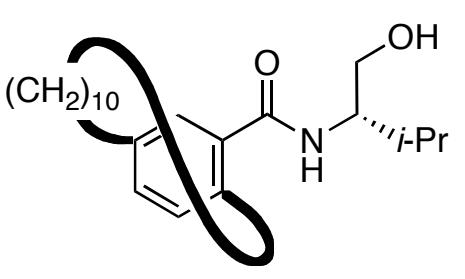
$0.72-0.81(\mathrm{~m}, 4 \mathrm{H}), 1.01(\mathrm{~d}, J=6.8 \mathrm{~Hz}, 3 \mathrm{H}), 1.04(\mathrm{~d}, J=$ $6.8 \mathrm{~Hz}, 3 \mathrm{H}), 0.96-1.20(\mathrm{~m}, 4 \mathrm{H}), 1.45-1.72(\mathrm{~m}, 4 \mathrm{H}), 2.00(8, J=6.8 \mathrm{~Hz}, 1 \mathrm{H}), 2.49$ (ddd, $J=13.2,8.8,4.8 \mathrm{~Hz}, 1 \mathrm{H}), 2.56$ (brs, $1 \mathrm{H}), 2.60$ (ddd, $J=13.2,8.8,4.8 \mathrm{~Hz}, 1 \mathrm{H}), 2.70$ (ddd, $J=13.2,6.8,4.8 \mathrm{~Hz}, 1 \mathrm{H}), 3.22$ (ddd, $J=13.2,6.8,5.2 \mathrm{~Hz}, 1 \mathrm{H}), 3.73-3.80(\mathrm{~m}, 2 \mathrm{H})$, 3.90-3.95 (m, 1H), $5.93(\mathrm{~d}, J=7.5 \mathrm{~Hz}, 1 \mathrm{H}), 7.14-7.18(\mathrm{~m}, 2 \mathrm{H}), 7.24-7.25(\mathrm{~m}, 1 \mathrm{H}) ;{ }^{13} \mathrm{C}$ NMR $\left(100 \mathrm{MHz}, \mathrm{CDCl}_{3}\right) \delta=18.8,19.7,25.03,25.05,26.8,26.9,28.2,28.3,28.5,28.9$, $29.2,32.9,35.0,57.7,64.3,128.0,131.1,131.4,136.8,137.6,140.2,171.4$; CD $\left(\mathrm{CH}_{3} \mathrm{CN}\right): \lambda_{\mathrm{ext}}=233.2(\Delta \varepsilon+14.9), 206.0(\Delta \varepsilon-20.2) ;[\alpha]_{\mathrm{D}}{ }^{23}=-11.6\left(\mathrm{c}=1.1\right.$ in $\left.\mathrm{CHCl}_{3}\right)$; high-resolution MS (EI, $70 \mathrm{eV}$ ): calc. for $\mathrm{C}_{22} \mathrm{H}_{35} \mathrm{NO}_{2}$ : 345.2668, found: 345.2672 .

(S)-N-(1-Hydroxy-3-methyl-2-butanyl)-(S)-[10]paracyclophane-12-carboxamide $\left[\left(S_{\mathrm{p}}, S\right)-7 \mathrm{c}\right]$ : colorless oil; ${ }^{1} \mathrm{H}$ NMR $\left(400 \mathrm{MHz}, \mathrm{CDCl}_{3}\right) \delta=0.33-0.41(\mathrm{~m}, 1 \mathrm{H})$, 0.43-0.51 (m, 1H) 0.58-0.70 (m, 2H), 0.72-0.81 (m, 4H), $1.01(\mathrm{~d}, J=6.8 \mathrm{~Hz}, 3 \mathrm{H}), 1.03(\mathrm{~d}, J=6.8 \mathrm{~Hz}, 3 \mathrm{H})$,

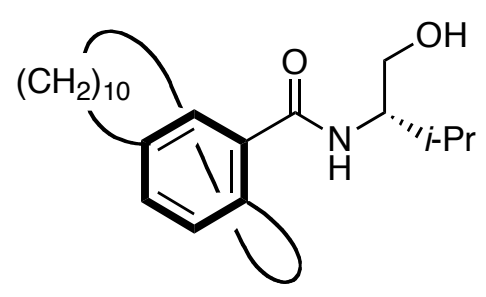
1.07-1.22 (m, 4H), 1.48-1.69 (m, 4H), $2.00(8, J=6.8 \mathrm{~Hz}, 1 \mathrm{H}), 2.50$ (ddd, $J=13.2,8.8$, 4.8, 1H), 2.61 (ddd, $J=13.2,8.8,4.8,1 \mathrm{H}), 2.71(\mathrm{ddd}, J=13.2,6.8,4.8 \mathrm{~Hz}, 1 \mathrm{H}), 3.23$ (ddd, $J=13.2,6.8,5.2 \mathrm{~Hz}, 1 \mathrm{H}), 3.79(\mathrm{dd}, J=11.0,5.5 \mathrm{~Hz}, 1 \mathrm{H}), 3.84(\mathrm{dd}, J=11.0,3.5$ $\mathrm{Hz}, 1 \mathrm{H}), 3.92-3.98(\mathrm{~m}, 1 \mathrm{H}), 5.89(\mathrm{~d}, J=7.5 \mathrm{~Hz}, 1 \mathrm{H}), 7.16(\mathrm{~s}, 2 \mathrm{H}), 7.25(\mathrm{~s}, 1 \mathrm{H}) ;{ }^{13} \mathrm{C}$ NMR $\left(100 \mathrm{MHz}, \mathrm{CDCl}_{3}\right) \delta=18.7,19.6,25.0,26.8,26.9,28.2,28.3,28.5,28.9,29.1$, $29.7,32.9,35.0,57.5,64.2,127.8,131.1,131.5,136.8,137.7,140.2,171.2$; CD $\left(\mathrm{CH}_{3} \mathrm{CN}\right): \lambda_{\text {ext }}=280.0(\Delta \varepsilon+0.57), 232.2(\Delta \varepsilon-9.6), 204.2(\Delta \varepsilon+12.1) ;[\alpha]_{\mathrm{D}}^{23}=-38.4(\mathrm{c}=$ 1.0 in $\mathrm{CHCl}_{3}$ ); $\mathrm{MS}(\mathrm{EI}): \mathrm{m} / z(\%): 345(6)\left[\mathrm{M}^{+}\right], 243$ (47), 69 (100); high-resolution MS (ESI+): calc. for $\mathrm{C}_{22} \mathrm{H}_{35} \mathrm{NNaO}_{2}$ : 368.2566, found: 368.2574 .

(S)-N-(1-Hydroxy-3-methyl-2-butanyl)-(R)-[11]paracyclophane-13-carboxamide $\left[\left(\boldsymbol{R}_{\mathrm{p}}, \boldsymbol{S}\right)-\mathbf{7 d}\right]: 47 \%$ yield (99\% de); white solid; m.p. 108.0-110.9 ${ }^{\circ} \mathrm{C} ;{ }^{1} \mathrm{H}$ NMR $\left(400 \mathrm{MHz}, \mathrm{CDCl}_{3}\right) \delta=0.65-0.92(\mathrm{~m}, 10 \mathrm{H}), 1.02(\mathrm{~d}, J=$ $6.9 \mathrm{~Hz}, 3 \mathrm{H}), 1.05(\mathrm{t}, J=6.9 \mathrm{~Hz}, 3 \mathrm{H}), 1.11-1.29(\mathrm{~m}, 4 \mathrm{H})$,

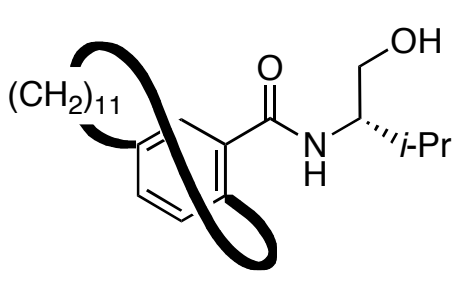
1.57-1.64 (m, 4H), 2.01 (octet, $J=6.9 \mathrm{~Hz}, 1 \mathrm{H}), 2.47$ (ddd, $J=13.3,6.0,5.5 \mathrm{~Hz}, 1 \mathrm{H})$, 
2.60-2.63 (m, 2H), 2.66 (brs, 1H), 3.17-3.24 (m, 1H), 3.70-3.83 (m, 2H), 3.90-3.99 (m, $1 \mathrm{H}), 5.89(\mathrm{~d}, J=7.8 \mathrm{~Hz}, 1 \mathrm{H}), 7.18-7.21(\mathrm{~m}, 3 \mathrm{H}) ;{ }^{13} \mathrm{C} \mathrm{NMR}\left(100 \mathrm{MHz}, \mathrm{CDCl}_{3}\right) \delta=18.7$, 19.7, 25.6, 25.8, 26.8, 26.8, 27.3, 28.1, 28.2, 28.5, 28.9, 29.2, 32.9, 35.5, 57.7, 64.7, $127.7,130.7,131.2,136.6,138.3,140.9,171.6 ; \mathrm{CD}\left(\mathrm{CH}_{3} \mathrm{CN}\right): \lambda_{\mathrm{ext}}=231.4(\Delta \varepsilon+10.7)$, $202.2(\Delta \varepsilon-14.0) ;[\alpha]_{\mathrm{D}}{ }^{24}=-4.49\left(\mathrm{c}=1.0\right.$ in $\left.\mathrm{CHCl}_{3}\right)$; high-resolution MS (EI, $\left.70 \mathrm{eV}\right)$ : calc. for $\mathrm{C}_{23} \mathrm{H}_{37} \mathrm{NO}_{2}$ : 359.2824, found: 359.2825 .

(S)-N-(1-Hydroxy-3-methyl-2-butanyl)-(S)-[11]paracyclophane-13-carboxamide $\left[\left(S_{\mathrm{p}}, \boldsymbol{S}\right)-7 \mathrm{~d}\right]: 37 \%$ yield (>99\% de); white solid; m.p. 82.2-83.8 ${ }^{\circ} \mathrm{C} ;{ }^{1} \mathrm{H}$ NMR $\left(400 \mathrm{MHz}, \mathrm{CDCl}_{3}\right) \delta=0.61-0.94(\mathrm{~m}, 10 \mathrm{H}), 1.01(\mathrm{~d}, J=$ $7.8 \mathrm{~Hz}, 3 \mathrm{H}), 1.03$ (d, $J=7.8 \mathrm{~Hz}, 3 \mathrm{H}), 1.13-1.26(\mathrm{~m}, 4 \mathrm{H})$,

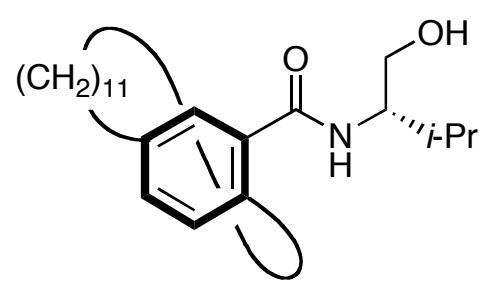
1.60 (quint, $J=6.9 \mathrm{~Hz}, 4 \mathrm{H}$ ), 1.99 (octet, $J=6.9 \mathrm{~Hz}, 1 \mathrm{H}$ ), 2.48 (ddd, $J=13.3,7.3,6.4$ $\mathrm{Hz}, 1 \mathrm{H}), 2.55-2.66$ (m, 2H), 2.74 (brs, 1H), 3.19 (ddd, $J=13.3,6.9,4.6 \mathrm{~Hz}, 1 \mathrm{H}), 3.77$ $(\mathrm{dd}, J=11.0,6.0 \mathrm{~Hz}, 1 \mathrm{H}), 3.83(\mathrm{dd}, J=11.0,3.7 \mathrm{~Hz}, 1 \mathrm{H}), 3.84-3.99(\mathrm{~m}, 1 \mathrm{H}), 5.90(\mathrm{~d}, J$ $=8.2,1 \mathrm{H}), 7.18-7.26(\mathrm{~m}, 3 \mathrm{H}) ;{ }^{13} \mathrm{C} \mathrm{NMR}\left(100 \mathrm{MHz}, \mathrm{CDCl}_{3}\right) \delta=18.7,19.6,25.6,25.7$, 26.7, 26.8, 27.3, 28.1, 28.2, 28.6, 28.8, 29.1, 32.8, 35.6, 57.5, 64.4, 127.6, 130.6, 131.2, 136.6, 138.3, 140.9, 171.5; $\mathrm{CD}\left(\mathrm{CH}_{3} \mathrm{CN}\right): \lambda_{\mathrm{ext}}=279.9(\Delta \varepsilon+0.28), 231.0(\Delta \varepsilon-11.4)$, $206.1(\Delta \varepsilon+15.7) ;[\alpha]_{\mathrm{D}}{ }^{24}=-29.8\left(\mathrm{c}=0.5\right.$ in $\left.\mathrm{CHCl}_{3}\right)$; MS (EI): $m / z(\%): 359(70)\left[\mathrm{M}^{+}\right]$, 149 (100); high-resolution MS (EI, $70 \mathrm{eV}$ ): calc. for $\mathrm{C}_{23} \mathrm{H}_{37} \mathrm{NO}_{2}$ : 359.2824, found: 359.2830 .

(S)-N-(1-Hydroxy-3,3-dimethyl-2-butanyl)-(R)-[11]paracyclophane-13-carboxamide $\left[\left(R_{\mathrm{p}}, S\right)-8 \mathrm{~d}\right]: 46 \%$ yield (>99\% de); white solid; m.p. $155.9-161.2{ }^{\circ} \mathrm{C} ;{ }^{1} \mathrm{H}$ NMR $\left(400 \mathrm{MHz}, \mathrm{CDCl}_{3}\right) \delta=0.56-0.92(\mathrm{~m}, 10 \mathrm{H}), 0.98(\mathrm{~s}$, 9H), 1.04-1.49 (m, 4H), 1.51-1.59 (m, 4H), 2.42 (ddd, $J=$

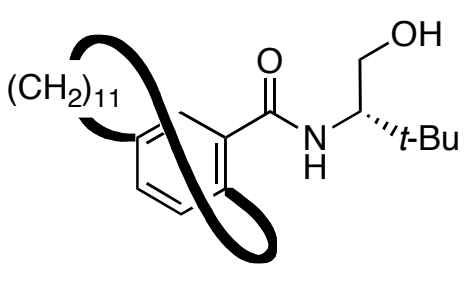
12.6, 7.5, 4.6 Hz, 1H), 2.67 (t, $J=5.2 \mathrm{~Hz}, 2 \mathrm{H}), 3.15$ (ddd, $J=11.5,6.9,4.6 \mathrm{~Hz}, 1 \mathrm{H}$ ), 3.59 (ddd, $J=11.5,7.5,4.0 \mathrm{~Hz}, 1 \mathrm{H}), 3.86-3.96(\mathrm{~m}, 2 \mathrm{H}), 5.80(\mathrm{~d}, J=8.0 \mathrm{~Hz}, 1 \mathrm{H})$, 7.10-7.14 (m, 3H); ${ }^{13} \mathrm{C}$ NMR (100 MHz, $\left.\mathrm{CDCl}_{3}\right) \delta=25.6,25.8,26.8,26.8,27.1$ (3C), 27.4, 28.2, 28.2, 28.4, 28.9, 32.8, 33.6, 35.6, 60.5, 64.1, 127.6, 130.7, 131.3, 138.3, 139.0, 140.9, 172.0: $\lambda_{\mathrm{ext}}=231.7(\Delta \varepsilon+9.7), 205.1(\Delta \varepsilon-12.4) ;[\alpha]_{\mathrm{D}}{ }^{23}=-1.4(\mathrm{c}=1.0$ in $\mathrm{CHCl}_{3}$ ); high-resolution $\mathrm{MS}(\mathrm{EI}, 70 \mathrm{eV})$ : calc. for $\mathrm{C}_{24} \mathrm{H}_{39} \mathrm{NO}_{2}$ : 373.2981, found: 373.2974 . 
(S)-N-(1-Hydroxy-3,3-dimethyl-2-butanyl)-(S)-[11]paracyclophane-13-carboxamide $\left[\left(S_{\mathrm{p}}, S\right)-8 \mathrm{~d}\right]: 48 \%$ yield (98\% de); colorless oil; ${ }^{1} \mathrm{H}$ NMR (400 MHz, $\left.\mathrm{CDCl}_{3}\right) \delta=0.56-0.92(\mathrm{~m}, 10 \mathrm{H}), 0.98(\mathrm{~s}, 9 \mathrm{H}), 1.04-1.40$

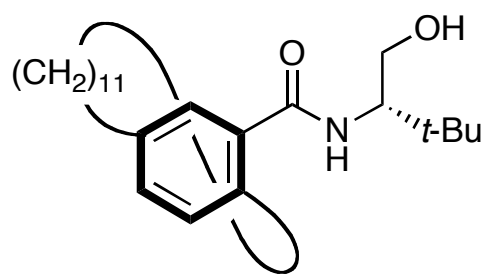
(m, 4H), 1.51-1.61 (m,4H), $2.41(\mathrm{ddd}, J=13.8,8.6,4.6 \mathrm{~Hz}, 1 \mathrm{H}), 2.49-2.58(\mathrm{~m}, 2 \mathrm{H})$, $3.13(\mathrm{ddd}, J=13.8,7.5,4.6 \mathrm{~Hz}, 1 \mathrm{H}), 3.60(\mathrm{dd}, J=10.9,8.0 \mathrm{~Hz}, 1 \mathrm{H}), 3.90-3.98$ (m, 2H), $5.78(\mathrm{~d}, J=8.0 \mathrm{~Hz}, 1 \mathrm{H}), 7.12-7.14(\mathrm{~m}, 3 \mathrm{H}) ;{ }^{13} \mathrm{C} \mathrm{NMR}\left(100 \mathrm{MHz}, \mathrm{CDCl}_{3}\right) \delta=25.5,25.6$, 26.7, 27.0 (3C), 27.4, 28.1, 28.2, 28.8, 29.7, 31.6, 32.8, 33.5, 35.5, 60.1, 68.1, 127.5, $130.6,131.2,136.7,138.3,140.9,171.8: \lambda_{\text {ext }}=279.0(\Delta \varepsilon+0.23), 230.9(\Delta \varepsilon-7.2), 203.5$ $(\Delta \varepsilon+10.2) ;[\alpha]_{\mathrm{D}}^{23}=-18.1\left(\mathrm{c}=1.0\right.$ in $\left.\mathrm{CHCl}_{3}\right)$; high-resolution MS (EI, $\left.70 \mathrm{eV}\right)$ : calc. for $\mathrm{C}_{24} \mathrm{H}_{39} \mathrm{NO}_{2}$ : 373.2981, found: 373.2978 .

Crystallization-induced asymmetric transformation of $7 \mathbf{d}$ and $8 \mathrm{~d}$. Representative Procedure. A diastereomeric mixture of $\mathbf{8 d}(101.1 \mathrm{mg}, 0.271 \mathrm{mmol})$ was heated without solvent in a recovery flask $\left(5 \mathrm{ml}\right.$ vol.) at $110{ }^{\circ} \mathrm{C}$ for $2 \mathrm{~h}$ to afford $\left(\boldsymbol{R}_{\mathrm{p}}, \boldsymbol{S}\right)-\mathbf{8 d}$ with $95 \%$ $d e$. The $\left(\boldsymbol{R}_{\mathbf{p}}, \boldsymbol{S}\right)-\mathbf{8 d}$ rich mixture was further heated at $110^{\circ} \mathrm{C}$ for additional $2 \mathrm{~h}$ to give a nearly single diastereomer, $\left(\boldsymbol{R}_{\mathrm{p}}, \boldsymbol{S}\right)-\mathbf{8 d}(100.5 \mathrm{mg}, 99 \%)$ with $>99 \%$ de. The diastereomeric excess was determined by using HPLC (PEGASIL Silica 120-5) with $20 \%$ ethyl acetate in hexane as an eluent.

Synthesis of planar-chiral cyclophane esters $(\boldsymbol{R})-(+)-$ and $(S)-(-)-9 c$. Representative Procedure. To a solution of $\left(S_{\mathbf{p}}, S\right)-7 \mathrm{c}(320.0 \mathrm{mg}, 0.926 \mathrm{mmol})$ in dimethyl carbonate $(10.0 \mathrm{ml})$ was added sodium methoxide $(506 \mathrm{mg}, 9.37 \mathrm{mmol})$ and the mixture was stirred at room temperature for $30 \mathrm{~min}$. After the reaction was complete, water was added, the aqueous layer was extracted with ether, and the combined ethereal layer was concentrated in vacuo. The residue was separated by flash chromatography on silica gel with hexane-ether (30/1) to afford $(\boldsymbol{S})-(+)-9 c(244 \mathrm{mg}, 96 \%)$. Yield and reaction conditions for $(S)-(-)-9 c$ were shown in Scheme 4.

$(R)-(+)-[10]$ Paracyclophane-12-carboxylic acid methyl ester $[(\boldsymbol{R})-(+)-9 \mathrm{c}]:{ }^{5}$ colorless oil; ${ }^{1} \mathrm{H}$ NMR $(500 \mathrm{MHz}$, $\left.\mathrm{CDCl}_{3}\right) \delta=0.15(\mathrm{~m}, 1 \mathrm{H}), 0.46(\mathrm{~m}, 1 \mathrm{H}), 0.61(\mathrm{~m}, 1 \mathrm{H})$,

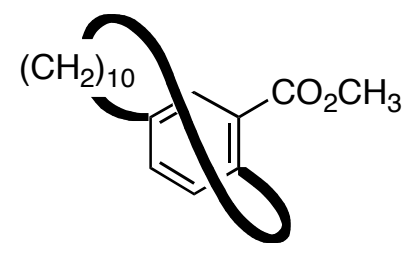

(5) Eberhardt, H.; Schlögl, K. Liebigs Ann. Chem. 1972, 760, 157-170. 
0.65-0.79 (m, 5H), $0.94(\mathrm{~m}, 1 \mathrm{H}), 1.06(\mathrm{~m}, 1 \mathrm{H}), 1.11-1.23(\mathrm{~m}, 2 \mathrm{H}), 1.34-1.55(\mathrm{~m}, 2 \mathrm{H})$, 1.68-1.73 (m, 2H), 2.40 (ddd, $J=12.7,10.7,4.9 \mathrm{~Hz}, 1 \mathrm{H}), 2.62$ (ddd, $J=13.2,9.3,4.9$ $\mathrm{Hz}, 1 \mathrm{H}), 2.73(\mathrm{ddd}, J=13.2,6.8,4.9 \mathrm{~Hz}, 1 \mathrm{H}), 3.69(\mathrm{td}, J=12.7,4.9 \mathrm{~Hz}, 1 \mathrm{H}), 3.89$ (s, $3 \mathrm{H}), 7.15(\mathrm{~d}, J=7.8 \mathrm{~Hz}, 1 \mathrm{H}), 7.23(\mathrm{dd}, J=7.8,2.0 \mathrm{~Hz}, 1 \mathrm{H}), 7.75(\mathrm{~d}, J=2.0 \mathrm{~Hz}, 1 \mathrm{H})$; ${ }^{13} \mathrm{C}$ NMR $\left(100 \mathrm{MHz}, \mathrm{CDCl}_{3}\right) \delta=24.8,25.3,26.7,26.8,28.1,28.3,28.4,28.7,34.2$, 35.0, 51.1, 130.3, 131.7, 132.1, 132.8, 140.0, 141.7, 168.4; $\mathrm{CD}\left(\mathrm{CH}_{3} \mathrm{CN}\right): \lambda_{\text {ext }}=298(\Delta \varepsilon$ $+0.91), 238.3(\Delta \varepsilon+16.51), 210.9(\Delta \varepsilon-24.18) ;[\alpha]_{\mathrm{D}}{ }^{20}=+53.1\left(\mathrm{c}=1.0\right.$ in $\left.\mathrm{CHCl}_{3}\right)$.

(S)-(-)-[10]Paracyclophane-12-carboxylic acid methyl ester $[(\boldsymbol{S})-(-)-9 \mathrm{c}]:{ }^{4}$ colorless oil; $\mathrm{CD}\left(\mathrm{CH}_{3} \mathrm{CN}\right): \lambda_{\text {ext }}=298(\Delta \varepsilon$ -0.37), $238.2(\Delta \varepsilon-15.28), 212(\Delta \varepsilon+22.67) ;[\alpha]_{\mathrm{D}}^{21}=-54.91(\mathrm{c}$ $=1.0$ in $\left.\mathrm{CHCl}_{3}\right)$; high-resolution MS (EI, $\left.70 \mathrm{eV}\right)$ : calc. for $\mathrm{C}_{18} \mathrm{H}_{26} \mathrm{O}_{2}$ : 274.1933, found: 274.1933.

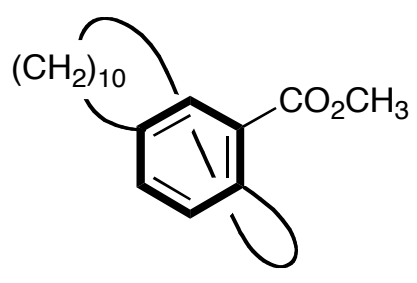

Synthesis of planar-chiral cyclophane ester $(R)-(+)-9 d$. To a solution of $\left(R_{p}, S\right)-8 d$ $(15.0 \mathrm{mg}, 0.040 \mathrm{mmol})$ in dichloromethane $(0.5 \mathrm{ml})$ and dimethyl carbonate $(2.5 \mathrm{ml})$ was added sodium methoxide $(32.4 \mathrm{mg}, 0.600 \mathrm{mmol})$ and 15 -crown-5 ether $(7.9 \mu \mathrm{l}$, $0.040 \mathrm{mmol}$ ) and the mixture was stirred at room temperature for $3 \mathrm{~h}$. Water was added, the aqueous layer was extracted with dichloromethane, and the combined organic layer was concentrated in vacuo. The residue was separated by flash chromatography on silica gel with hexane to afford $(\boldsymbol{R})-(+)-9 d(11.7 \mathrm{mg}$, quant) with $98.4 \%$ ee. The enantiomeric excess was determined by using HPLC (CHIRALPAK AS-RH, Dicel Chemical Ind., Ltd.) with acetonitrile-water (11/9) [(S)-(-)-9d: $25.02 \mathrm{~min},(\boldsymbol{R})-(+)-9 d$ : $28.68 \mathrm{~min}]$.

(R)-(+)-[11]paracyclophane-13-carboxylic acid methyl ester [(R)-(+)-9d]: colorless oil; ${ }^{1} \mathrm{H}$ NMR $\left(400 \mathrm{MHz}, \mathrm{CDCl}_{3}\right)$ $\delta=0.56-1.00(\mathrm{~m}, 8 \mathrm{H}), 1.00-1.46(\mathrm{~m}, 5 \mathrm{H}), 1.46-1.70(\mathrm{~m}, 5 \mathrm{H})$, 2.39 (ddd, $J=13.3,10.1,3.7 \mathrm{~Hz}, 1 \mathrm{H}), 2.55-2.69$ (m, 2H),

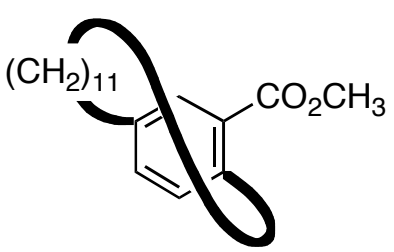
$3.63(\mathrm{ddd}, J=13.3,7.3,3.2 \mathrm{~Hz}, 1 \mathrm{H}), 3.89(\mathrm{~s}, 3 \mathrm{H}), 7.16(\mathrm{~d}, J=$ $7.8 \mathrm{~Hz}, 1 \mathrm{H}), 7.24(\mathrm{dd}, J=7.8,1.4 \mathrm{~Hz}, 1 \mathrm{H}), 7.75(\mathrm{~d}, J=1.4 \mathrm{~Hz}, 1 \mathrm{H}) ;{ }^{13} \mathrm{C} \mathrm{NMR}(100$ $\left.\mathrm{MHz}, \mathrm{CDCl}_{3}\right) \delta=25.5,25.7,26.6,26.7,27.3,28.0,28.2,28.8,28.8,33.9,35.5,51.8$, 129.6, 131.3, 131.8, 132.4, 140.7, 142.0, 168.5; $\mathrm{CD}\left(\mathrm{CH}_{3} \mathrm{CN}\right): \lambda_{\mathrm{ext}}=280.0(\Delta \varepsilon+0.99)$, $236.8(\Delta \varepsilon+18.96), 210.1(\Delta \varepsilon-26.38) ;[\alpha]_{\mathrm{D}}{ }^{15}=+55.2\left(\mathrm{c}=1.0\right.$ in $\left.\mathrm{CHCl}_{3}\right)$; high-resolution MS (EI, $70 \mathrm{eV}$ ): calc. for $\mathrm{C}_{19} \mathrm{H}_{28} \mathrm{O}_{2}$ : 288.2089, found: 288.2064. 
$F: \backslash 8 \% \ddot{E}<' \bullet \bullet \backslash 3 \frac{1}{4} \mathrm{E} f \mathrm{~A} f<f f f q f h \_P R O T O N .1$

K025-395-A-ECA500-CDCL3

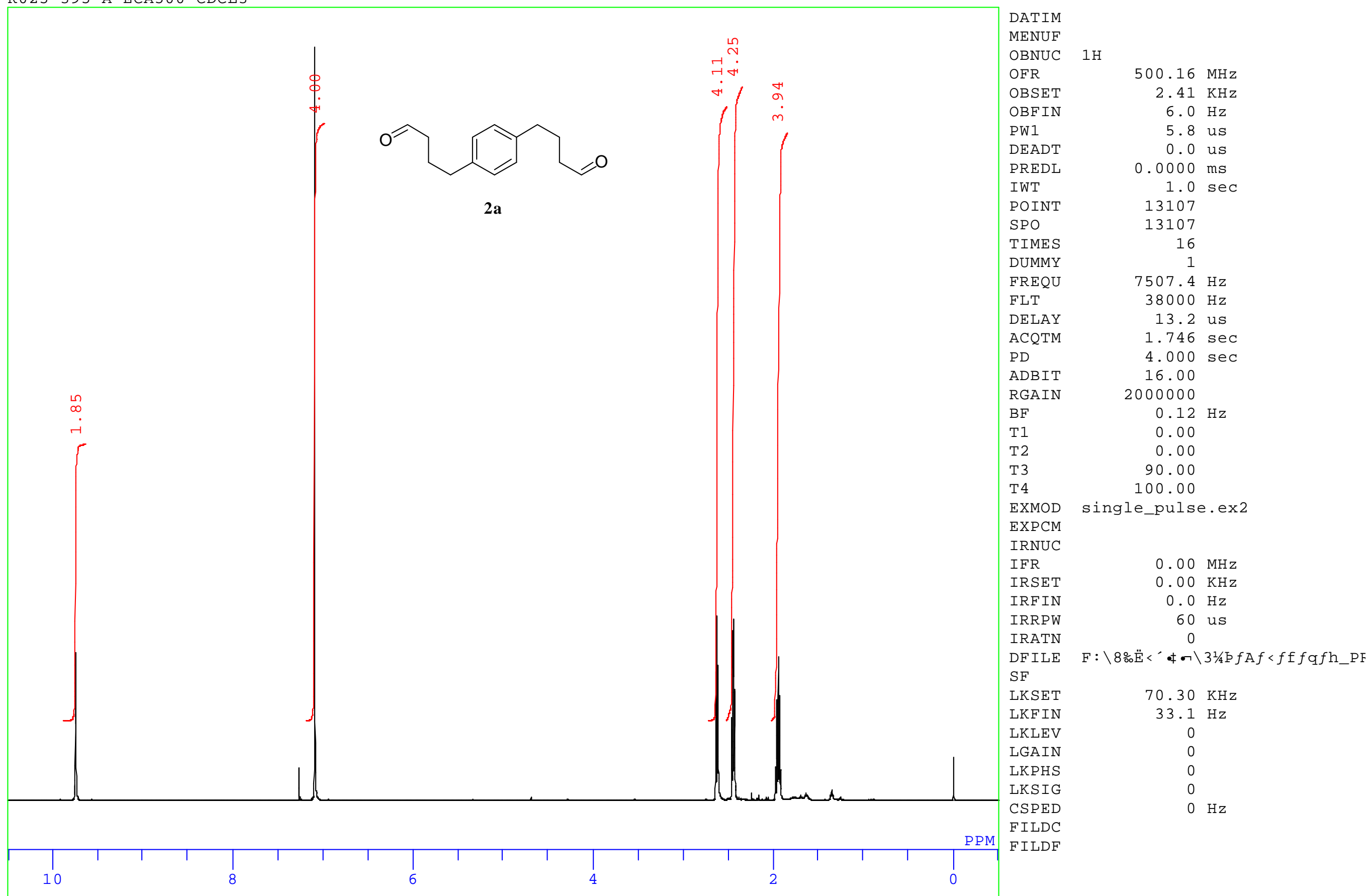


$F: \backslash 8 \% \ddot{E}<' \bullet \neq \neg \backslash 31 \frac{1}{4} \mathrm{E} f \mathrm{~A} f<f f f q f h \_C A R B O N .1$

K025-395-A-ECA 500-CDCL 3

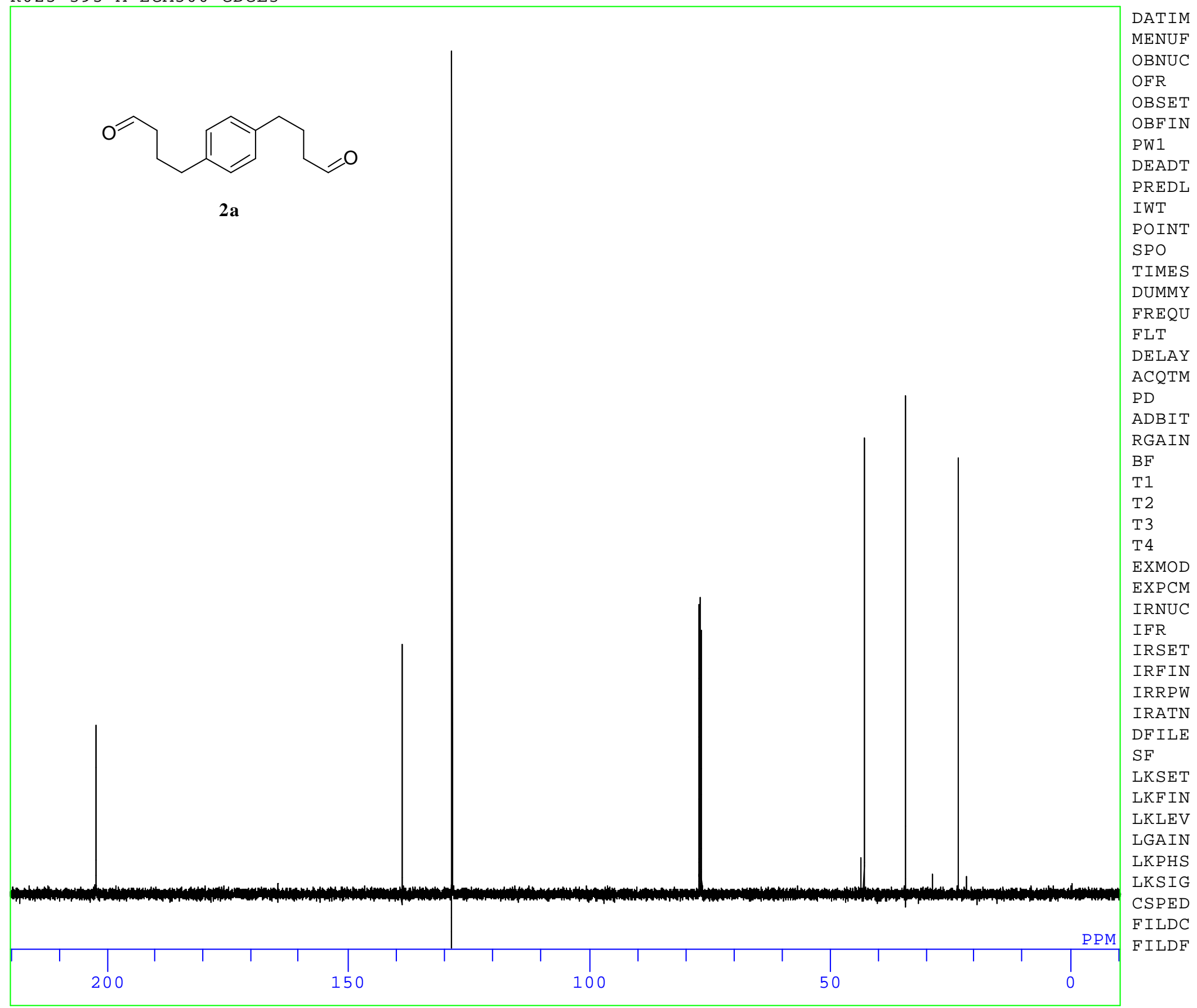


F : \9\%苂く'

K025-396-A-ECA 500-CDCL3

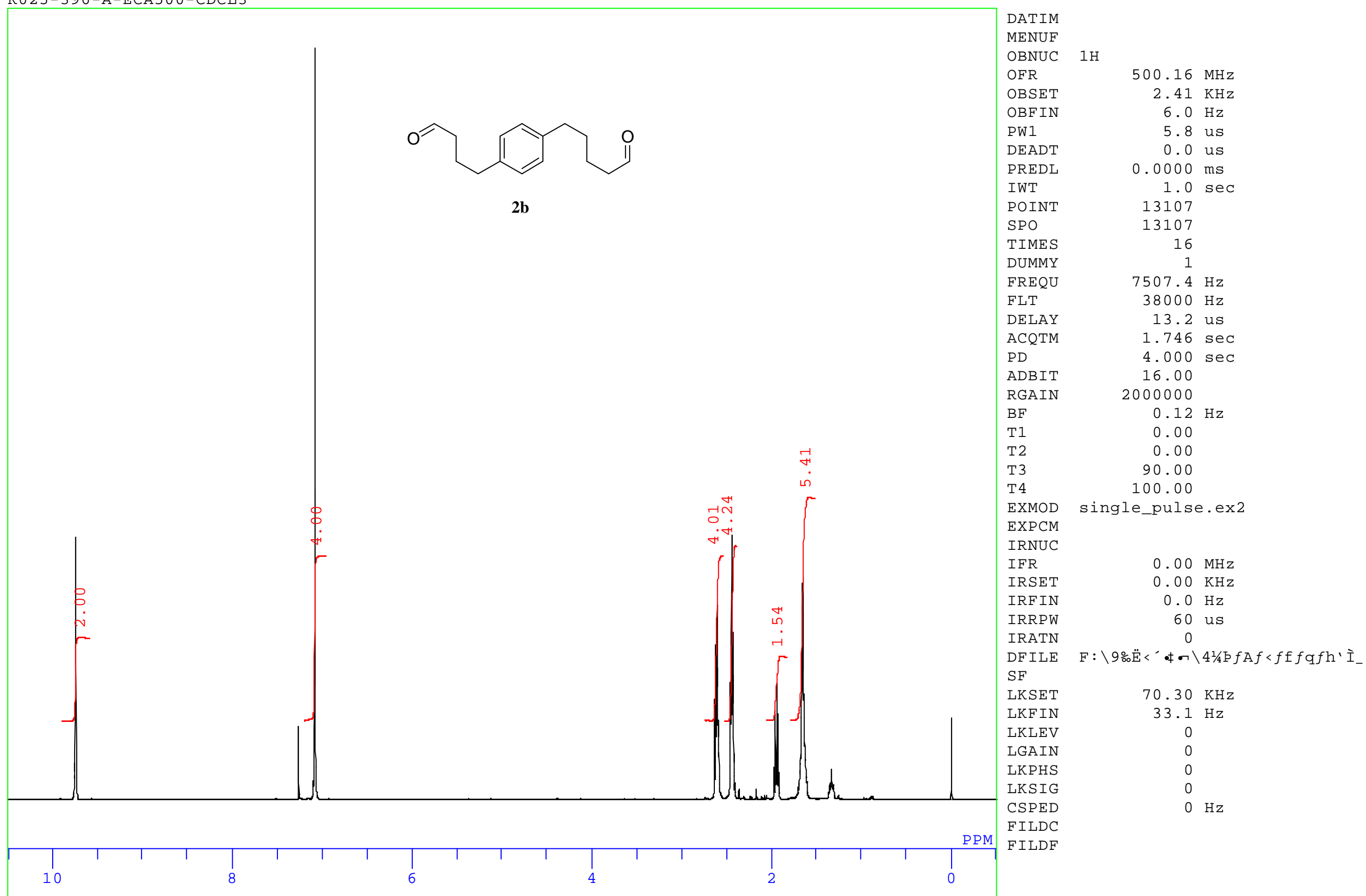


$F: \backslash 9 \% \ddot{E}<' \bullet \bullet \backslash 41 / 4 \mathrm{D} f \mathrm{~A} f<f f f q f h$ 'İ_CARBON .1 K025-396-A-ECA500-CDCL3

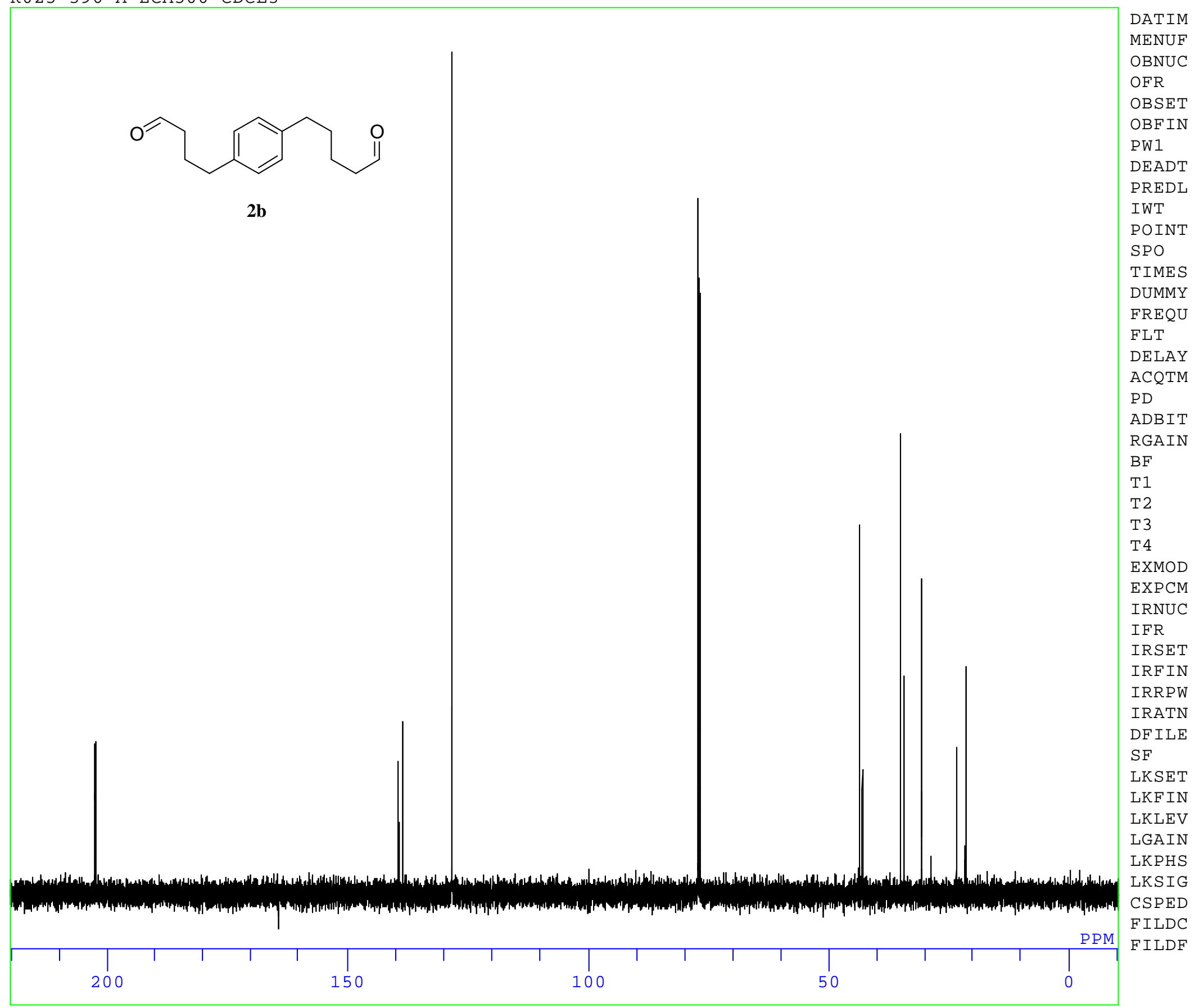


F : \EX.1-100\K021-18A1_PROTON.als

K021-18A1-400-CDCL3

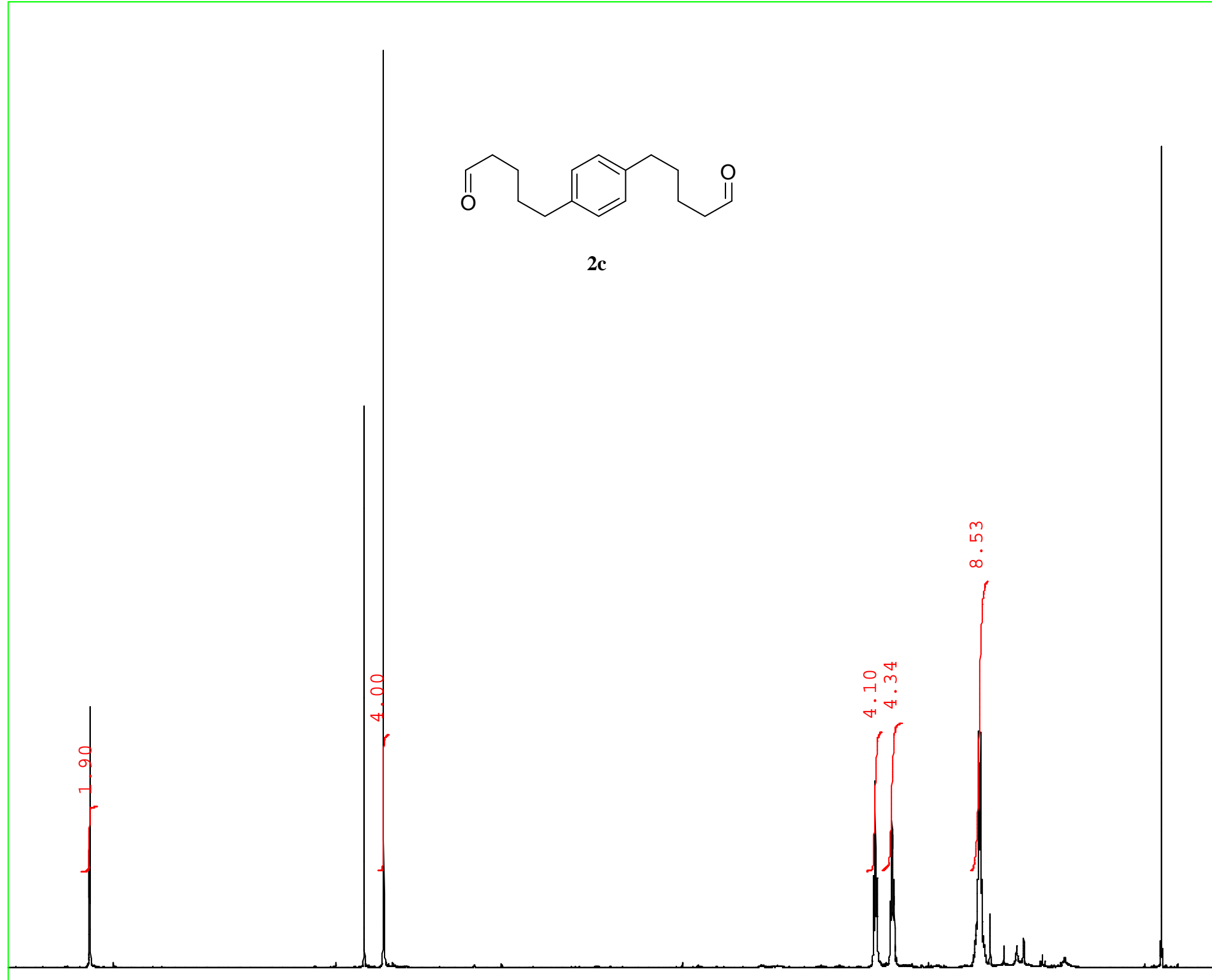

OBNUC

OBFIN

PW1

DEADT

PREDL

IWT

POINT

SPO

TIMES

DUMMY

FREQU

FLT

DEIAY

ACOTM

$\mathrm{PD}$

ADBIT

RGAIN

$B F$

$\mathrm{T} 1$

T2

T3

$\mathrm{T} 4$

EXMOD

EXPCM

IFR

IRSET

IRF IN

IRRPW

IRATN

DFILE

$\mathrm{SF}$

LKSET

LKFIN

LKLEV

LGAIN

KPHS

LKSIG

CSPED

FILDC

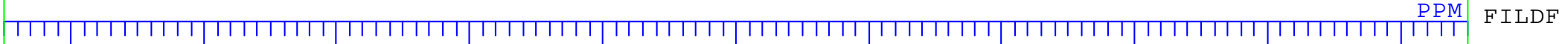

$4.19 \mathrm{KHz}$

$7.3 \mathrm{~Hz}$

5.7 us

0.0 us

$0.0000 \mathrm{~ms}$

$1.0 \mathrm{sec}$

10485

10485

16

$4801.8 \mathrm{~Hz}$

$31000 \mathrm{~Hz}$

16.1 us

$2.184 \mathrm{sec}$

$4.000 \mathrm{sec}$

16.00

200000

$0.12 \mathrm{~Hz}$

0.00

0.00

90.00

100.00

single_pulse.ex2

$0.00 \mathrm{MHz}$

$0.00 \mathrm{KHz}$

$0.0 \mathrm{~Hz}$

50 us

0

F : \Ex.1-100\K021-18A1_PROTOI

\section{$61.60 \mathrm{KHz}$}

$30.8 \mathrm{~Hz}$

0

0

0

$0 \mathrm{~Hz}$

10.0

9.0

8.0

7.0

6.0

5.0

4.0

3.0

2.0

1.0

0.0 
F $: \backslash \mathcal{V}, \mu$, ift $f H f<f \_\backslash d i a r d e h y d e \_C A R B O N . a l s$ diardehyde

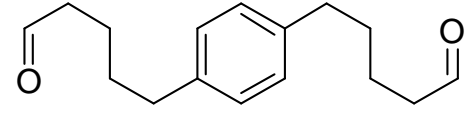

$2 \mathrm{c}$
DATIM

MENUF

OBNUC

OFR

OBSET

OBFIN

PW1

DEADT

PREDL

IWT

POINT

SPO

TIMES

DUMMY

FREQU

FLT

DELAY

ACOTM

$\mathrm{PD}$

ADBIT

RGAIN

$B F$

$\mathrm{T} 1$

$\mathrm{T} 2$

T 3

$\mathrm{T} 4$

EXMOD

EXPCM

IF $R$

IRSET

IRF IN

IRRPW

IRATN

DFILE

SF

LKSET

LKF IN

LKLEV

LGAIN

LKPHS

LKSIG

CSPED 
$\mathrm{F}: \backslash \mathrm{K} 006-[5+6]-\mathrm{dial}$ dehyde-1H. 1

KURO

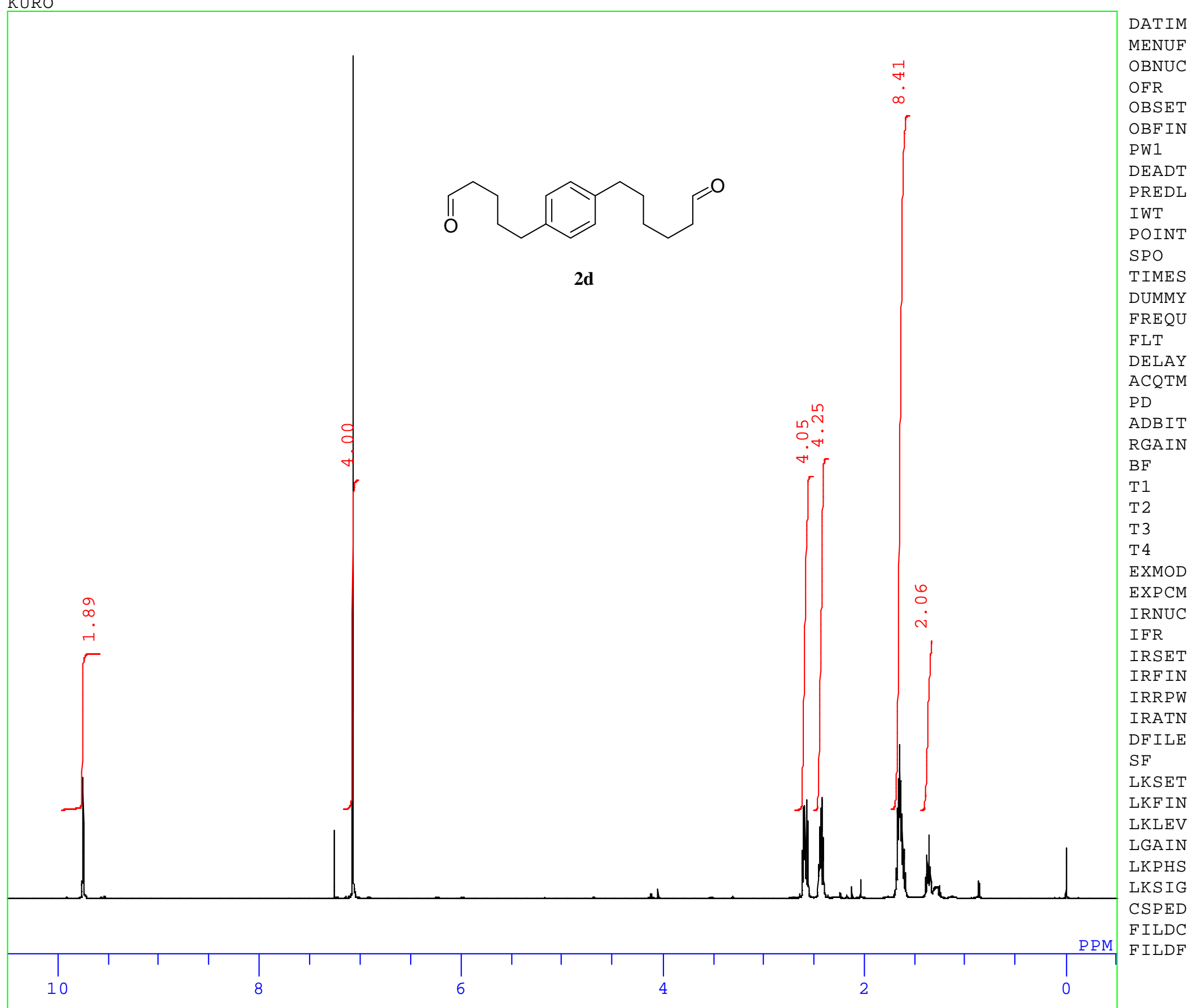

$1 \mathrm{H}$

OFR

DEADT

POIN

TIME

DUMMY

REQU

ACQTM

$\mathrm{PD}$

RGAIN

$\mathrm{BF}$

T1

$\mathrm{T} 4$

EXMOD

IRNUC

IFR

IRSET
IRF IN

IRRPW

RATN

DF I LE

LKSET

LKF IN

LKLEV

LKS IG

ILDC

$500.16 \mathrm{MHz}$

$2.41 \mathrm{KHz}$

$6.0 \mathrm{~Hz}$

5.8 us

0.0 us

$0.0000 \mathrm{~ms}$

$1.0 \mathrm{sec}$

13107

13107

16
1

$7507.4 \mathrm{~Hz}$

$38000 \mathrm{~Hz}$

13.2 us

$1.746 \mathrm{sec}$

$4.000 \mathrm{sec}$

16.00

2000000

$0.12 \mathrm{~Hz}$

0.00

0.00

90.00

100.00

single_pulse.ex2

$0.00 \mathrm{MHz}$

$0.00 \mathrm{KHz}$

$0.0 \mathrm{~Hz}$

90 us

F: \K006-[5+6]-dialdehyde-1H .

$70.30 \mathrm{KHz}$

$33.1 \mathrm{~Hz}$

0

0

0

$\mathrm{O} \mathrm{Hz}$ 
F: \K006_[5+6]-dialdehyde-_13C.1 KURO

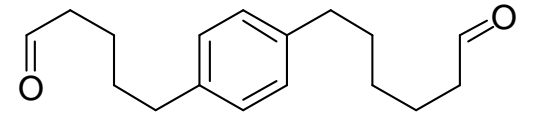

2d

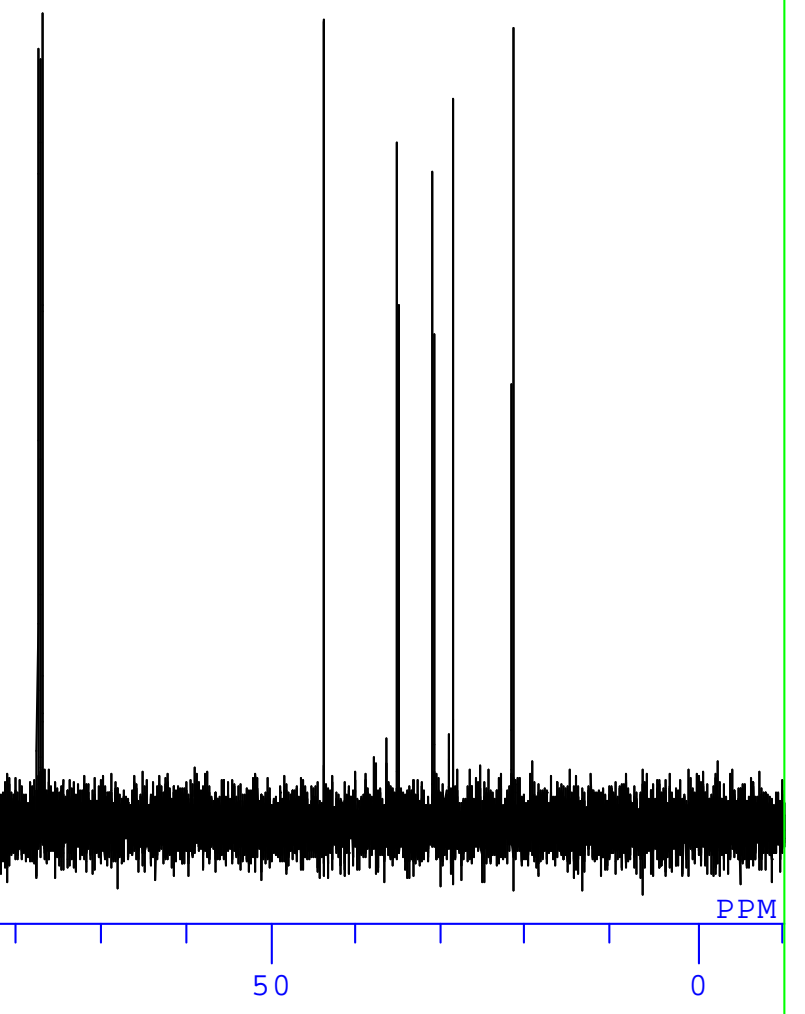

S31

DATIM

MENUF

OBNUC

OFR

OBSET

OBFIN

PW1

DEADT

PREDI

IWT

POINT

SPO

TIMES

DUMMY

FREQU

FLT

DELAY

ACOTM

PD

ADBIT

RGAIN

$\mathrm{BF}$

T 1

T2

EXMOD

EXPCM

IF $R$

IRSET

IRF IN

IRATN

DFILE SF

LKSET

LKFIN

LKLEV

LGAIN

KPHS

LKS IG

CSPED

FILDC

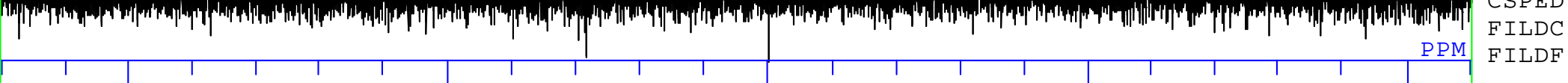

$125.77 \mathrm{MHz}$

$.87 \mathrm{KHz}$

$4.2 \mathrm{~Hz}$

3.8 us

0.0 us

$0.0000 \mathrm{~ms}$

$1.0 \mathrm{sec}$

26214

26214

31

$31446.1 \mathrm{~Hz}$ $158000 \mathrm{~Hz}$

20.7 us

$0.834 \mathrm{sec}$

$2.000 \mathrm{sec}$

16.00

2000000

$0.12 \mathrm{~Hz}$

0.00

0.00

90.00

100.00

single_pulse_dec

$0.00 \mathrm{MHz}$

$0.00 \mathrm{KHz}$

$0.0 \mathrm{~Hz}$

90 us

$\mathrm{F}: \backslash \mathrm{K} 006 \_[5+6]-\mathrm{dialdehyde- \_ 13}$

$70.30 \mathrm{KHz}$

$33.1 \mathrm{~Hz}$

0

0

0

$\mathrm{OHz}$ 
$\mathrm{F}: \backslash 12 \% \ddot{E}<‘ \bullet \bullet \backslash \mathrm{K} 025-400-\mathrm{A} \_P R O T O N .1$

K025-400-A-ECA500-DCDL3

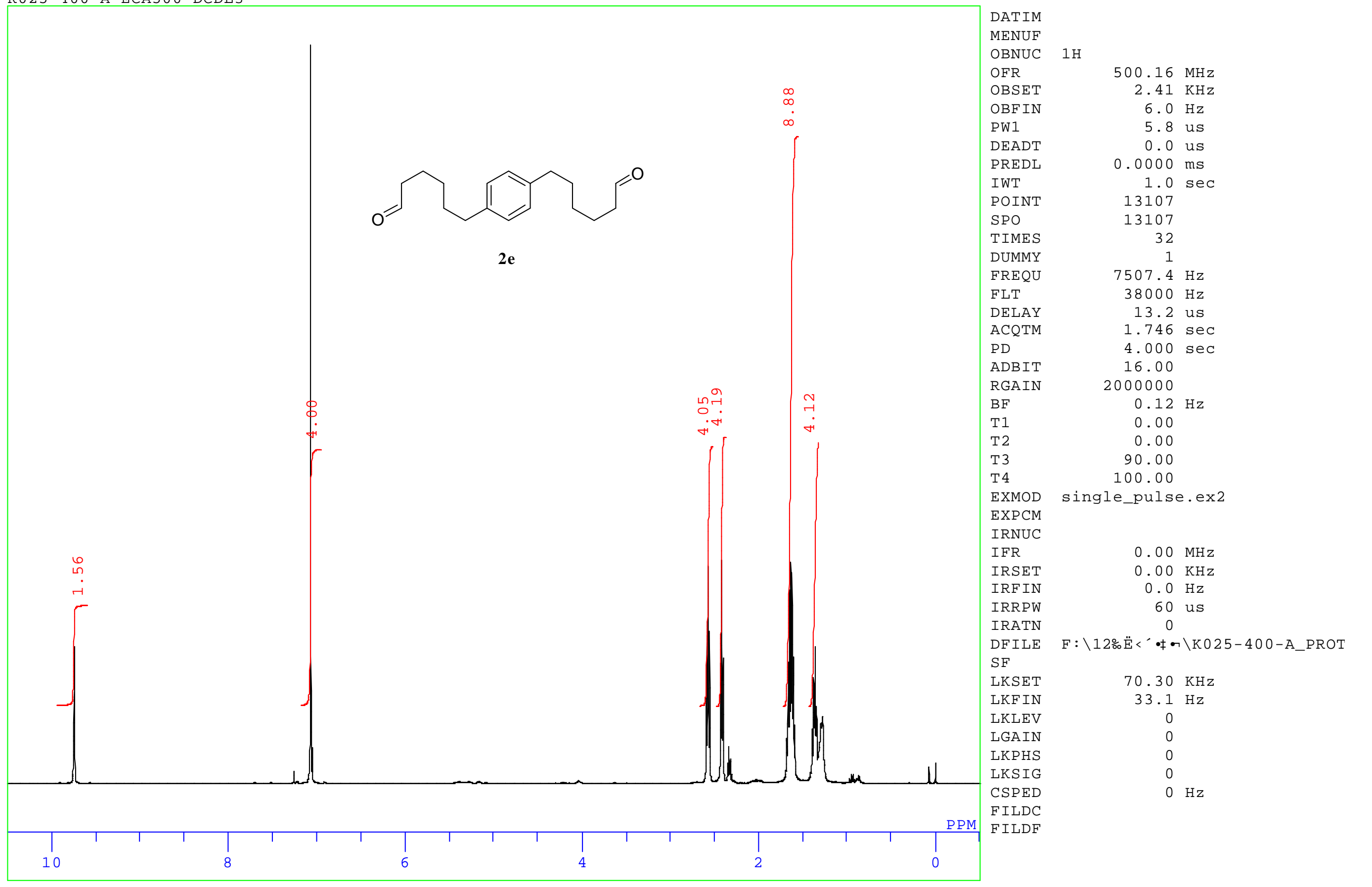


$\mathrm{F}: \backslash 12 \% \ddot{E}<\cdot \bullet \bullet \backslash \mathrm{K} 025-400-\mathrm{A} \_C A R B O N .1$

K025-400-A-ECA 500-DCDL3

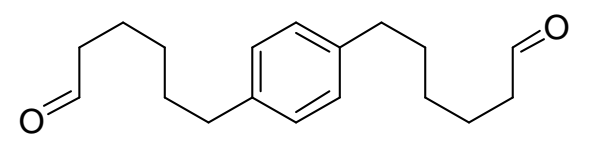

$2 \mathbf{e}$
DATIM

MENUF

OBNUC

OFR

OBSET

OBEIN

PW1

DEADT

PREDL

IWT

POINT

SPO

TIMES

DUMMY

FREQU

FLT

DELAY

ACOTM

$\mathrm{PD}$

ADBIT

RGAIN

$B F$

$\mathrm{T} 1$

T2

T3

T 4

EXMOD

EXPCM

IFR

IRSET

IRFIN

IRRPW

IRATN

DEILE

$\mathrm{SF}$

LKSET

LKF IN

LKLEV

LGAIN

LKP HS

LKSIG

CSPED

FILDC

PPM FILDF
$13 c$

$$
125.77 \mathrm{MHz}
$$

$7.87 \mathrm{KHz}$

$4.2 \mathrm{~Hz}$

4.1 us

0.0 us

$0.0000 \mathrm{~ms}$

$1.0 \mathrm{sec}$

26214

26214

128

$31446.1 \mathrm{~Hz}$

$158000 \mathrm{~Hz}$

20.7 us

$0.834 \mathrm{sec}$

$4.000 \mathrm{sec}$

16.00

2000000

$0.12 \mathrm{~Hz}$

0.00

0.00

90.00

100.00

single_pulse_dec

$0.00 \mathrm{MHz}$

$0.00 \mathrm{KHz}$

$0.0 \mathrm{~Hz}$

60 us

0

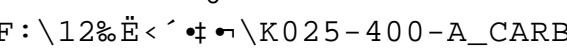

$70.30 \mathrm{KHz}$

$33.1 \mathrm{~Hz}$

0

0

0

$\mathrm{OHz}$

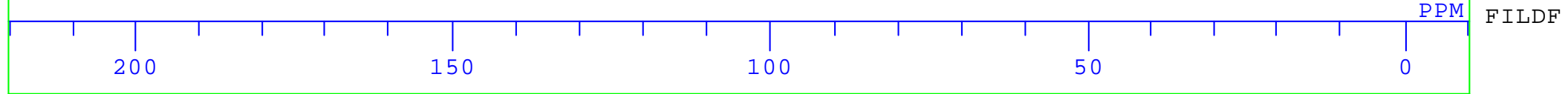




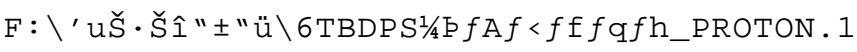

K025-380-A-ECA 500-CDCL3

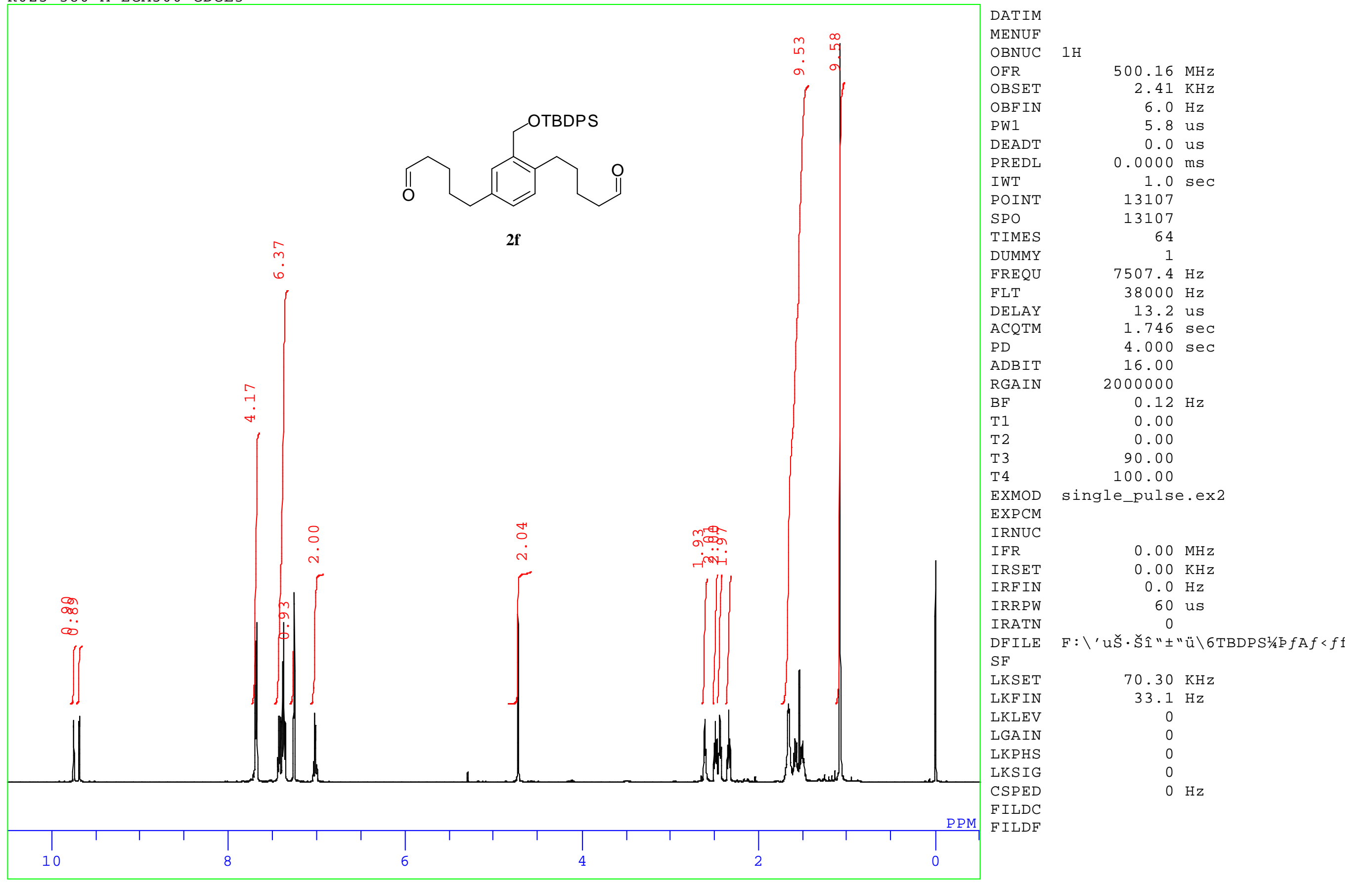


$F: \backslash$ ' uŠ $\cdot \check{S} \hat{I} " \pm$ "ü $\backslash 6 \mathrm{TBDPS} S^{1 / 4} \mathrm{P} f \mathrm{~A} f<f f f q f h \_C A R B O N .1$ K025-380-A-ECA 500-CDCL3

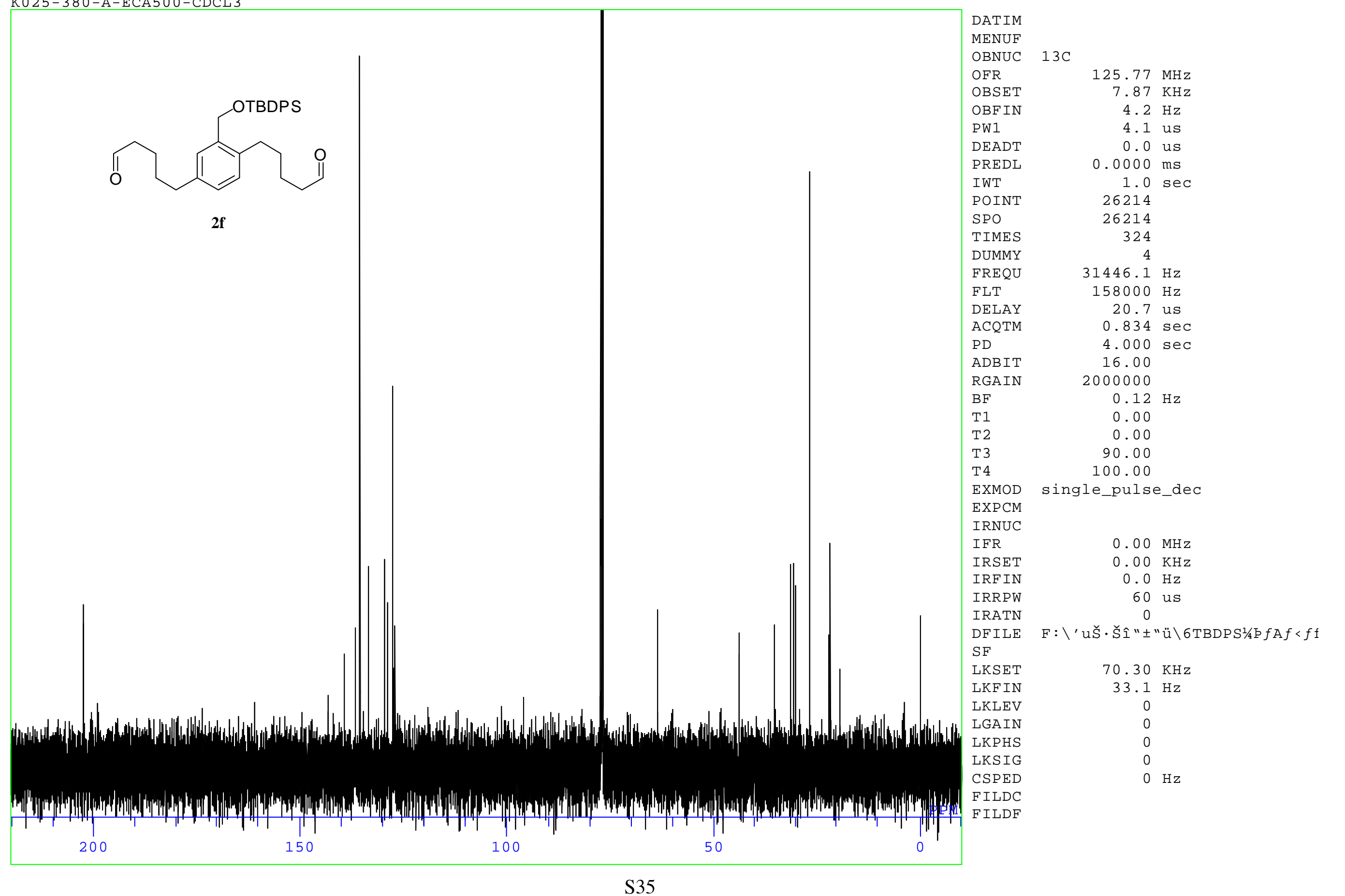




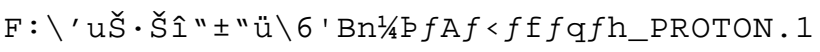

K025-393-A-ECA 500-CDCL3

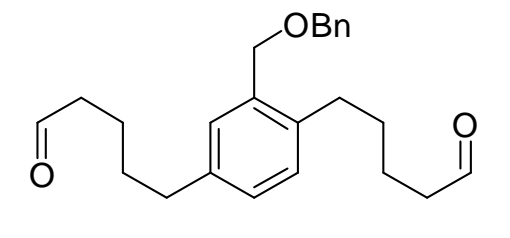

$2 \mathrm{~g}$
|

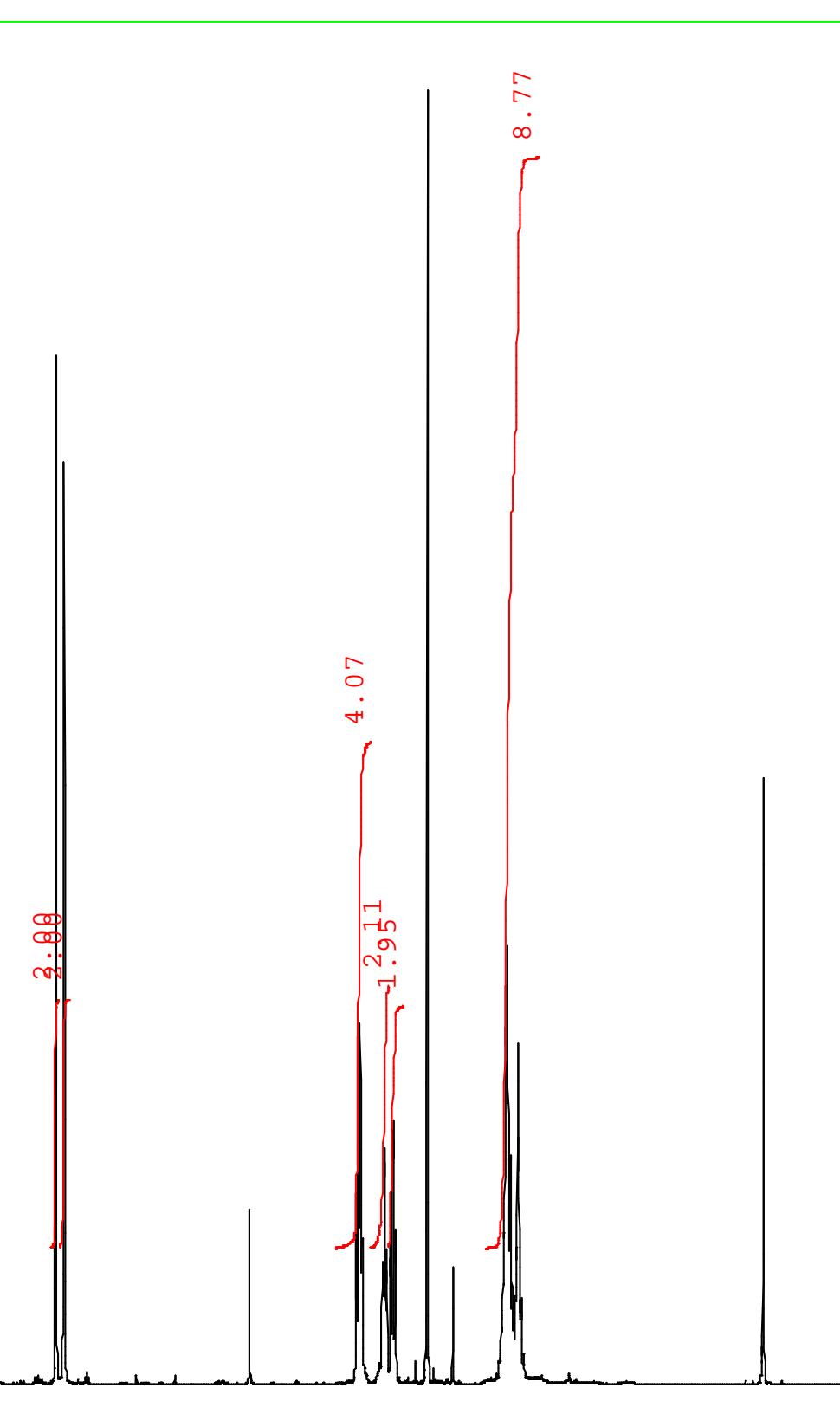

MENUF

OBNUC $1 \mathrm{H}$

OFR

OBSET

OBFIN

PW1

DEADT

PREDL

IWT

POINT

SPO

TIMES

DUMMY

FREQU

FLT

DELAY

ACOTM

$\mathrm{PD}$

ADBIT

RGAIN

$\mathrm{BF}$

$\mathrm{T} 1$

T2

EXMOD

EXPCM

IRNUC

IFR

IRSET

IRFIN

IRATN

DF ILE $\mathrm{SF}$

IKSET

LKFIN

LKLEV

LGAIN

LKS I G

CSPED

FILDC

PPM FILDF

$500.16 \mathrm{MHz}$ $2.41 \mathrm{KHz}$

$6.0 \mathrm{~Hz}$

5.8 us

0.0 us

$0.0000 \mathrm{~ms}$

$1.0 \mathrm{sec}$

13107

13107

64

$7507.4 \mathrm{~Hz}$

$38000 \mathrm{~Hz}$

13.2 us

$1.746 \mathrm{sec}$

$4.000 \mathrm{sec}$

16.00

2000000

$0.12 \mathrm{~Hz}$

0.00

0.00

90.00

100.00

single_pulse.ex2

$0.00 \mathrm{MHz}$

$0.00 \mathrm{KHz}$

$0.0 \mathrm{~Hz}$

60 us

$: \backslash^{\prime} u \check{S} \cdot \check{S} \hat{I} " \pm$ "ü $\backslash \sigma^{\prime} B n^{1 / 4} \mathrm{E} f A f<f f f c$

$70.30 \mathrm{KHz}$

$33.1 \mathrm{~Hz}$

0

0

0

$\mathrm{OHz}$ 


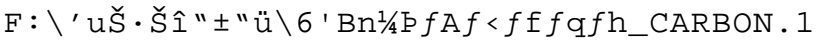

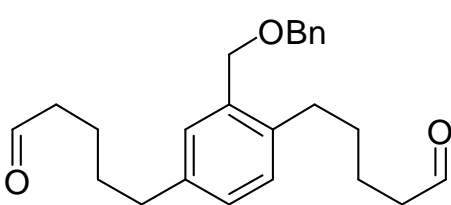

$2 \mathrm{~g}$

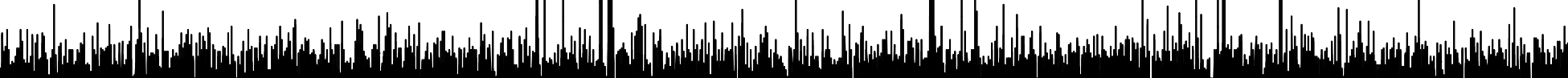

DATIM

MENUF

OBNUC

OFR

OBSET

OBFIN

PW1

DEADT

PREDI

POIN

SPO

TIMES

DUMMY

FREQU

FLT

DELAY

ACOTM

$\mathrm{PD}$

ADBIT

RGAIN

$\mathrm{BF}$

$\mathrm{T} 1$

T2

T4

EXMOD

EXPCM

IFR

IRSET

IRF IN

IRRPW

IRATN

DFILE $S F$

LKFIN

LKLEV

LGAIN

LKPHS

LKS I G

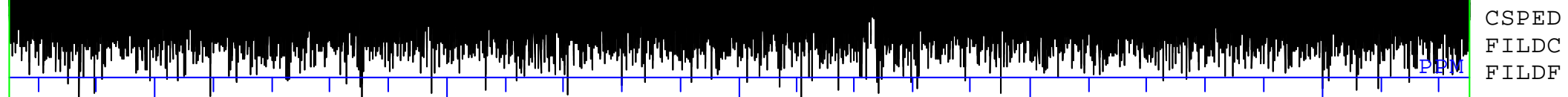

150
$3 c$

$125.77 \mathrm{MHz}$

$.87 \mathrm{KHz}$

$4.2 \mathrm{~Hz}$

4.1 us

0.0 us

$0.0000 \mathrm{~ms}$

$1.0 \mathrm{sec}$

26214

26214

256

$31446.1 \mathrm{~Hz}$

$158000 \mathrm{~Hz}$

20.7 us

$0.834 \mathrm{sec}$

$4.000 \mathrm{sec}$

16.00

2000000

$0.12 \mathrm{~Hz}$

0.00

0.00

90.00

100.00

single_pulse_dec

$0.00 \mathrm{MHz}$

$0.00 \mathrm{KHz}$

$0.0 \mathrm{~Hz}$

$60 \mathrm{us}$

$F: \backslash$ ' uŠ

$70.30 \mathrm{KHz}$

$33.1 \mathrm{~Hz}$

0

0

$\mathrm{O} \mathrm{Hz}$ 


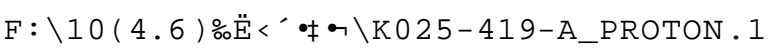

K025-419-A-ECA500-CDCL3

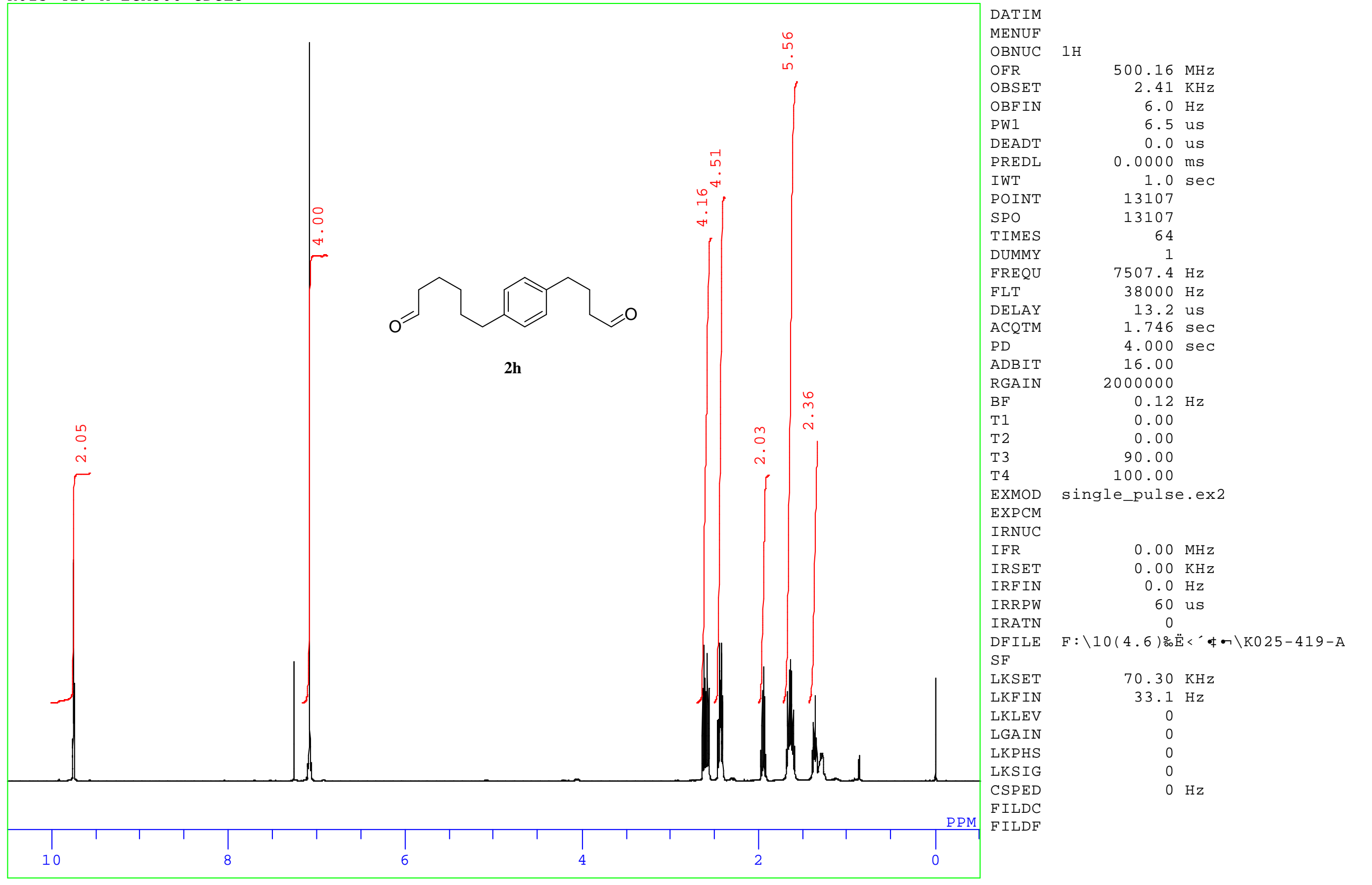


$\mathrm{F}: \backslash 10(4.6) \% \ddot{\mathrm{E}}<\bullet \bullet \backslash \mathrm{K} 025-419-\mathrm{A}$ _CARBON .1

K025-419-A-ECA500-CDCL3

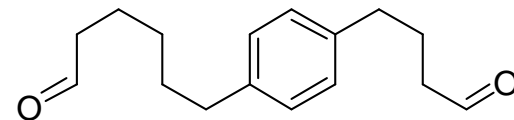

$2 h$
DAT IM

MENUF

OBNUC

OFR

OBSET

OBFIN

PW1

DEADT

PREDI

IWT

POIN

SPO

TIMES

DUMMY

FREQU

FLT

DELAY

ACOTM

$P D$

ADBIT

RGAIN

$\mathrm{BF}$

$\mathrm{T} 1$

T2

T 4

EXMOD

EXPCM

IFR

IRSET

IRF IN

IRRPW

IRATN

DF I LE

SF

LKSET

LKF IN

LKLEV

LGAIN

LKSIG

CSPED

FILDC

PPM FILDF
$3 c$

$125.77 \mathrm{MHz}$

$7.87 \mathrm{KHz}$

$4.2 \mathrm{~Hz}$

4.3 us

0.0 us

$0.0000 \mathrm{~ms}$

$1.0 \mathrm{sec}$

26214

26214

256

$31446.1 \mathrm{~Hz}$

$158000 \mathrm{~Hz}$

20.7 us

$0.834 \mathrm{sec}$

$4.000 \mathrm{sec}$

16.00

2000000

$0.12 \mathrm{~Hz}$

0.00

0.00

$$
90.00
$$$$
100.00
$$

single_pulse_dec

$0.00 \mathrm{MHz}$

$0.00 \mathrm{KHz}$

$0.0 \mathrm{~Hz}$

60 us

0

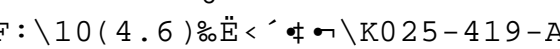

$70.30 \mathrm{KHz}$

$33.1 \mathrm{~Hz}$

0

0

0

$\mathrm{OHz}$

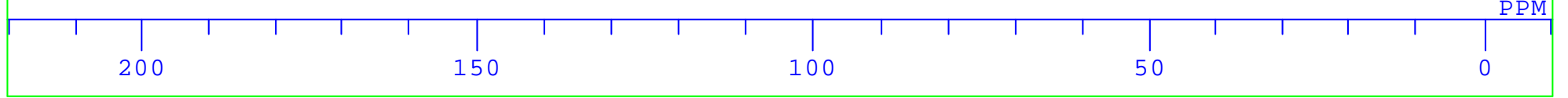


$F: \backslash \mathbb{E}^{3} f f \bullet\left[f^{\wedge} \backslash K 025-500-A \_P R O T O N .1\right.$

K025-500-A-ECA 500-CDCL3

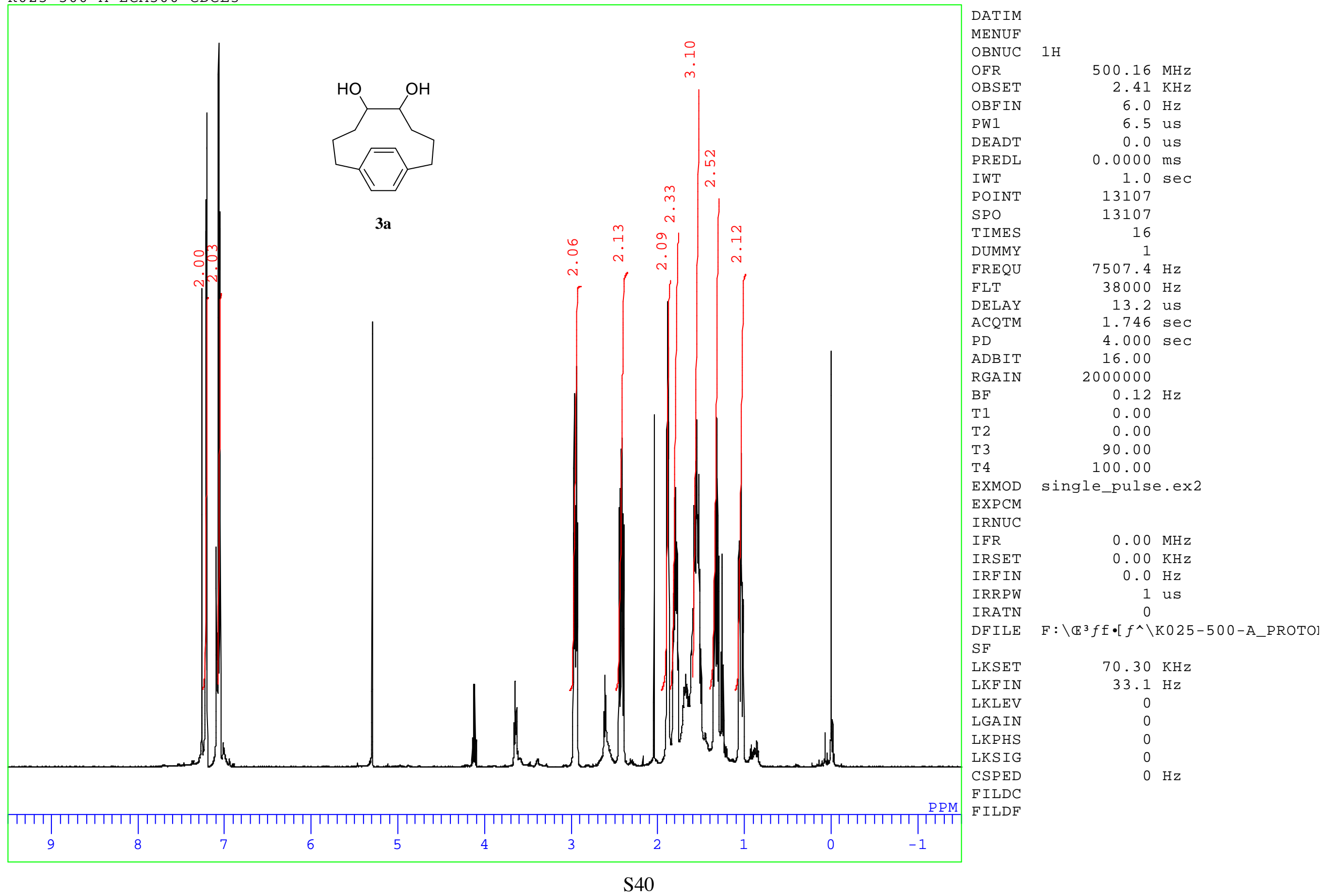




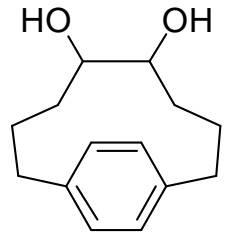

3a
DATIM

MENUF

OBNUC

OFR

OBSET

OBFIN

PW1

DEADT

PREDL

IWT

POINT

SPO

TIMES

DUMMY

FREQU

FLT

DELAY

ACOTM

$\mathrm{PD}$

ADBIT

RGAIN

$B F$

$\mathrm{T} 1$

T2

T3

$\mathrm{T} 4$

EXMOD
EXPCM

EXPCM

IFR

IRSET

IRF IN

IRRPW

IRATN

DF ILE $\mathrm{SF}$

LKSET

LKFIN

LKLEV

LGAIN

LKPHS

LKSIG

CSPED

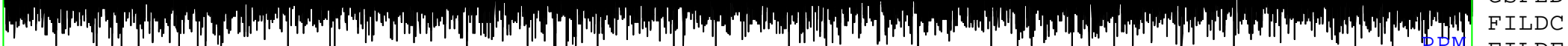

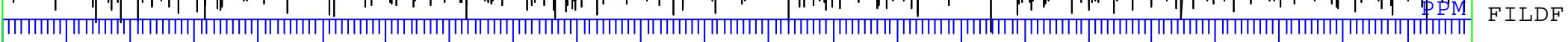

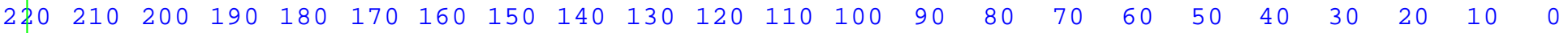

$13 \mathrm{C}$

$125.77 \mathrm{MHz}$

$.87 \mathrm{KHz}$

$4.2 \mathrm{~Hz}$

4.0 us

0.0 us

$0.0000 \mathrm{~ms}$

$1.0 \mathrm{sec}$

26214

26214

24

$31446.1 \mathrm{~Hz}$

$158000 \mathrm{~Hz}$

20.7 us

$0.834 \mathrm{sec}$

$4.000 \mathrm{sec}$

16.00

2000000

$0.12 \mathrm{~Hz}$

0.00

0.00

90.00

100.00

single_pulse_dec

$0.00 \mathrm{MHz}$

$0.00 \mathrm{KHz}$

$0.0 \mathrm{~Hz}$

$F: \backslash \mathbb{E}^{3} f f \bullet\left[f^{\wedge} \backslash K 025-500-A \_C A R B O\right]$

$$
\begin{array}{rl}
70.30 & \mathrm{KHz} \\
33.1 & \mathrm{~Hz} \\
0 & \\
0 & \\
0 & \\
0 & \\
0 & \mathrm{~Hz}
\end{array}
$$


F: \K006_[9] paracyclophane-diol-1H.1

MURASAKI

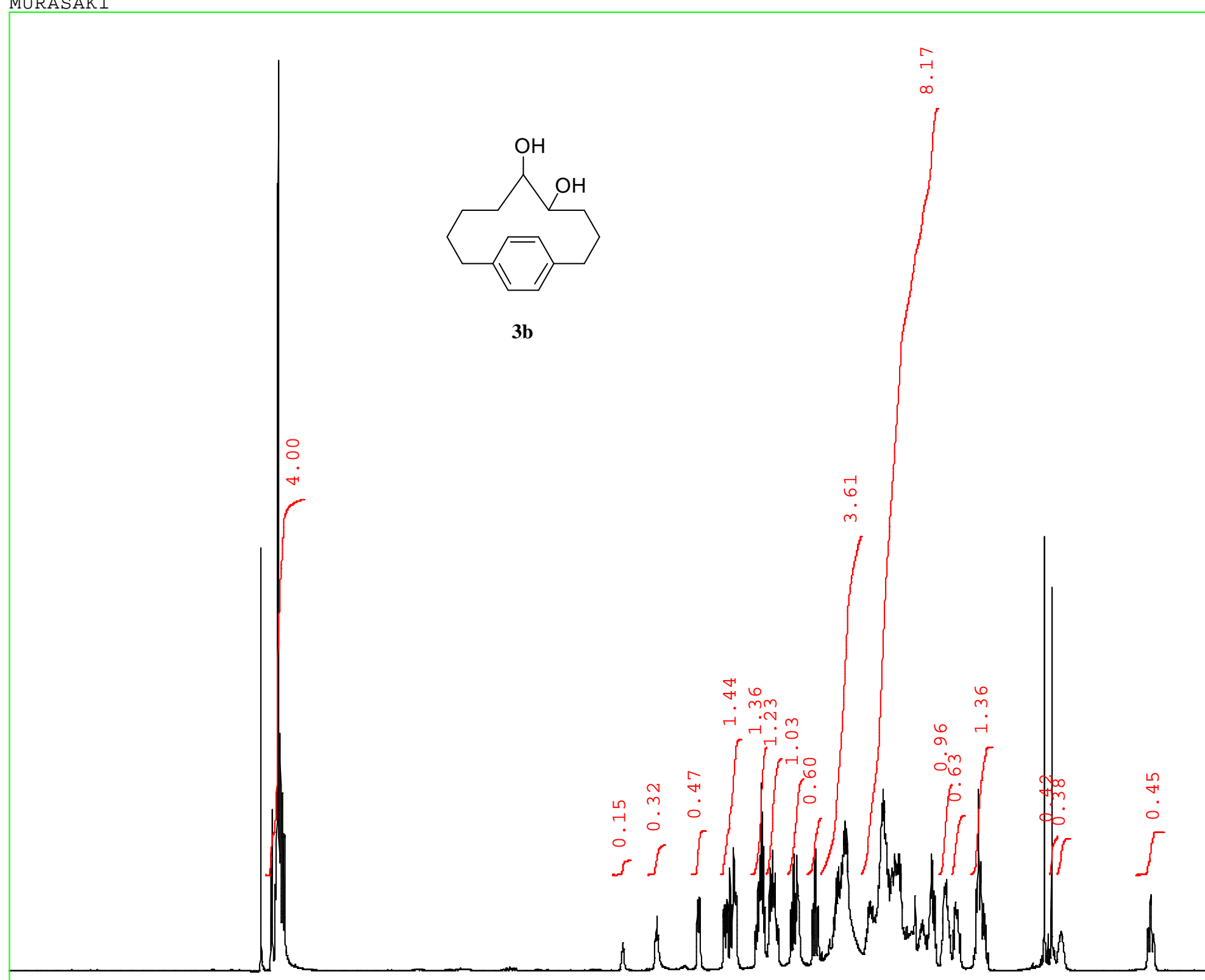

DATIM

MENUF

OBNUC

OFR

OBSET

OBFIN

PW1

DEADT

PREDL

IWT

POINT

SPO

TIMES

DUMMY

FREQU

FLT

DELAY

ACOTM

PD

ADBIT

RGAIN

$\mathrm{BF}$

T 1

T2

EXMOD

EXPCM

RNUC

IFR

IRSET

IRE IN

I RRPW

IRATN

DFILE

SF

LKSET

LKF IN

LKLEV

LGAIN

LKS I G

CSPED

FILDC
$500.16 \mathrm{MHz}$ $2.41 \mathrm{KHz}$

$6.0 \mathrm{~Hz}$

5.8 us

0.0 us

$0.0000 \mathrm{~ms}$

$1.0 \mathrm{sec}$

13107

13107

16

$7507.4 \mathrm{~Hz}$

$38000 \mathrm{~Hz}$

13.2 us

$1.746 \mathrm{sec}$

$4.000 \mathrm{sec}$

16.00

2000000

$0.12 \mathrm{~Hz}$

0.00

0.00

90.00

100.00

single_pulse.ex2

$0.00 \mathrm{MHz}$

$0.00 \mathrm{KHz}$

$0.0 \mathrm{~Hz}$

90 us

F : \K006_[9] paracyclophane-dj

$70.30 \mathrm{KHz}$

$33.1 \mathrm{~Hz}$

0

0

0

$\mathrm{O} \mathrm{Hz}$

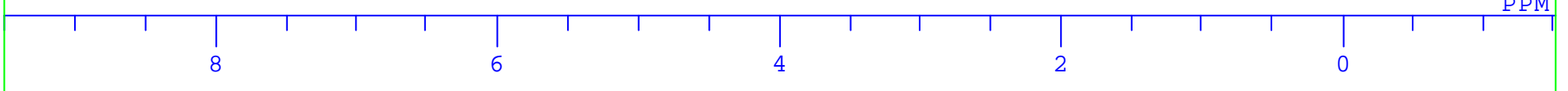


F: \K006_[9] paracyclophane-diol-13C.1 MURASAKI

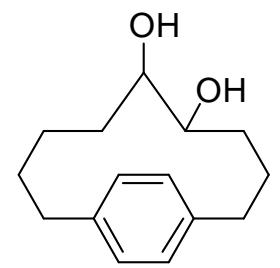

$3 b$

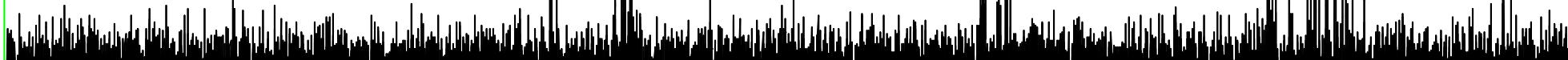

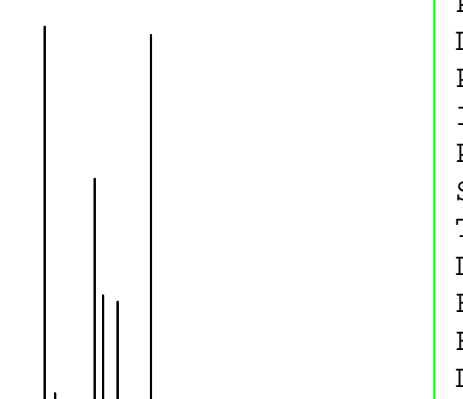

DELAY

ACQTM

ADBIT

RGAIN

$\mathrm{BF}$

T1

T2

T 3

T 4

EXMOD

EXPCM

IFR

IRSET

IRFIN

IRRPW

IRATN

DFILE $\mathrm{SF}$

LKSET

LKF IN

LKLEV

LGAIN

LKPHS

CSPED

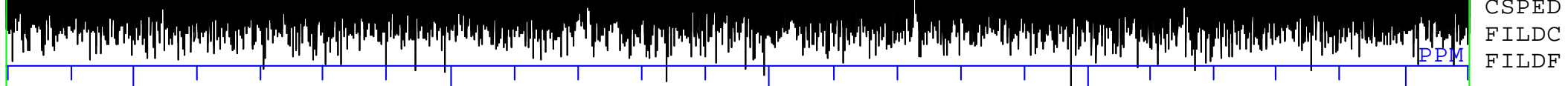

150

100
$13 \mathrm{C}$

$125.77 \mathrm{MHz}$

$7.87 \mathrm{KHz}$

$4.2 \mathrm{~Hz}$

3.8 us

0.0 us

$0.0000 \mathrm{~ms}$

$1.0 \mathrm{sec}$

26214

26214

325

$31446.1 \mathrm{~Hz}$

$158000 \mathrm{~Hz}$

20.7 us

$0.834 \mathrm{sec}$

$2.000 \mathrm{sec}$

16.00

2000000

$0.12 \mathrm{~Hz}$

0.00

0.00

90.00

100.00

single_pulse_dec

$0.00 \mathrm{MHz}$

$0.00 \mathrm{KHz}$

$0.0 \mathrm{~Hz}$

F : \K006_[9] paracyclophane-dj

$70.30 \mathrm{KHz}$

$33.1 \mathrm{~Hz}$

0

0

0

$\mathrm{OHz}$ 
F : $\backslash$,,$\mu$, cft $f H f<f \_\backslash[10]$ paracyclophanediol_PROTON.als [10] paracyclophanediol

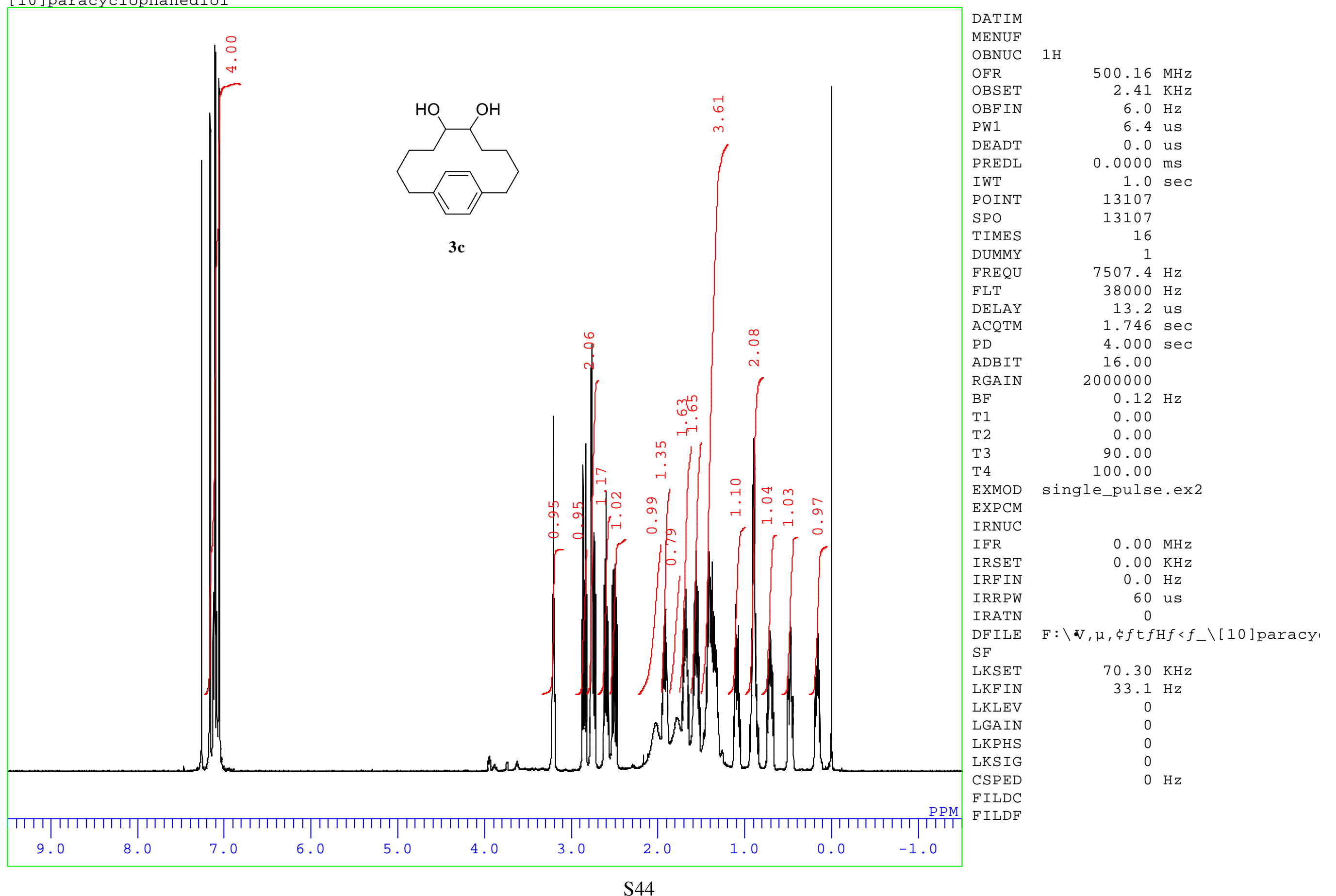


$F: \backslash \bullet V, \mu$, cft $f H f<f \_\backslash[10]$ paracyclophanediol_CARBON.als [10] paracyclophanedio]

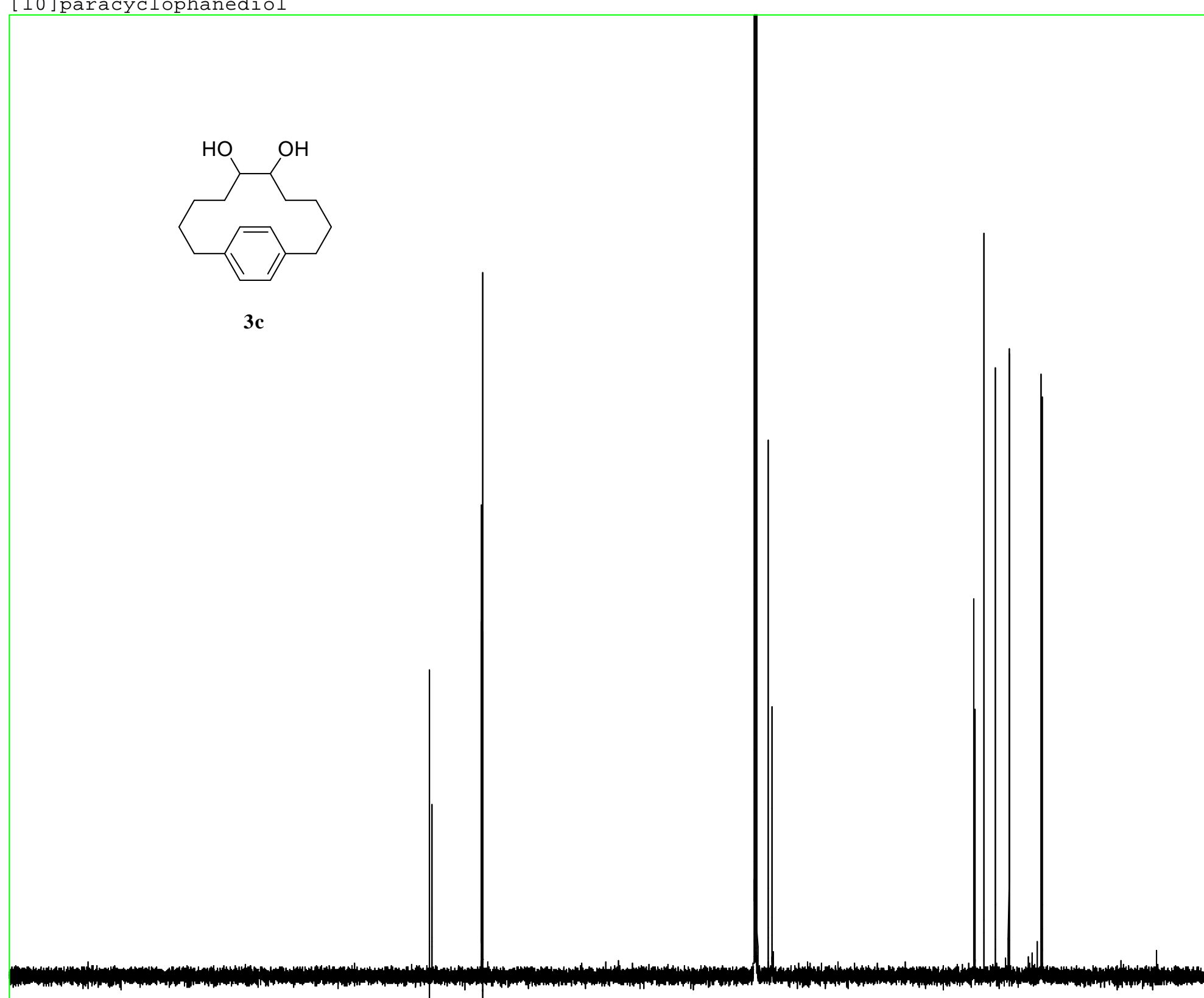

OBNUC

OFR

OBSET

OBFIN

$\mathrm{PW} 1$

DEADT

PREDL

IWT

POINT

SPO

TIMES

DUMMY

FREQU

FLT

DELAY

ACOTM

$\mathrm{PD}$

ADBIT

RGAIN

T 1

T2

T3

T 4

EXMOD

EXP CM

I RNUC

IF R

IRSET

IRE IN

IRRPW

IRATN

DEILE

$\mathrm{SF}$

LKSET

LKF IN

LKLEV

LGAIN

LKPHS

LKS IG

CSPED

FILDC

$125.77 \mathrm{MHz}$

$7.87 \mathrm{KHz}$

$4.2 \mathrm{~Hz}$

4.1 us

0.0 us

$0.0000 \mathrm{~ms}$

$1.0 \mathrm{sec}$

26214

26214

1536

$31446.1 \mathrm{~Hz}$

$158000 \mathrm{~Hz}$

20.7 us

$0.834 \mathrm{sec}$

$4.000 \mathrm{sec}$

16.00

2000000

$0.12 \mathrm{~Hz}$

0.00

0.00

90.00

100.00

single pulse dec

$$
\begin{array}{rl}
0.00 & \mathrm{MHz} \\
0.00 & \mathrm{KHz} \\
0.0 & \mathrm{~Hz} \\
60 & \mathrm{us} \\
0 &
\end{array}
$$

$F: \backslash \bullet, \mu, \xi f t f H f<f_{-} \backslash[10]$ paracy

$$
70.30 \mathrm{KHz}
$$$$
33.1 \mathrm{~Hz}
$$

0

0

0 


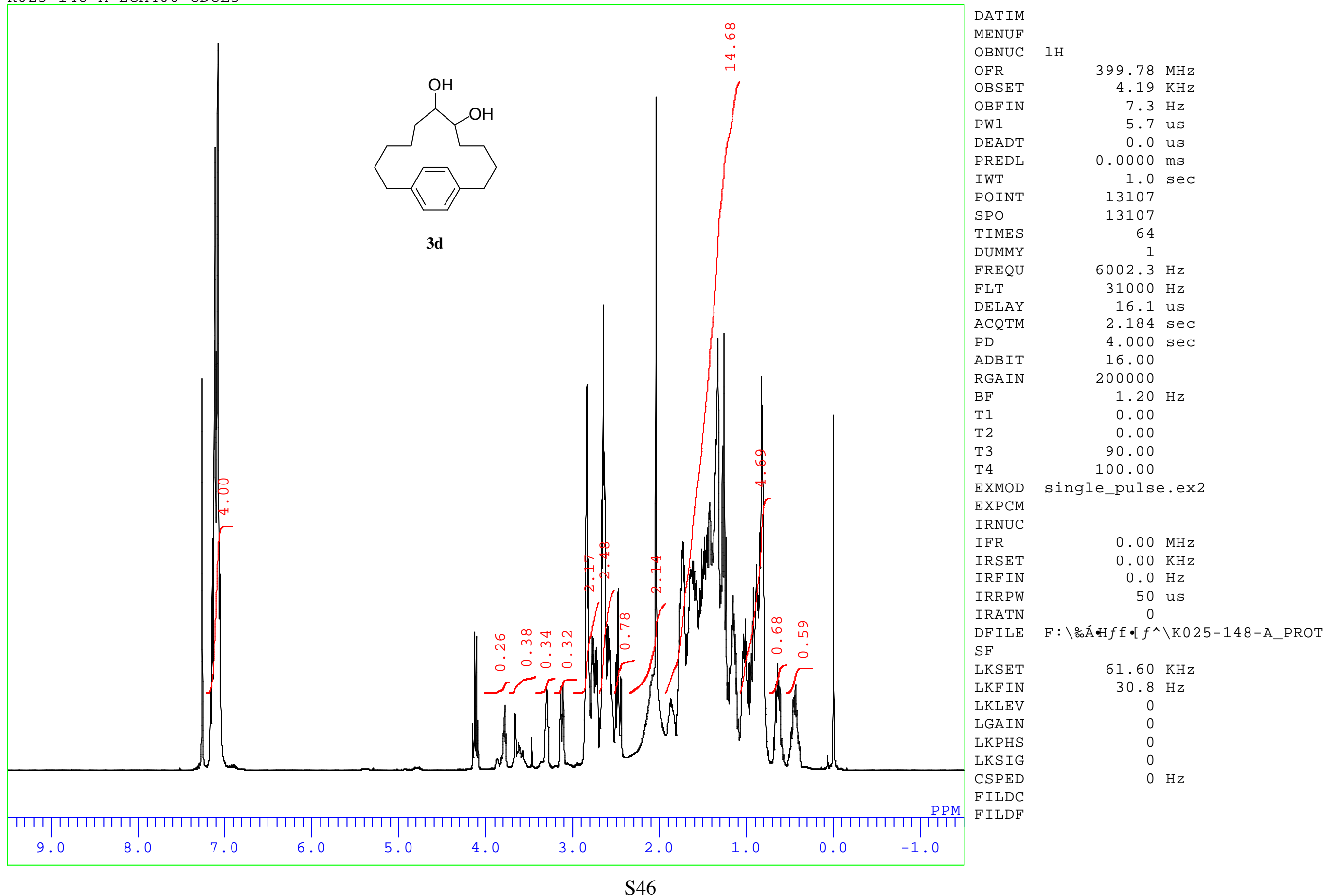




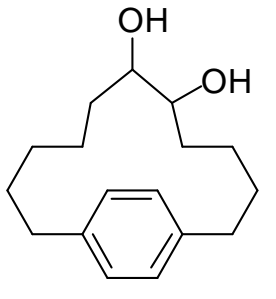

3d
DATIM

MENUF

OBNUC

OFR

OBSET

OBFIN

PW1

DEADT

PREDL

IWT

POINT

SPO

TIMES

DUMMY

FREQU

FLT

DELAY

ACOTM

$\mathrm{PD}$

ADBIT

RGAIN

$B F$

$\mathrm{T} 1$

$\mathrm{T} 2$

T3

$\mathrm{T} 4$

EXMOD

EXPCM

IFR

IRSET

IRF IN

IRRPW

IRATN

DFILE

$\mathrm{SF}$

LKSET

LKF IN

LKLEV

LGAIN

LKPHS

LKSIG

CSPED FILDC FILDF
$13 c$

$100.53 \mathrm{MHz}$ $5.35 \mathrm{KHz}$

$5.9 \mathrm{~Hz}$

3.3 us

0.0 us

$0.0000 \mathrm{~ms}$

$1.0 \mathrm{sec}$

26214

26214

215

$25125.2 \mathrm{~Hz}$

$126000 \mathrm{~Hz}$

21.9 us

$1.043 \mathrm{sec}$

2.000 sec

16.00

150000

$1.20 \mathrm{~Hz}$

0.00

0.00

$$
90.00
$$

100.00

single_pulse_dec

$0.00 \mathrm{MHz}$

$0.00 \mathrm{KHz}$

$0.0 \mathrm{~Hz}$

$\mathrm{F}: \backslash \stackrel{\circ}{\circ} \dot{A} \bullet \mathrm{H} f \bullet\left[f^{\wedge} \backslash \mathrm{K} 025-148-\mathrm{A} \_\mathrm{CARB}\right.$

$$
\begin{array}{rl}
61.60 & \mathrm{KHz} \\
30.8 & \mathrm{~Hz} \\
0 & \\
0 & \\
0 & \\
0 & \\
0 & \mathrm{~Hz}
\end{array}
$$


F: \K006_[12] paracyclophane-diol-1H.1 MIDORI

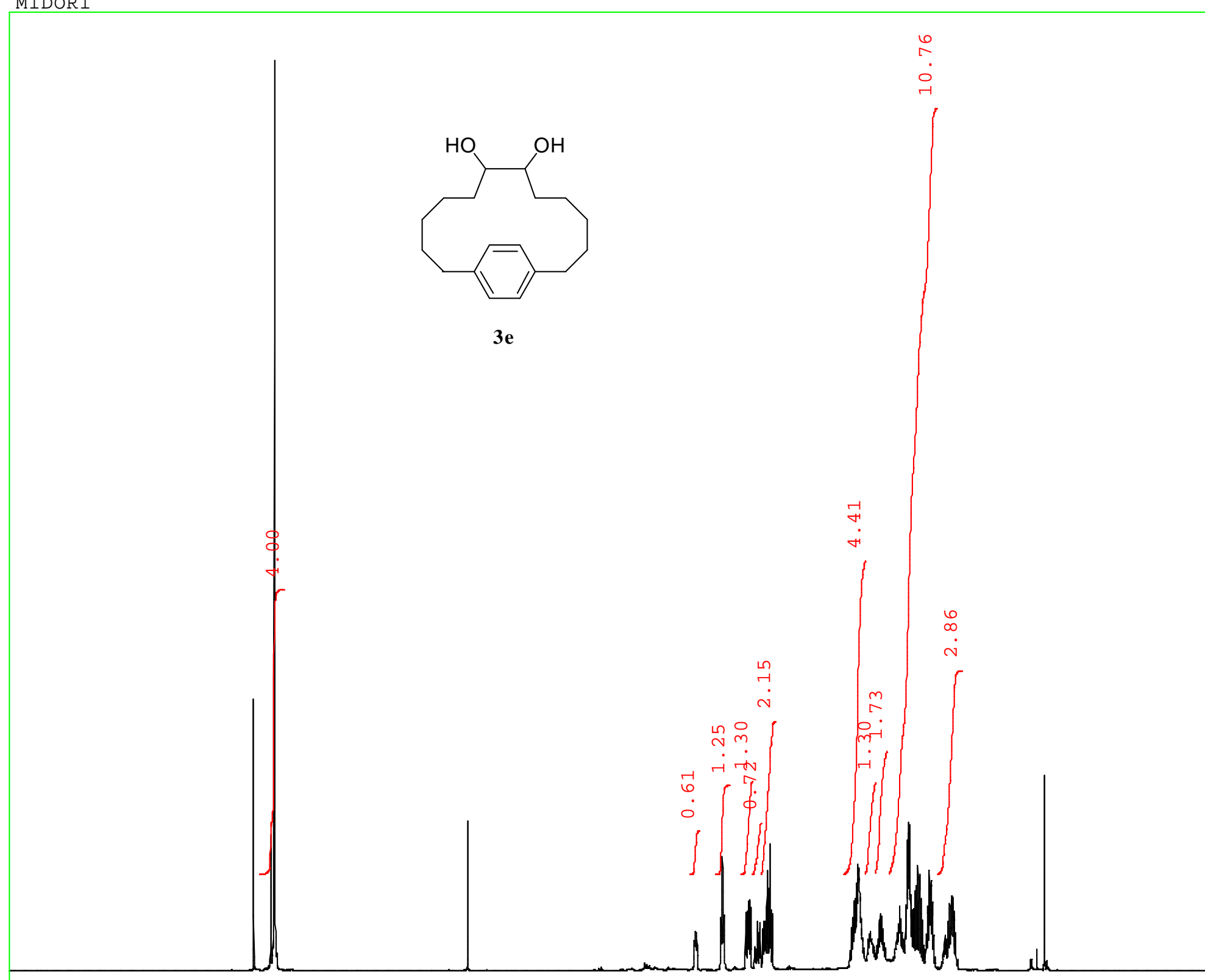

DAT IM

MENUF

OBNUC

OFR

BSET

OBFIN

PW1

DEADT

RED

IWT

POIN

SPO

TIMES

DUMMY

FREQU

FLT

DELAY

ACOTM

PD

ADBIT

RGAIN

$\mathrm{BF}$

T 1

$\mathrm{T} 2$

EXMOD

EXPCM

IRNUC

IFR

IRSET

IRF IN

DEILE

$S F$

LKSET

LKFIN

LKLEV

LGAIN

LKPHS

LKSIG

CSPED

FILDC

PPM FILDF
$500.16 \mathrm{MHz}$ $2.41 \mathrm{KHz}$

$6.0 \mathrm{~Hz}$

5.8 us

0.0 us

$0.0000 \mathrm{~ms}$

$1.0 \mathrm{sec}$

13107

13107

16

$7507.4 \mathrm{~Hz}$ $38000 \mathrm{~Hz}$

13.2 us

$1.746 \mathrm{sec}$

$4.000 \mathrm{sec}$

16.00

2000000

$0.12 \mathrm{~Hz}$

0.00

0.00

90.00

100.00

single_pulse.ex2

$0.00 \mathrm{MHz}$

$0.00 \mathrm{KHz}$

$0.0 \mathrm{~Hz}$

90 us

E:\K006_[12] paracyclophane-c

$70.30 \mathrm{KHz}$

$33.1 \mathrm{~Hz}$

0

0

$\mathrm{O} \mathrm{Hz}$

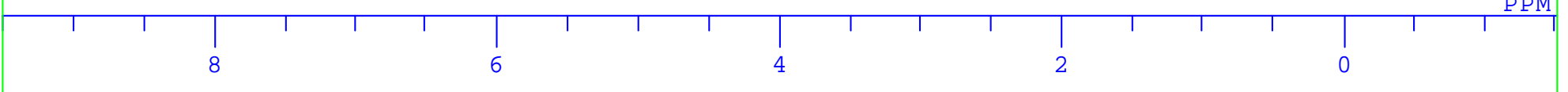


F: \K006_[12\}paracyclophane-diol-13C.1 MIDORI

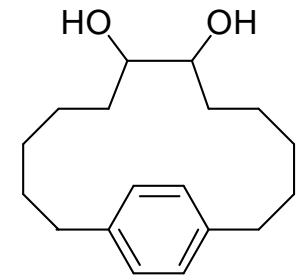

$3 e$
DATIM

MENUF

OBNUC

OFR

OBSET

OBFIN

PW1

DEADT

PREDL

IWT

POINT

SPO

TIMES

DUMMY

FREQU

FLT

DELAY

ACOTM

$\mathrm{PD}$

ADBIT

RGAIN

$B F$

$\mathrm{T} 1$

T2

EXMOD
EXPCM

EXP CM

IFR

IRSET

IRFIN

IRRPW

IRATN

DFILE $S F$

LKSET

LKF IN

LKLEV

LGAIN

LKPHS

LKS I G

CSPED
$3 c$

$125.77 \mathrm{MHz}$

$.87 \mathrm{KHz}$

$4.2 \mathrm{~Hz}$

3.8 us

0.0 us

$0.0000 \mathrm{~ms}$

$1.0 \mathrm{sec}$

26214

26214

$31446.1 \mathrm{~Hz}$ $158000 \mathrm{~Hz}$

20.7 us

$0.834 \mathrm{sec}$

$2.000 \mathrm{sec}$

16.00

2000000

$0.12 \mathrm{~Hz}$

0.00

0.00

$$
90.00
$$$$
100.00
$$

single_pulse_dec

$0.00 \mathrm{MHz}$

$0.00 \mathrm{KHz}$

$0.0 \mathrm{~Hz}$

90 us

F: \K006_[12\}paracyclophane-c

$70.30 \mathrm{KHz}$

$33.1 \mathrm{~Hz}$

0

0

0

$\mathrm{OHz}$

If 
$F: \backslash$ ' uŠ $\cdot \check{S} \hat{1}$ " \pm "ü $\backslash 7$ TBDPSfsfifR $\bullet\left[f<\_\right.$PROTON. 1

K025-381-A-ECA500-CDCL3

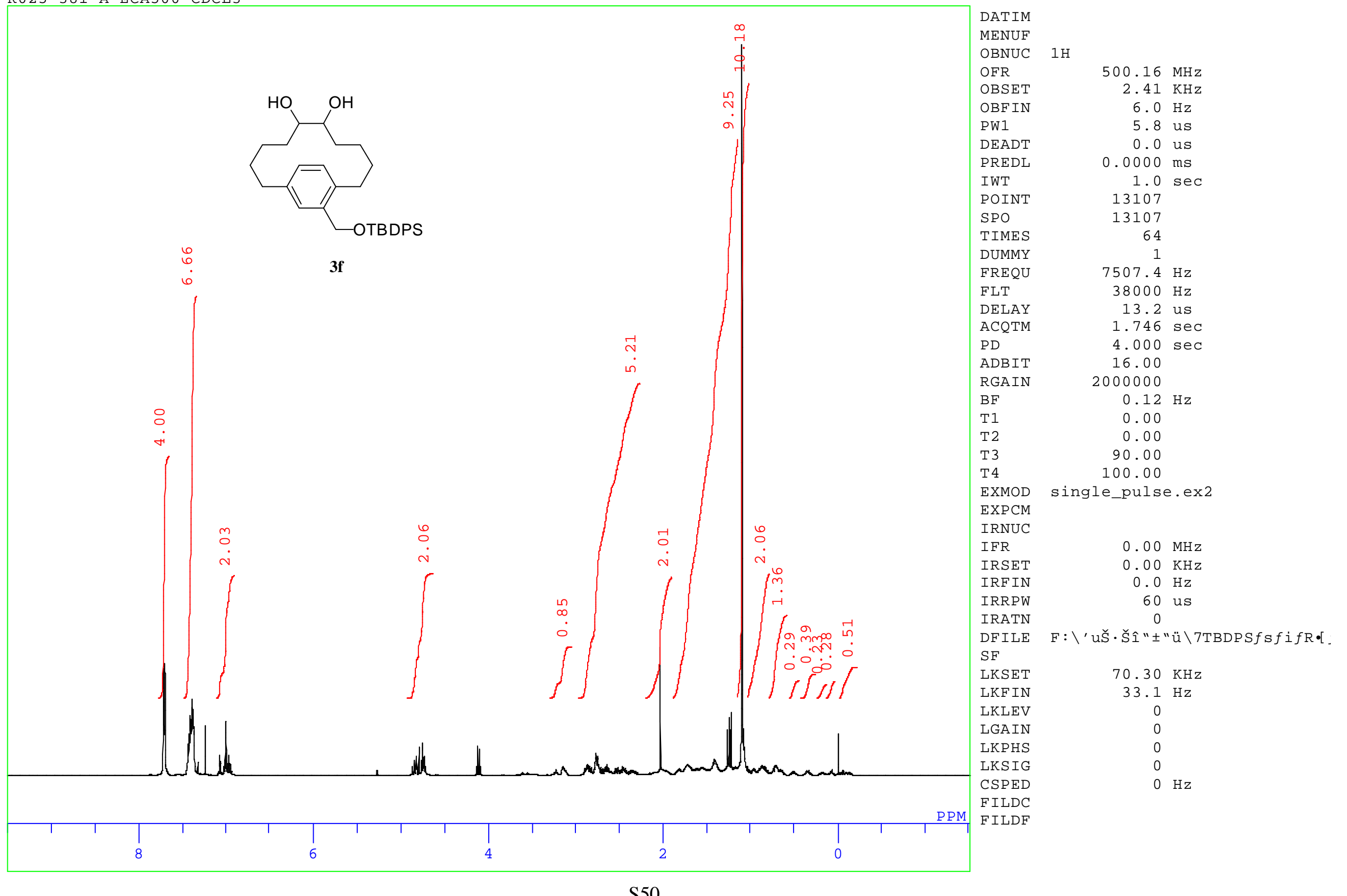




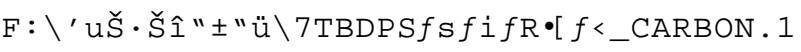

K025-381-A-ECA500-CDCL 3

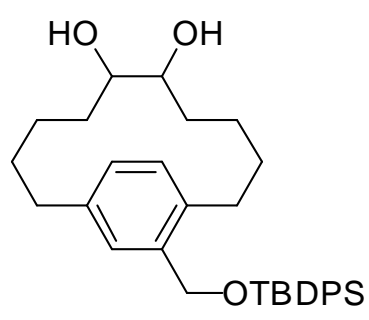

3f
DAT IM

MENUF

OBNUC

OFR

OBSET

OBFIN

PW1

DEADT

PREDI

IWT

POINT

SPO

TIMES

DUMMY

FREQU

FLT

DELAY

ACOTM

$\mathrm{PD}$

ADBIT

RGAIN

$B F$

$\mathrm{T} 1$

T2

$\mathrm{T} 3$

EXMOD

EXPCM

IFR

IRSET

IRF IN

IRRPW

IRATN

DF I LE

SF

LKSET

LKF IN

LKLEV

LGAIN

LKPHS

LKS I G

CSPED

FILDC
$13 c$

$125.77 \mathrm{MHz}$

$7.87 \mathrm{KHz}$

$4.2 \mathrm{~Hz}$

4.1 us

0.0 us

$0.0000 \mathrm{~ms}$

$1.0 \mathrm{sec}$

26214

26214

256

$31446.1 \mathrm{~Hz}$

$158000 \mathrm{~Hz}$

20.7 us

$0.834 \mathrm{sec}$

$4.000 \mathrm{sec}$

16.00

2000000

$0.12 \mathrm{~Hz}$

0.00

0.00

90.00

100.00

single_pulse_dec

$0.00 \mathrm{MHz}$

$0.00 \mathrm{KHz}$

$0.0 \mathrm{~Hz}$

0

: $\backslash$ ' uŠ $• \check{S} \hat{1} " \pm$ "ü $\backslash 7 \mathrm{TBDPSfs}$ fifR $\bullet[$

$70.30 \mathrm{KHz}$

$33.1 \mathrm{~Hz}$

0

0

0

$\mathrm{O} \mathrm{Hz}$

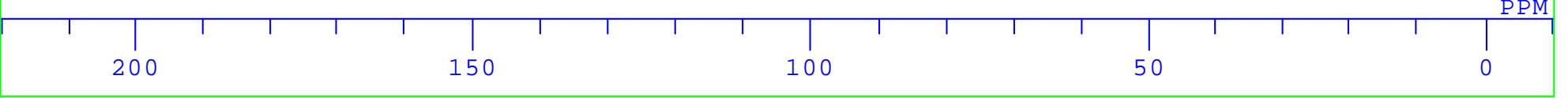


F : \V, $\mu$, cftfH $f<f \_\backslash c y c l o p h a n e-d i o l-B n \_P R O T O N .1$ cyclophane-diol-Bn-ECA500-CDCl3

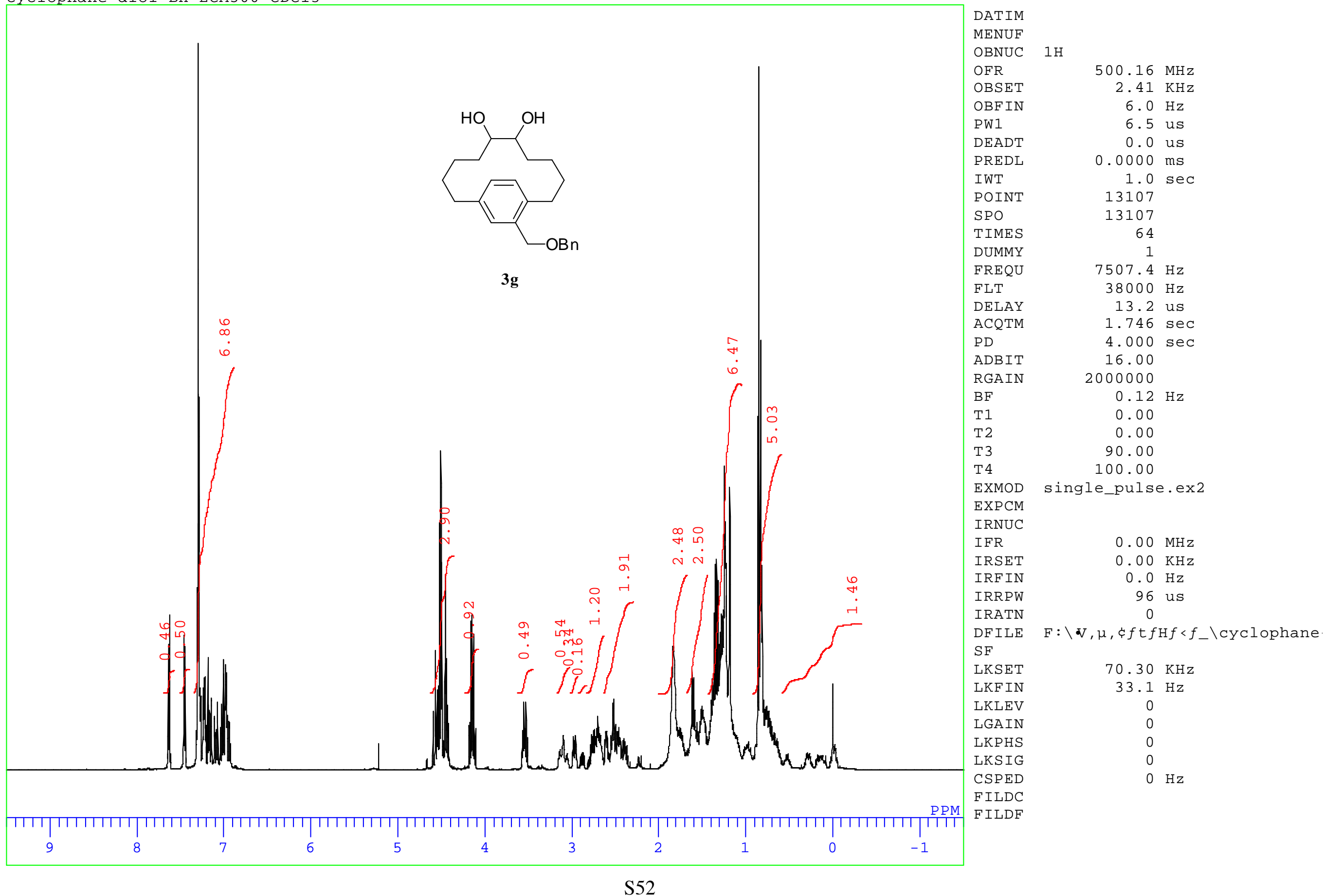




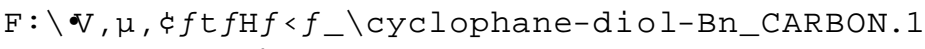
cyclophane-diol-Bn-ECA500-CDCl3

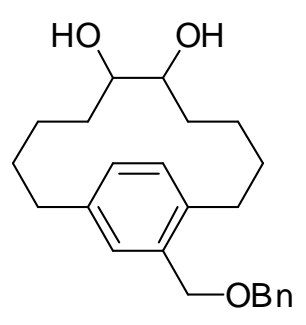

$3 \mathrm{~g}$

4.0 us

0.0 us

$0.00 \mathrm{MHz}$

$0.00 \mathrm{KHz}$

$0.0 \mathrm{~Hz}$

96 us

$F: \backslash \boldsymbol{d}, \mu, \xi f t f H f<f \_\backslash c y c l o p h a n e$

$70.30 \mathrm{KHz}$

$33.1 \mathrm{~Hz}$

0

0

$\mathrm{O} \mathrm{Hz}$ 
F: \K006_[10 (6+4)] paracyclophane-diol-1H.1

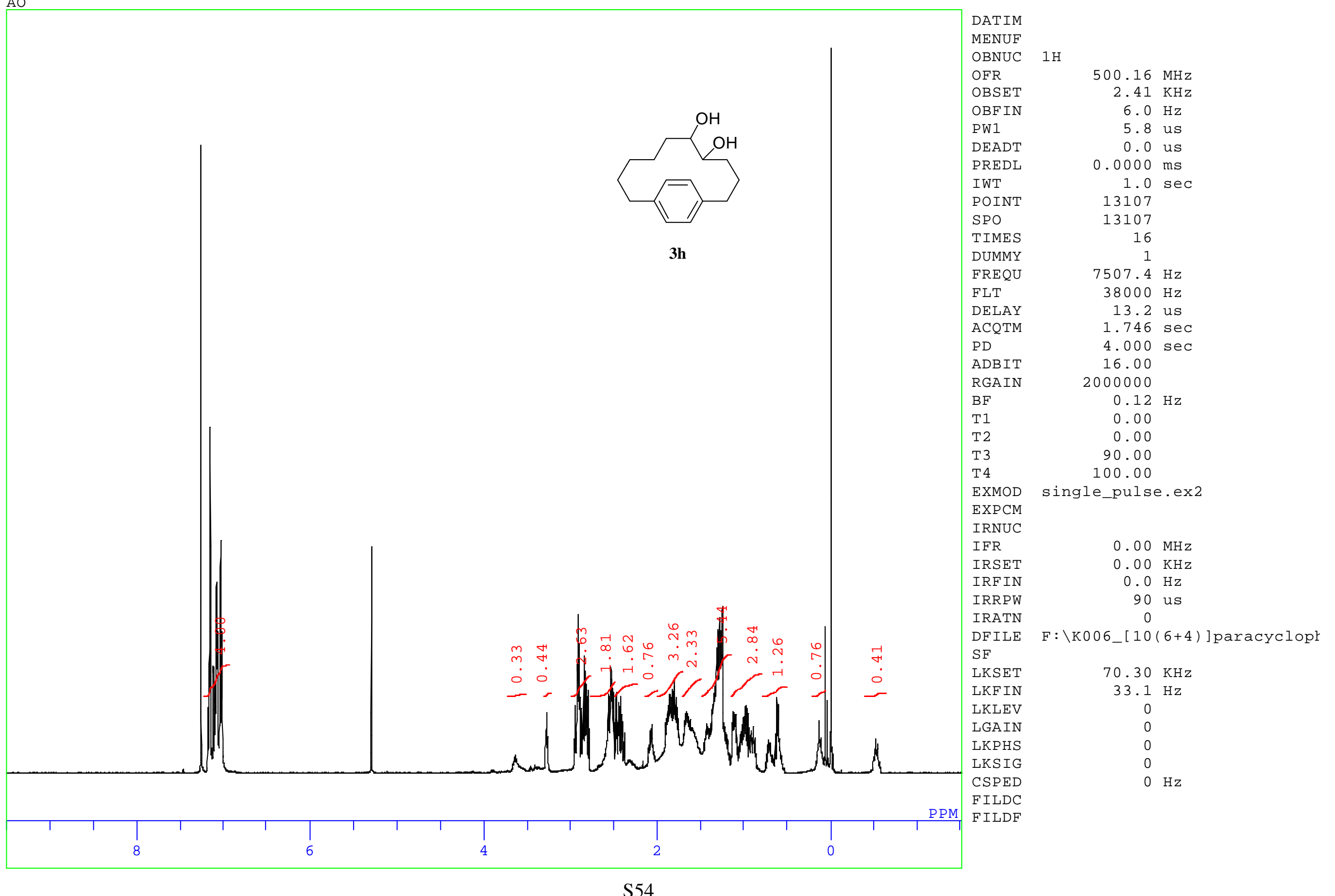


F : \K006_[10(6+4)] paracyclophane-diol-13C.1

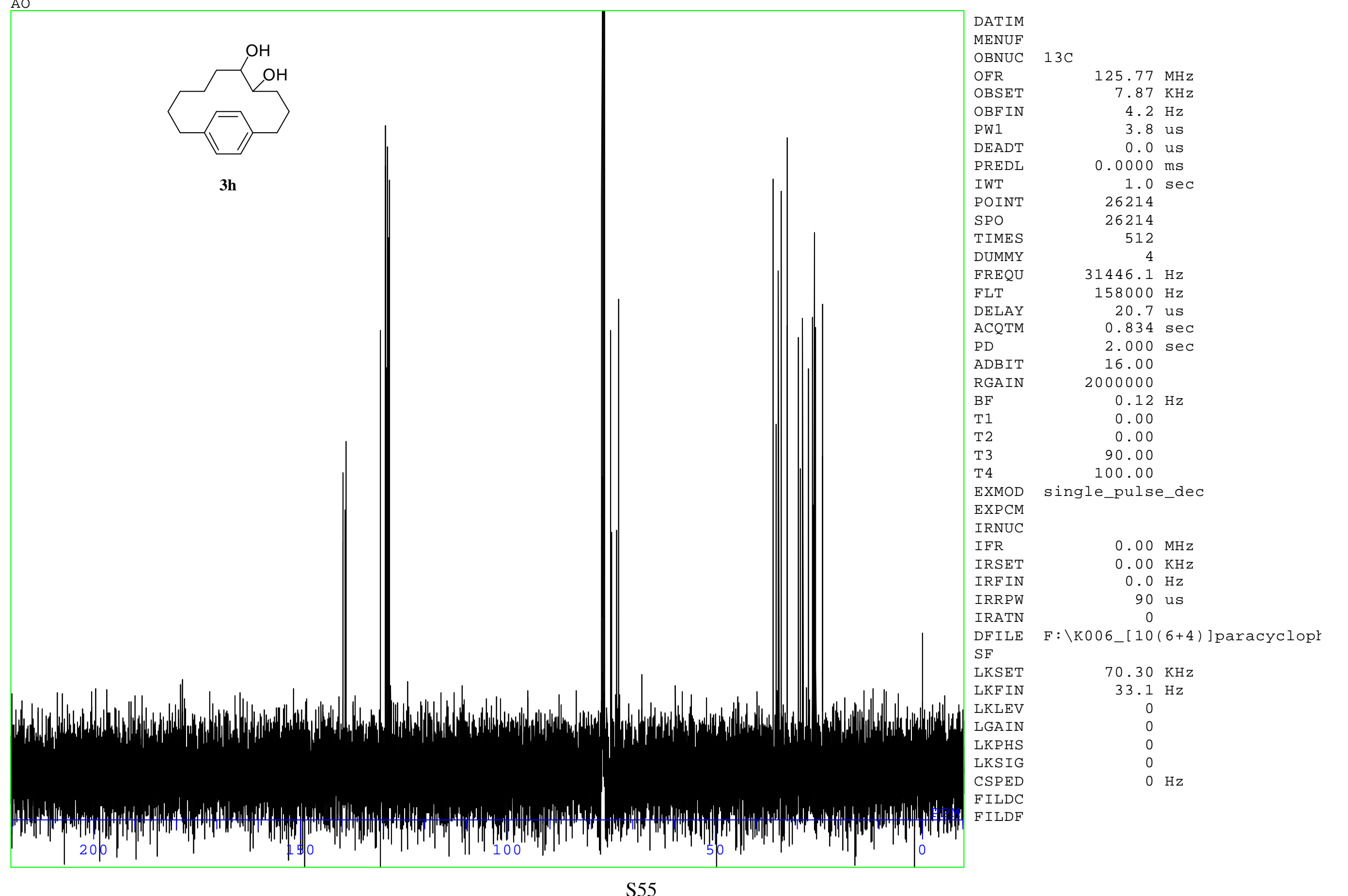


F: \[10,10] paracyclophane-diolefin_PROTON.1 ueda

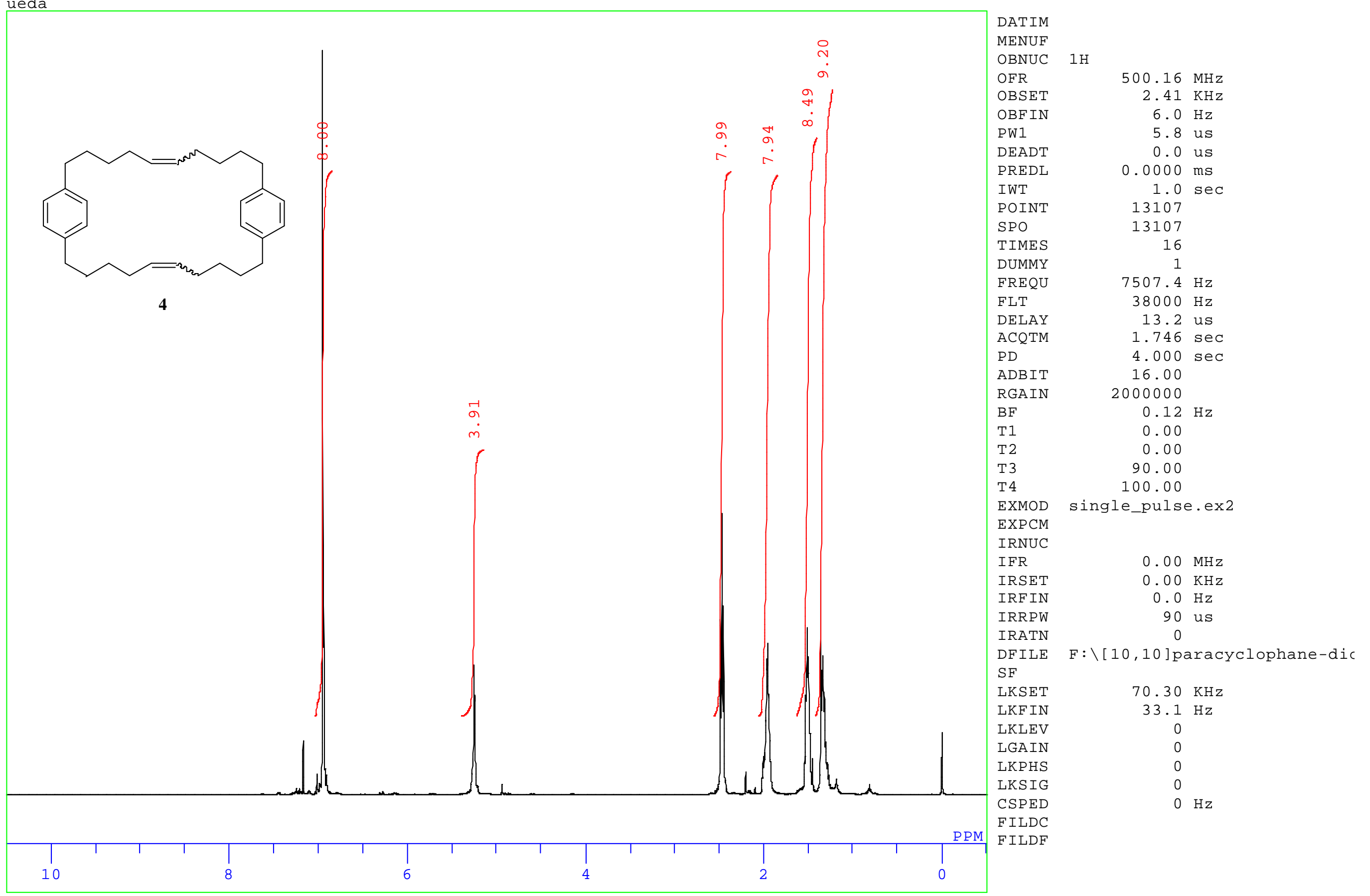


F: \[10,10] paracyclophane-diolefin_CARBON.1 ueda

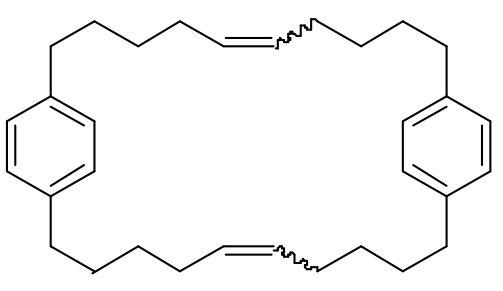

4
DAT IM

MENUF

OBNUC

OFR

OBSET

OBF IN

PW1

DEADT

PREDI

IWT

POINT

SPO

TIMES

DUMMY

FREQU

FLT

DELAY

ACOTM

PD

ADBIT

RGAIN

$B F$

$\mathrm{T} 1$

$\mathrm{T} 2$

EXMOD

EXPCM

IRNUC

IF $R$

IRSET

I RF IN

IRRPW

IRATN

DF I LE

SF

LKSET

LKF IN

LKLEV

LGAIN

LKPHS

LKSIG

CSPED

FILDC

PPM FILDF
$3 c$

$125.77 \mathrm{MHz}$

$.87 \mathrm{KHz}$

$4.2 \mathrm{~Hz}$

3.8 us

0.0 us

$0.0000 \mathrm{~ms}$

$1.0 \mathrm{sec}$

26214

26214

180

$31446.1 \mathrm{~Hz}$

$158000 \mathrm{~Hz}$

20.7 us

$0.834 \mathrm{sec}$

$2.000 \mathrm{sec}$

16.00

2000000

$0.12 \mathrm{~Hz}$

0.00

0.00

90.00

100.00

single_pulse_dec

$0.00 \mathrm{MHz}$

$0.00 \mathrm{KHz}$

$0.0 \mathrm{~Hz}$

90 us

F: $\backslash[10,10]$ paracyclophane-dic

$70.30 \mathrm{KHz}$

$33.1 \mathrm{~Hz}$

0

0

0

$\mathrm{O} \mathrm{Hz}$

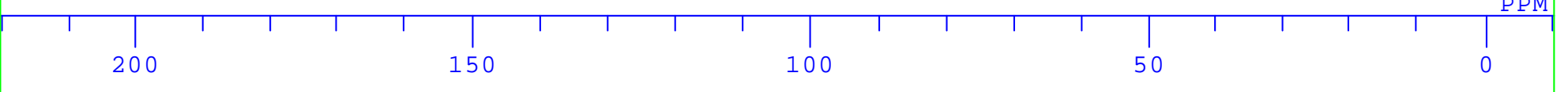


F: \K006-[10] paracyclophane-1H.1 SIRO

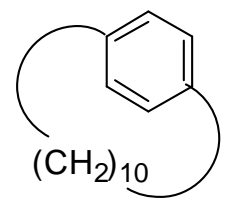

$5 c$
DATIM

MENUF

OBNUC $1 \mathrm{H}$

OFR

OBSET

OBFIN

PW1

DEADT

PREDL

IWT

POINT

SPO

TIMES

DUMMY

FREQU

FLT

DELAY

ACOTM

$\mathrm{PD}$

ADBIT

RGAIN

$B F$

$\mathrm{T} 1$

T2

T4

EXMOD

IRNUC

IFR

IRSET

IRF IN

IRRPW

IRATN

DF I LE

SF

LKSET

LKF IN

LKLEV

LGAIN

LKPHS

LKS I G

CSPED

FILDC

PPM FILDF
$500.16 \mathrm{MHz}$ $2.41 \mathrm{KHz}$

$6.0 \mathrm{~Hz}$

5.8 us

0.0 us

$0.0000 \mathrm{~ms}$

$1.0 \mathrm{sec}$

13107

13107

16

$7507.4 \mathrm{~Hz}$

$38000 \mathrm{~Hz}$

13.2 us

$1.746 \mathrm{sec}$

$4.000 \mathrm{sec}$

16.00

2000000

$0.12 \mathrm{~Hz}$

0.00

0.00

90.00

100.00

single_pulse.ex2

$0.00 \mathrm{MHz}$

$0.00 \mathrm{KHz}$

$0.0 \mathrm{~Hz}$

90 us

0

F: \K006-[10] paracyclophane-]

$70.30 \mathrm{KHz}$

$33.1 \mathrm{~Hz}$

0

0

0

$\mathrm{O} \mathrm{Hz}$

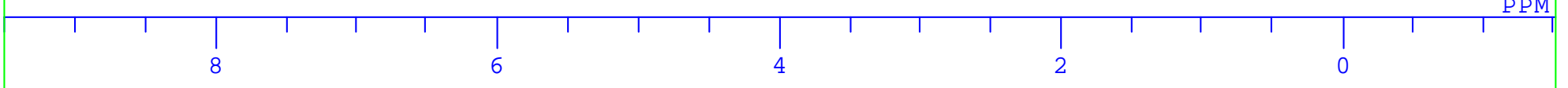


F: \K006-[10] paracyclophane-13C.1 SIRO

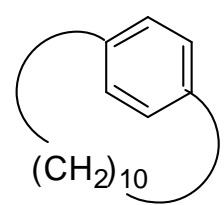

$5 c$
DAT IM

MENUF

OBNUC

OFR

OBSET

OBFIN

PW1

DEADT

PREDL

IWT

POINT

SPO

TIMES

DUMMY

FREQU

FLT

DELAY

ACOTM

$\mathrm{PD}$

ADBIT

RGAIN

$B F$

$\mathrm{T} 1$

$\mathrm{T} 2$

T4

EXMOD
EXPCM

EXPCM

IFR

IRSET

IRFIN

IRRPW

IRATN

DFILE

SF

LKSET

LKF IN

LKLEV

LGAIN

LKPHS

LKS I G

CSPED

FILDC

PPM FILDF
$3 c$

$125.77 \mathrm{MHz}$

$7.87 \mathrm{KHz}$

$4.2 \mathrm{~Hz}$

3.8 us

0.0 us

$0.0000 \mathrm{~ms}$

$1.0 \mathrm{sec}$

26214

26214

16

$31446.1 \mathrm{~Hz}$

$158000 \mathrm{~Hz}$

20.7 us

$0.834 \mathrm{sec}$

$2.000 \mathrm{sec}$

16.00

2000000

$0.12 \mathrm{~Hz}$

0.00

0.00

90.00

100.00

single_pulse_dec

$0.00 \mathrm{MHz}$

$0.00 \mathrm{KHz}$

$0.0 \mathrm{~Hz}$

90 us

F:\K006-[10]paracyclophane-]

$70.30 \mathrm{KHz}$

$33.1 \mathrm{~Hz}$

0

0

0

$\mathrm{OHz}$

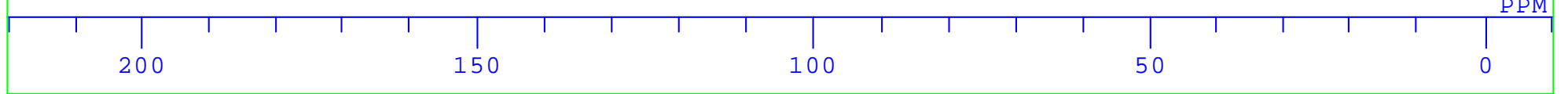


F : \K006-[11]_paracyclophane-1H.1 K00 6

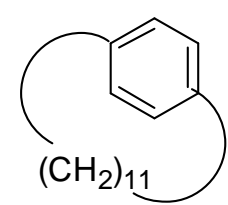

5d
DATIM

MENUF

OBNUC

OFR

OBSET

OBFIN

PW1

DEADT

PREDL

IWT

POINT

SPO

TIMES

DUMMY

FREQU

FLT

DELAY

ACOTM

$\mathrm{PD}$

ADBIT

RGAIN

BF

$\mathrm{T} 1$

T2

T4

EXMOD

IRNUC

IFR

IRSET

IRE IN

IRRPW

IRATN

DFILE

SF

LKSET

LKF IN

LKLEV

LGAIN

LRHS

LKS I G

CSPED

FILDC

FILDF
$500.16 \mathrm{MHz}$ $2.41 \mathrm{KHz}$

$6.0 \mathrm{~Hz}$

5.8 us

0.0 us

$0.0000 \mathrm{~ms}$

$1.0 \mathrm{sec}$

13107

13107

16

$7507.4 \mathrm{~Hz}$ $38000 \mathrm{~Hz}$

13.2 us

$1.746 \mathrm{sec}$

$4.000 \mathrm{sec}$

16.00

2000000

$0.12 \mathrm{~Hz}$

0.00

0.00

90.00

100.00

single_pulse.ex2

$0.00 \mathrm{MHz}$

$0.00 \mathrm{KHz}$

$0.0 \mathrm{~Hz}$

90 us

: $\backslash$ K006-[11]_paracyclophane-

$70.30 \mathrm{KHz}$

$33.1 \mathrm{~Hz}$

0

0

0

$0 \mathrm{~Hz}$

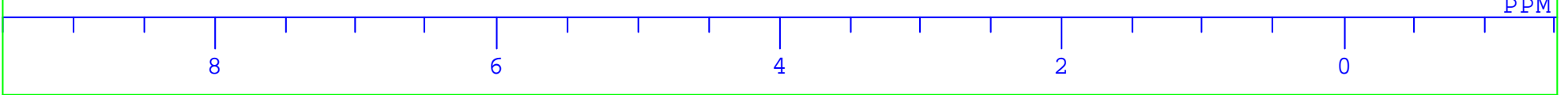


$\mathrm{F}: \backslash \mathrm{K} 006-[11]$ _paracyclophane-13C.1

K006

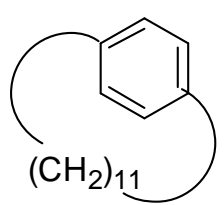

5d
DATIM

MENUF

OBNUC

OFR

OBSET

OBFIN

PW1

DEADT

PREDL

IWT

POINT

SPO

TIMES

DUMMY

FREQU

FLT

DELAY

ACQTM

$\mathrm{PD}$

ADBIT

RGAIN

$B F$

$\mathrm{T} 1$

T2

T3

EXMOD

EXPCM

IRNUC

IF $R$

IRSET

IRFIN

IRRPW

IRATN

DFILE

SF

LKSET

LKF IN

LKLEV

LGAIN

LKP HS

LKSIG

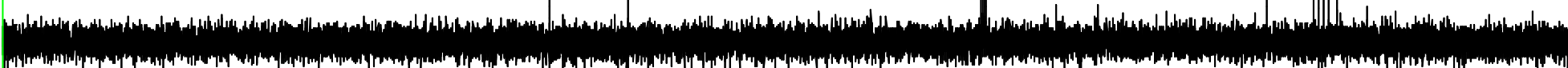

CSPED

FILDC

PPM FILDF
$3 c$

$125.77 \mathrm{MHz}$

$.87 \mathrm{KHz}$

$4.2 \mathrm{~Hz}$

3.8 us

0.0 us

$0.0000 \mathrm{~ms}$

$1.0 \mathrm{sec}$

26214

26214

$31446.1 \mathrm{~Hz}$

$158000 \mathrm{~Hz}$

20.7 us

$0.834 \mathrm{sec}$

$2.000 \mathrm{sec}$

16.00

2000000

$0.12 \mathrm{~Hz}$

0.00

0.00

90.00

100.00

single_pulse_dec

$0.00 \mathrm{MHz}$

$0.00 \mathrm{KHz}$

$0.0 \mathrm{~Hz}$

90 us

F: \K006-[11]_paracyclophane-

$70.30 \mathrm{KHz}$

$33.1 \mathrm{~Hz}$

0

0

0

$\mathrm{O} \mathrm{Hz}$

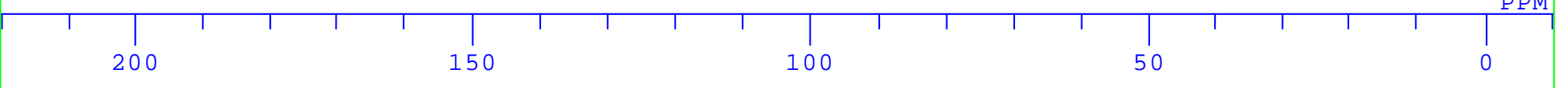




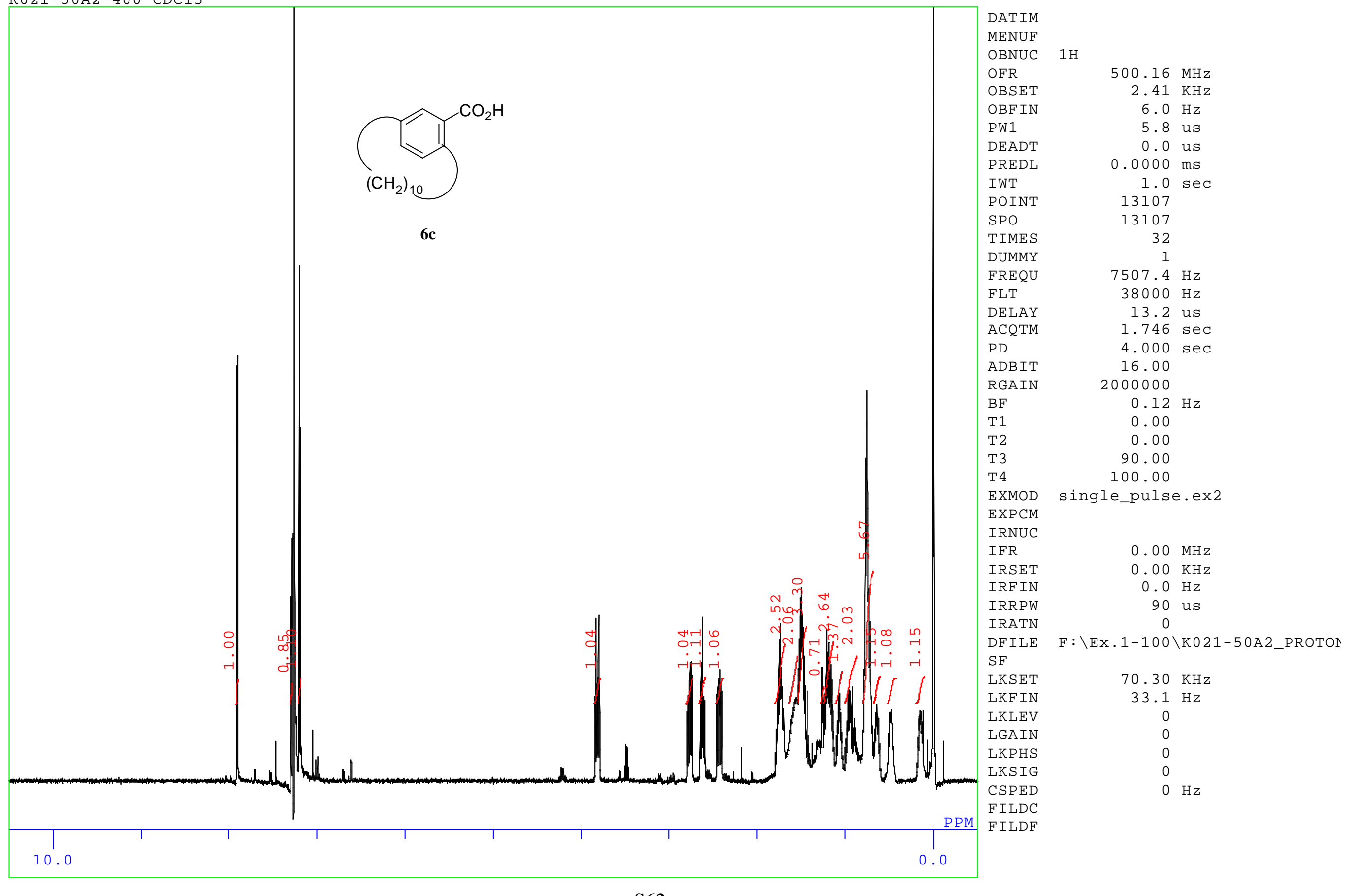




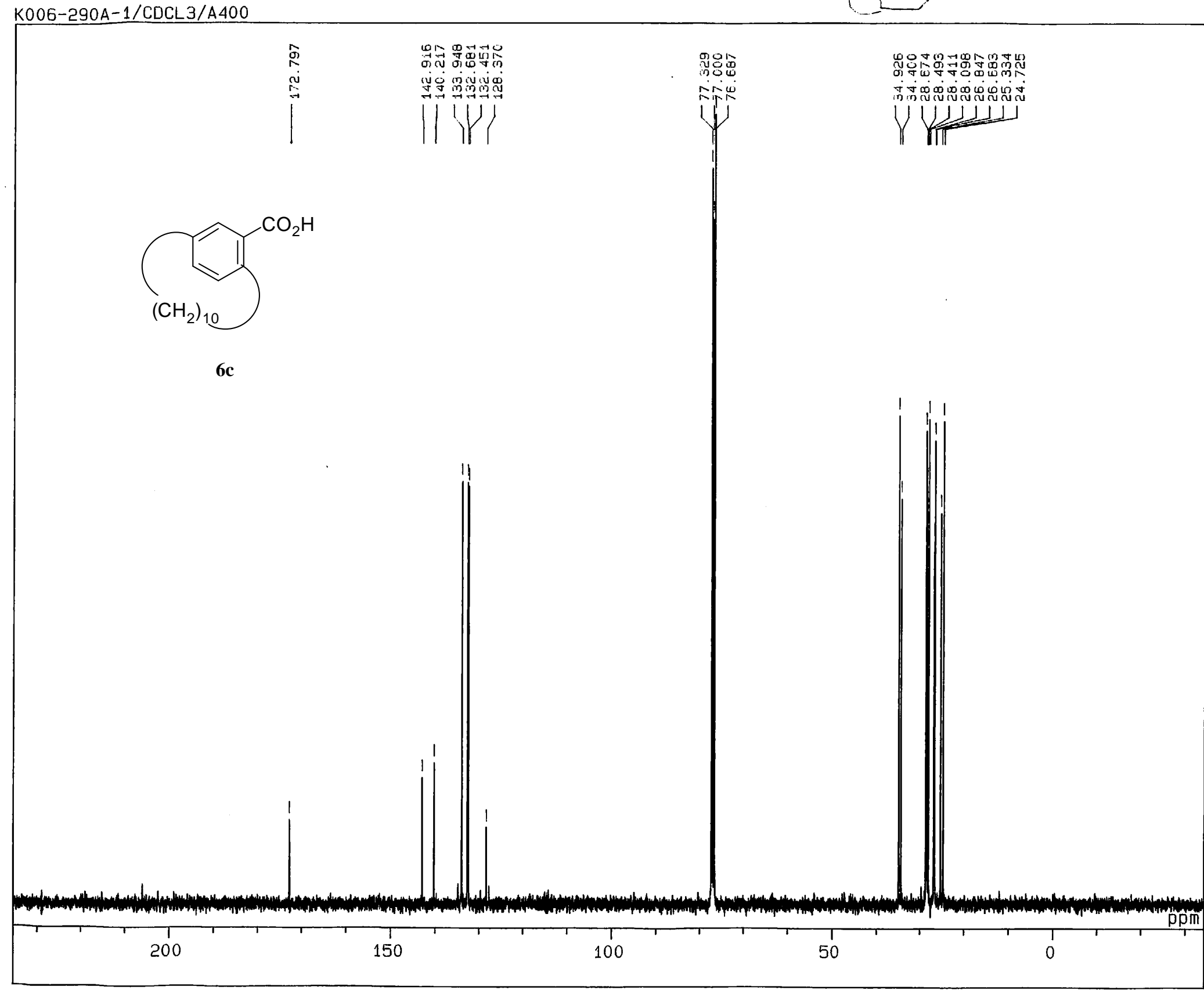

16-DEC-2003 09: 29: 36.92 DFILE : ALPHA SF ILE

COMNT : K006-290A-1/CDCL3/A400 EXMOD : SING

IRMOD : BCM

POINT : 16384

FREQU : $27100.27 \mathrm{~Hz}$

SCANS : $\quad 4096$

DUMMY : $\quad 4$

ACQTM : $\quad 0.6046 \mathrm{sec}$

PD : $\quad 1.7909 \mathrm{sec}$

RGAIN : $\quad 23$

PW1 : 11.30 usec

OBNUC : $13 \mathrm{C}$

OBFAQ : $100.40 \mathrm{MHz}$

OBSET : $135500.00 \mathrm{~Hz}$

IRNUC : $1 \mathrm{H}$

$399.65 \mathrm{MHz}$

IRSET : $134300.00 \mathrm{~Hz}$

IRATN: 511

IRRPW : 50.0 usec

IRBP 1: 24

$\begin{array}{ll}\text { IRBP2 } & 6 \\ \text { IRRNS }: & 0\end{array}$

ADBIT : $\quad 16$

CSPED : $\quad 11 \mathrm{~Hz}$

SLVNT : CDCL3

RESOL : $\quad 1.65 \mathrm{~Hz}$

BF : $1.50 \mathrm{~Hz}$

T1 : $\quad 0.00 \%$

T2: $: 0.00 \%$

T4 : $100.00 \%$

REFVL : $177.00 \%$

XEFVL : $77.00 \mathrm{ppm}$

$\begin{array}{rrr}X E & : 27100.27 ~ H z \\ X S & : & 0.00 ~ H z\end{array}$

operator 
$F: \backslash 11 \% \ddot{E}<' \bullet \bullet \backslash 11 \% \ddot{E}<{ }^{\circ}$ Á $\bullet f f \bullet\left[f^{\wedge} \backslash 9 f J f<f\{f\right.$ ' K025-358-A-ECA $500-$ CDCL 3

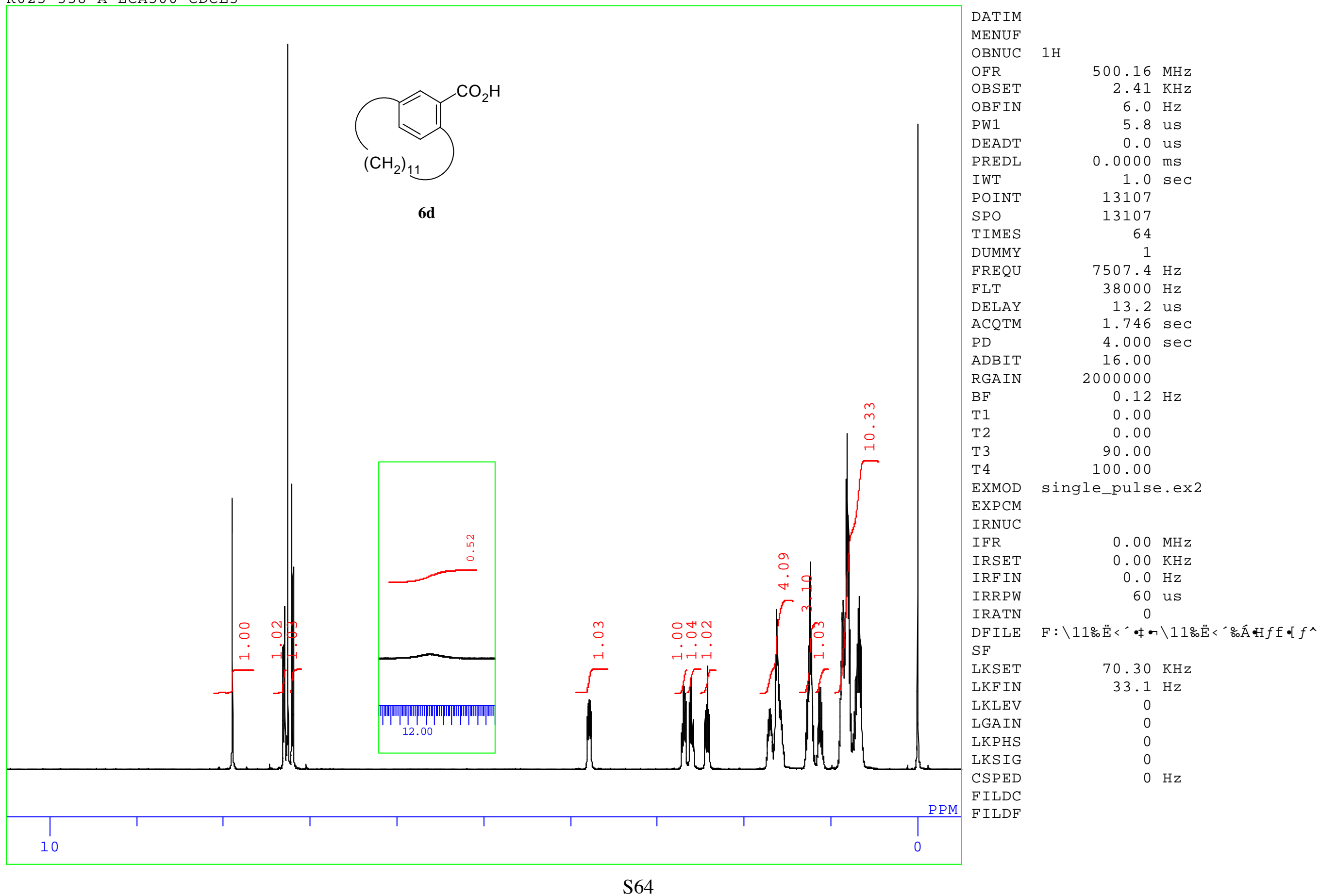




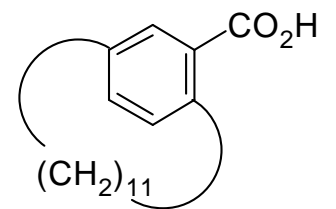

6d
DATIM

MENUF

OBNUC

OFR

OBSET

OBFIN

PW1

DEADT

PREDL

IWT

POINT

SPO

TIMES

DUMMY

FREQU

FLT

DELAY

ACQTM

$\mathrm{PD}$

ADBIT

RGAIN

$B F$

$\mathrm{T} 1$

T2

$\mathrm{T} 3$

$\mathrm{T} 4$

EXMOD

EXPCM

IRNUC

IFR

IRSET

IRFIN

IRRPW

IRATN

DF ILE $\mathrm{SF}$

LKSET

IKETN

LKLEV

LGAIN

LKPHS

LKS I G

CSPED

FILDC

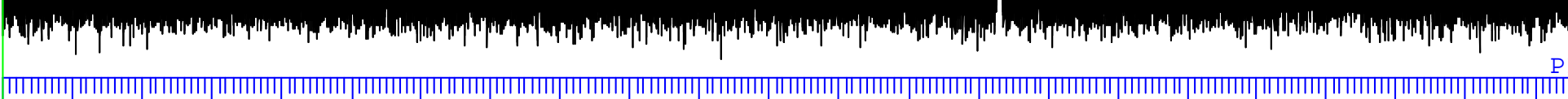

.
$13 c$

$125.77 \mathrm{MHz}$

$.87 \mathrm{KHz}$

$4.2 \mathrm{~Hz}$

4.1 us

0.0 us

$0.0000 \mathrm{~ms}$

$1.0 \mathrm{sec}$

26214

26214

1024
4

$31446.1 \mathrm{~Hz}$

$158000 \mathrm{~Hz}$

20.7 us

$0.834 \mathrm{sec}$

$4.000 \mathrm{sec}$

16.00

2000000

$0.12 \mathrm{~Hz}$

0.00

0.00

90.00

100.00

single_pulse_dec

$0.00 \mathrm{MHz}$

$0.00 \mathrm{KHz}$

$0.0 \mathrm{~Hz}$

$60 \mathrm{u}$

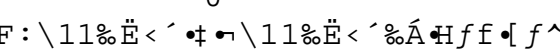

$70.30 \mathrm{KHz}$

$33.1 \mathrm{~Hz}$

0

0

$0 \mathrm{~Hz}$ 
$\mathrm{F}: \backslash \check{Z} \grave{A} \mathscr{E} \pm f \mathrm{~m} \bullet[$ fgNo.1\141`160\K035-154-A_PROTON.als K035-154-A/ECA 500/CDC13

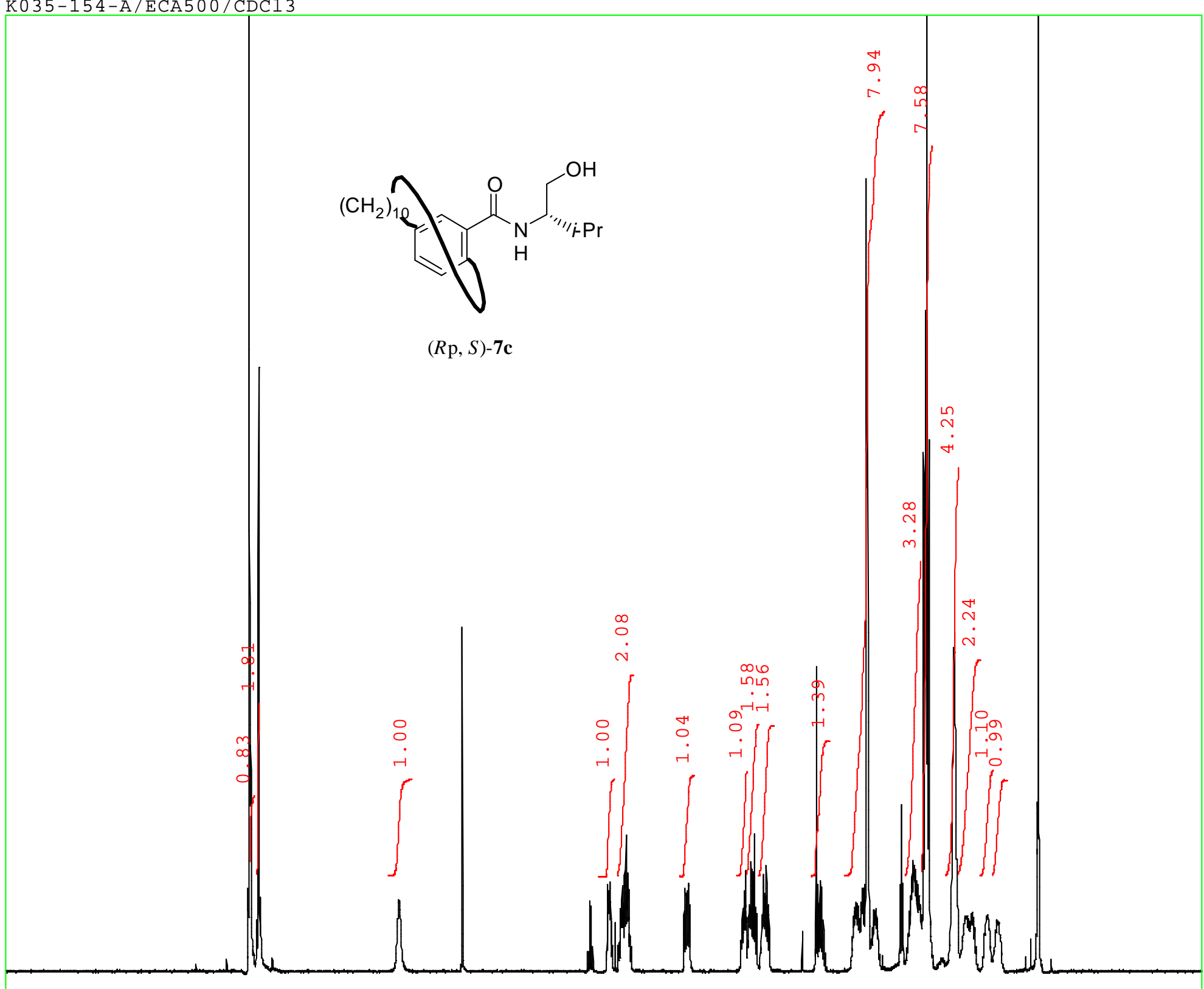

OBF IN

POINT

TIMES

DUMMY

FREQU

KSET

LKF IN

LKLEV

LGA IN

KPHS

LKS I G 


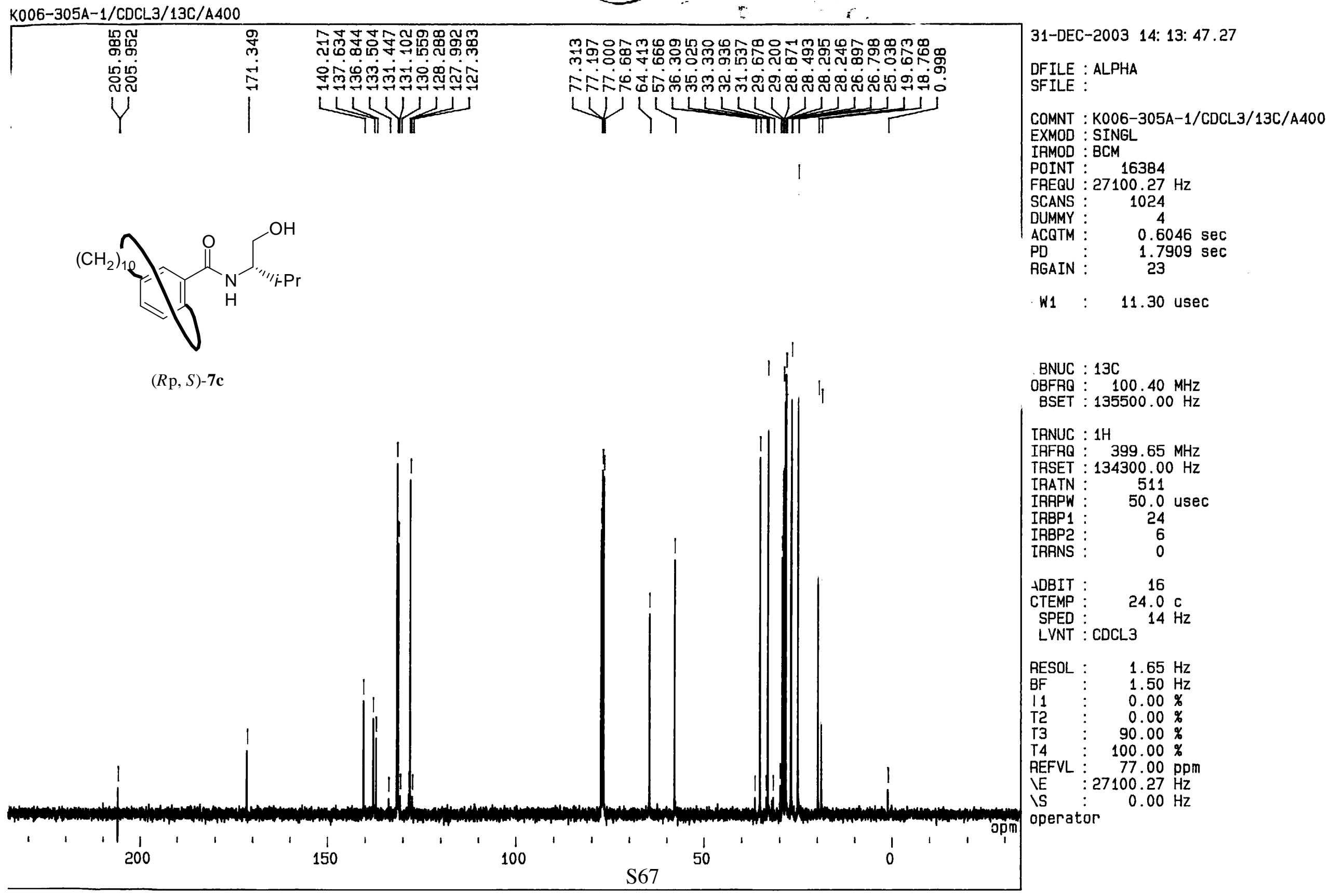


F : $\backslash$ Ex.1-100\K021-52A2_PROTON.als

K021-52A1-500-CDCl3

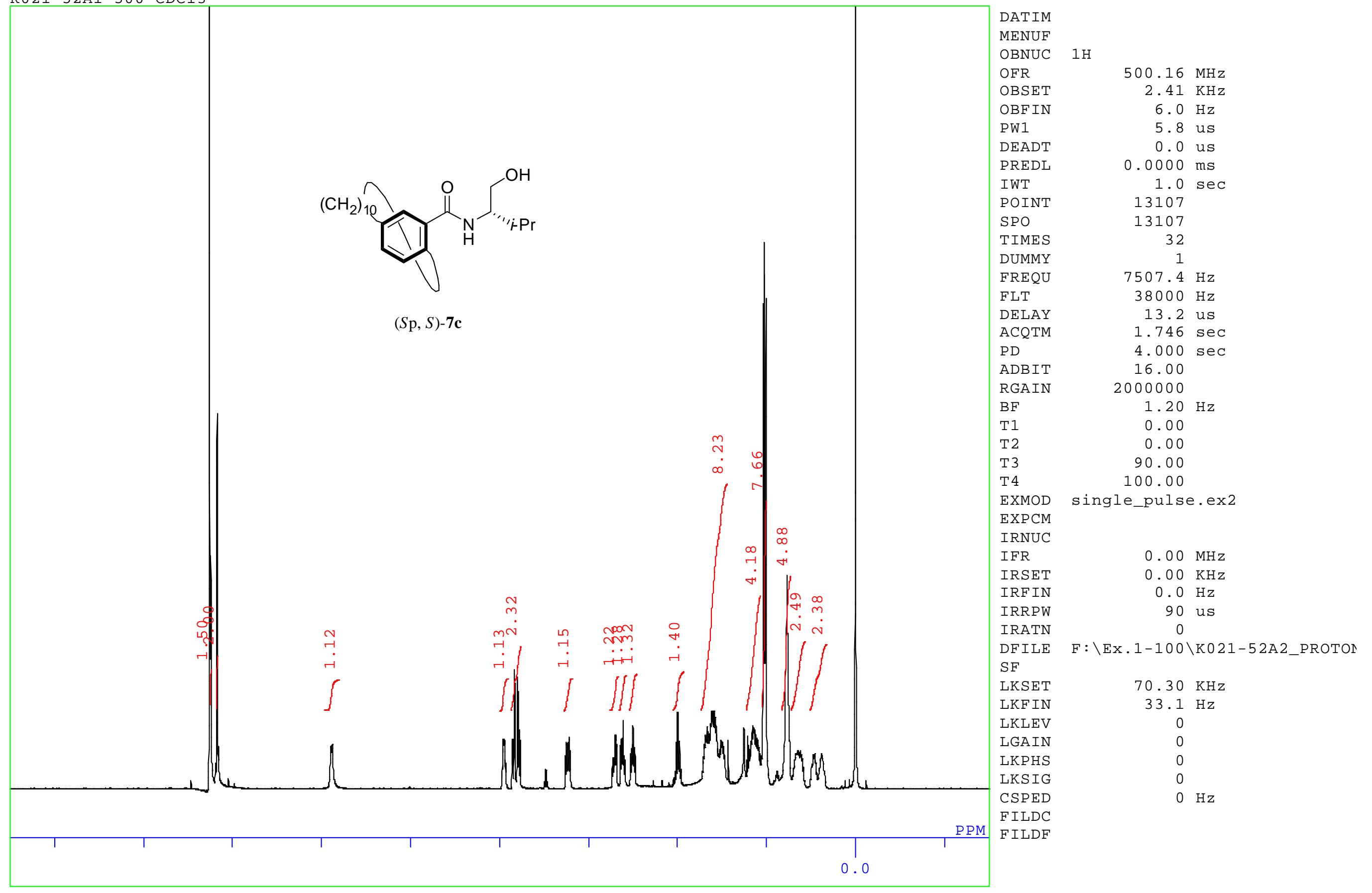



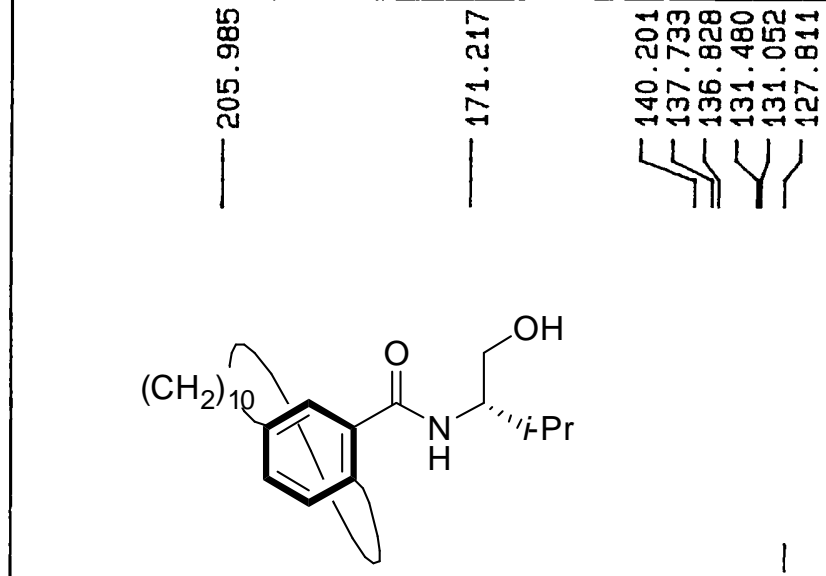

$(S \mathrm{p}, S)-\mathbf{7 c}$

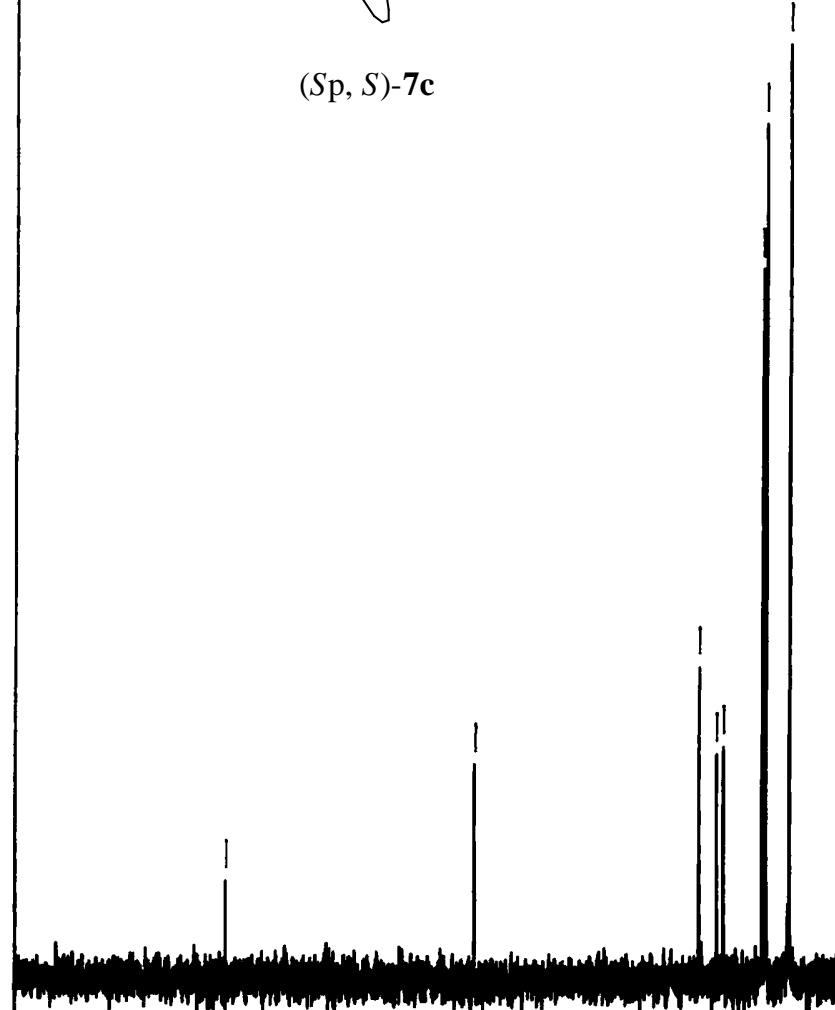

)

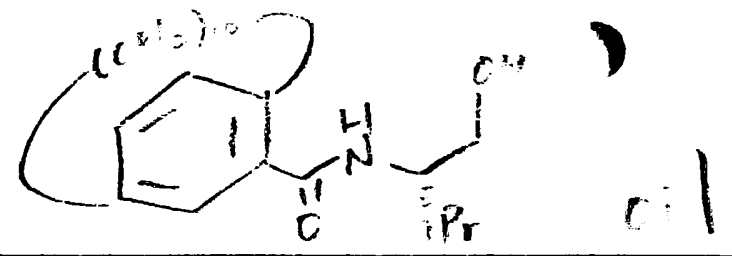

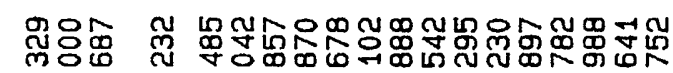

수 ธำ

4114140111

III 11

\|\|

\|\|

$\|$
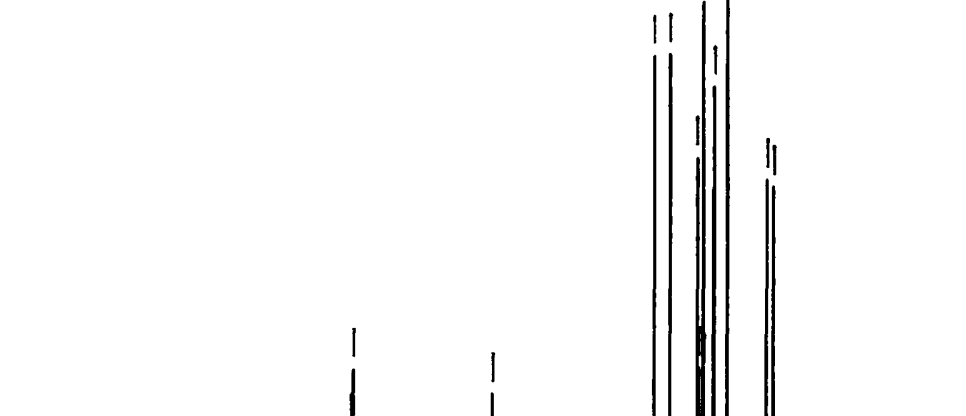

$\begin{array}{r}1 \\ \hline+200\end{array}$

150

100

S69

50

0 


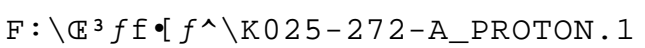

K025-272-A-ECA $400-C D C L 3$

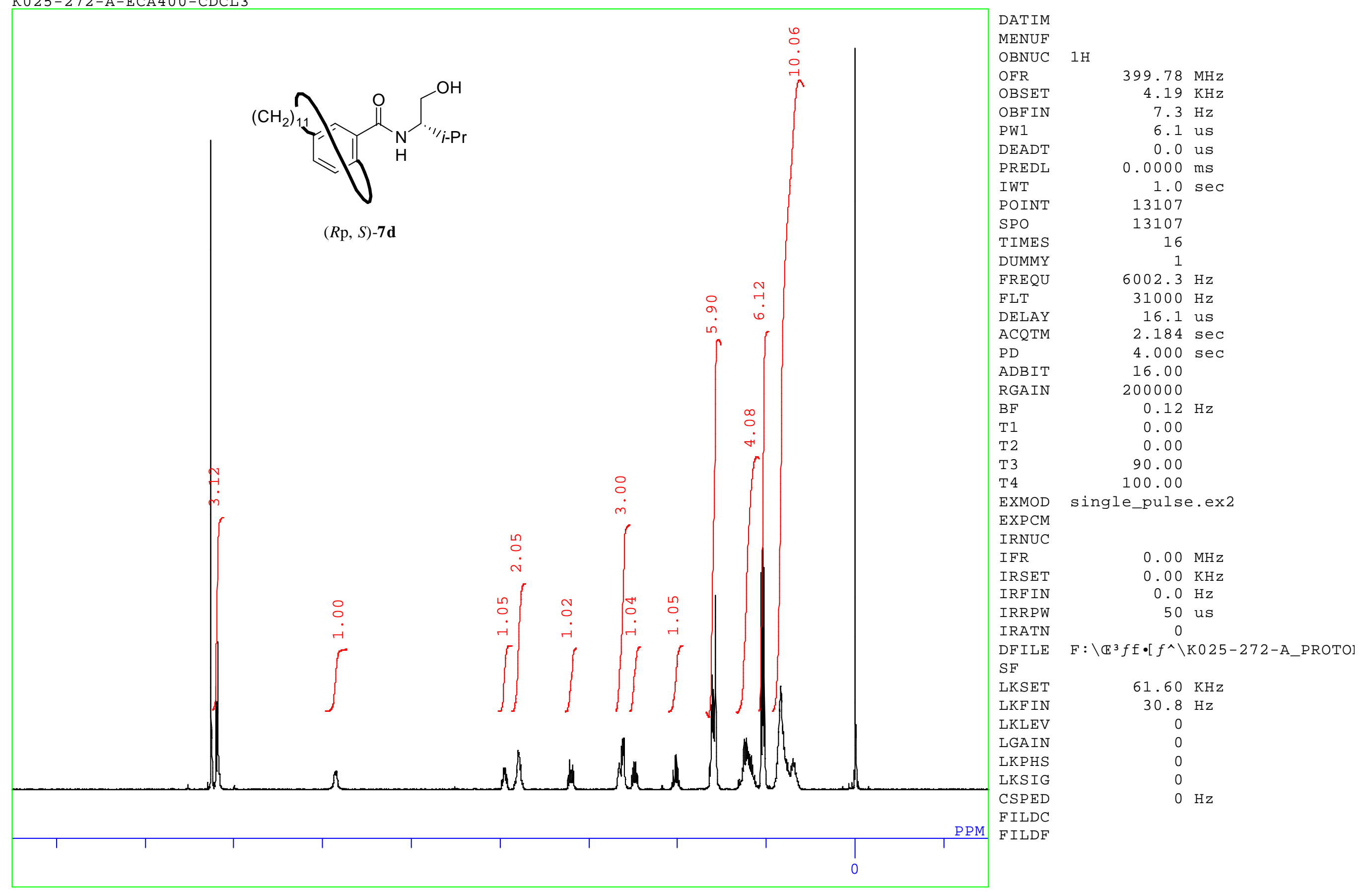




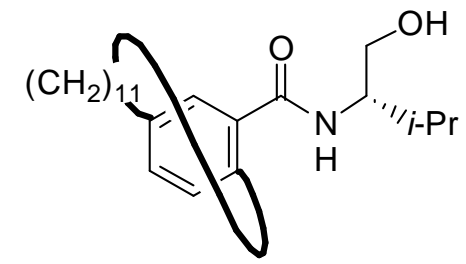

$(R \mathrm{p}, S)-\mathbf{7 d}$

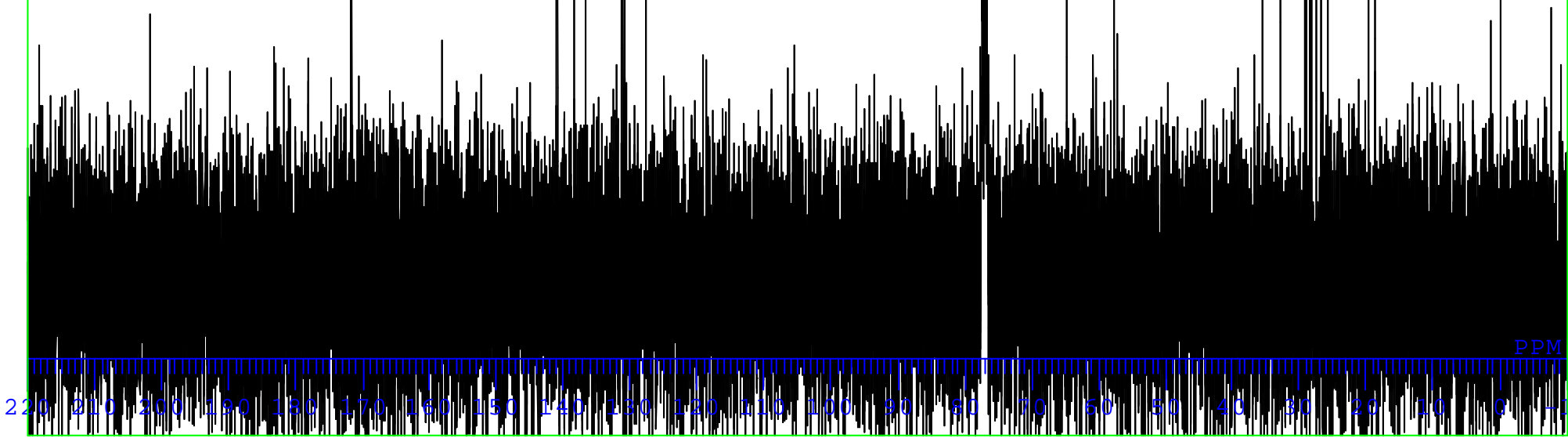

OBNUC

OFR

OBSET

OBFIN

PW1

DEADT

PREDI

POINT

SPO

T IME

DUMMY

FREQU

FLT

DELAY

ACOTM

ADBIT

RGAIN

$B F$

$\mathrm{T} 1$

T2

EXMOD

EXPCM

IRNUC

IFR

IRSET

IRE IN

IRATN

DFILE $S F$

LKFIN

LKLEV

LGAIN

LKPHS

LKS I G

CSPED

FILDC

F I LDF
$13 c$

$100.53 \mathrm{MHz}$

$5.35 \mathrm{KHz}$

$5.9 \mathrm{~Hz}$

3.2 us

0.0 us

$0.0000 \mathrm{~ms}$

$1.0 \mathrm{sec}$

26214

26214

1024

4

$25125.2 \mathrm{~Hz}$

$126000 \mathrm{~Hz}$

21.9 us

$1.043 \mathrm{sec}$

$4.000 \mathrm{sec}$

16.00

150000

$0.12 \mathrm{~Hz}$

0.00

0.00

90.00

100.00

single_pulse_dec

$0.00 \mathrm{MHz}$

$0.00 \mathrm{KHz}$

$0.0 \mathrm{~Hz}$

$\mathrm{F}: \backslash \mathbb{E}^{3} f \mathrm{f} \bullet\left[f^{\wedge} \backslash \mathrm{K} 025-272-\mathrm{A} \_C A R B O\right]$

\section{$61.60 \mathrm{KHz}$}

$30.8 \mathrm{~Hz}$

0

0

$\mathrm{O} \mathrm{Hz}$ 


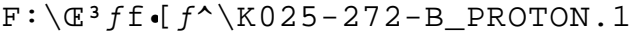

K025-272-B-ECA $400-C D C L 3$

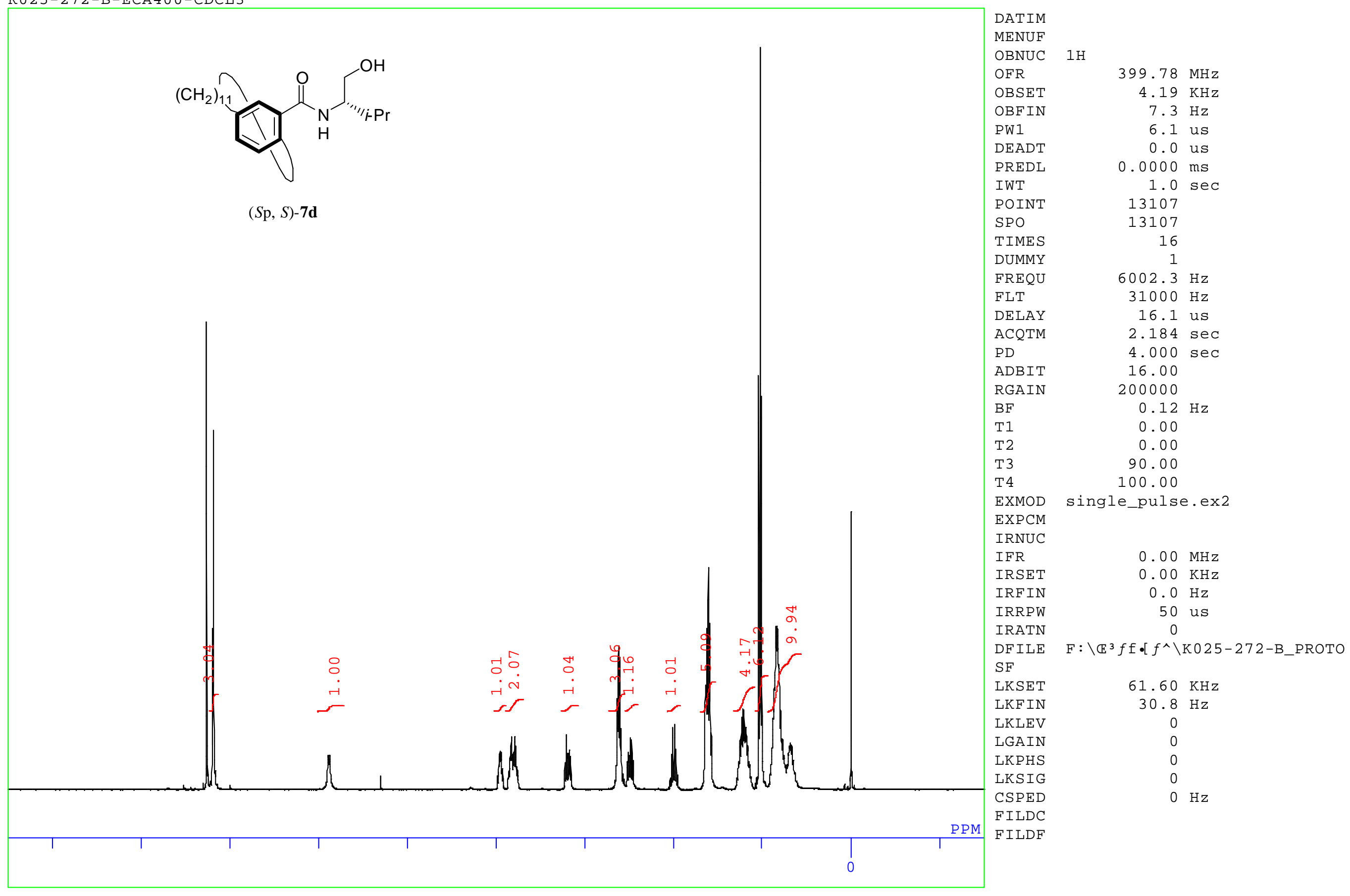


$F: \backslash \mathbb{E}^{3} f f \cdot\left[f^{\wedge} \backslash K 025-272-B \_C A R B O N .1\right.$ K025-272-B-ECA $400-$ CDCL 3

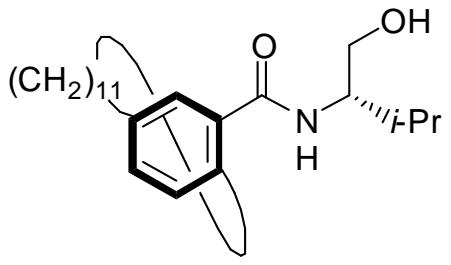

$(S \mathrm{p}, S)-\mathbf{7 d}$

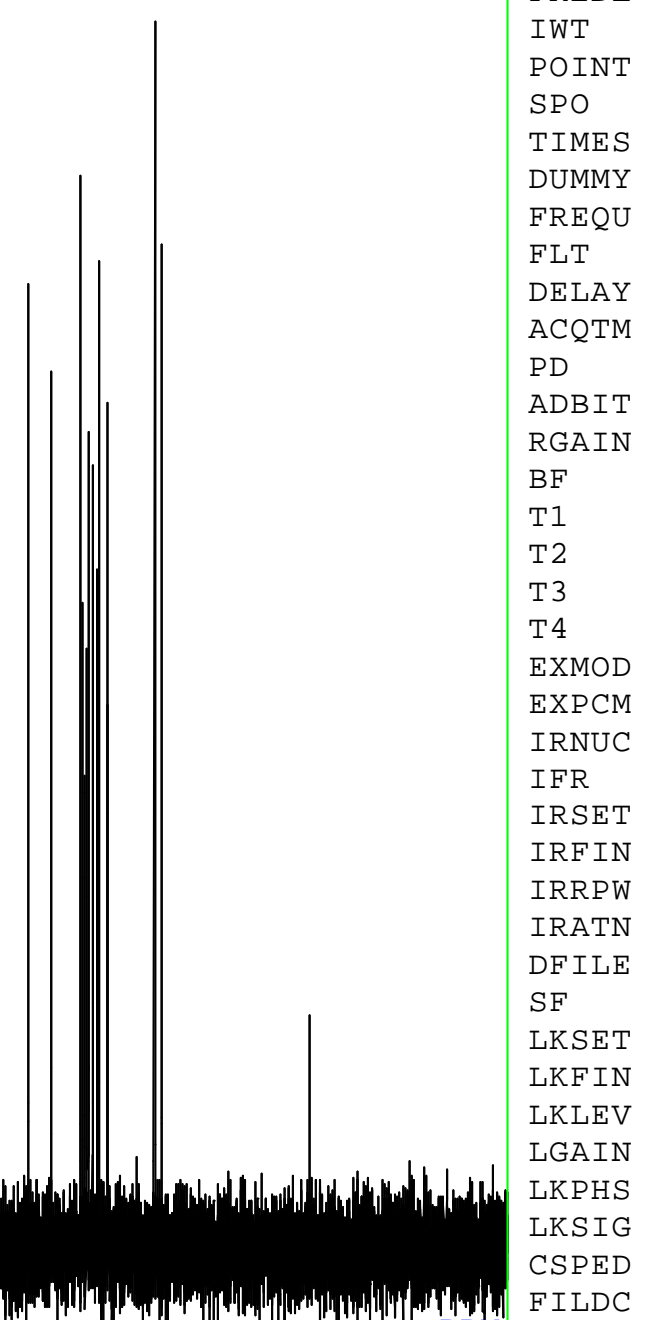

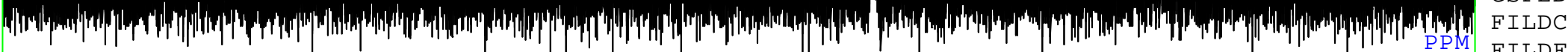

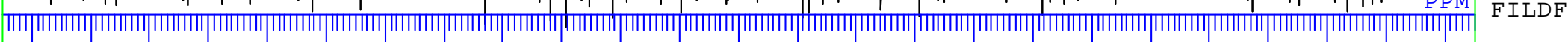
$220210200190180170160150140130120110100 \quad 90$ 
$\mathrm{F}: \backslash \boldsymbol{V}, \mu, \xi f \mathrm{t} f \mathrm{f} f<f_{-} \backslash \mathrm{K} 025-11 \mathrm{R}, \mathrm{S}-\mathrm{tBu}$ _PROTON. 1

K025-11R, S-tBu-ECA 400-CDCL3

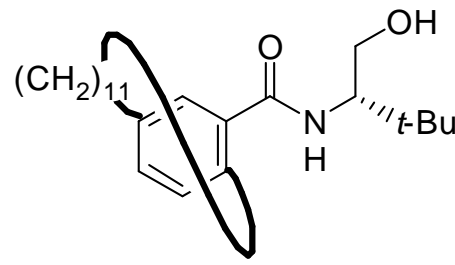

$(R \mathrm{p}, S)-\mathbf{8 d}$

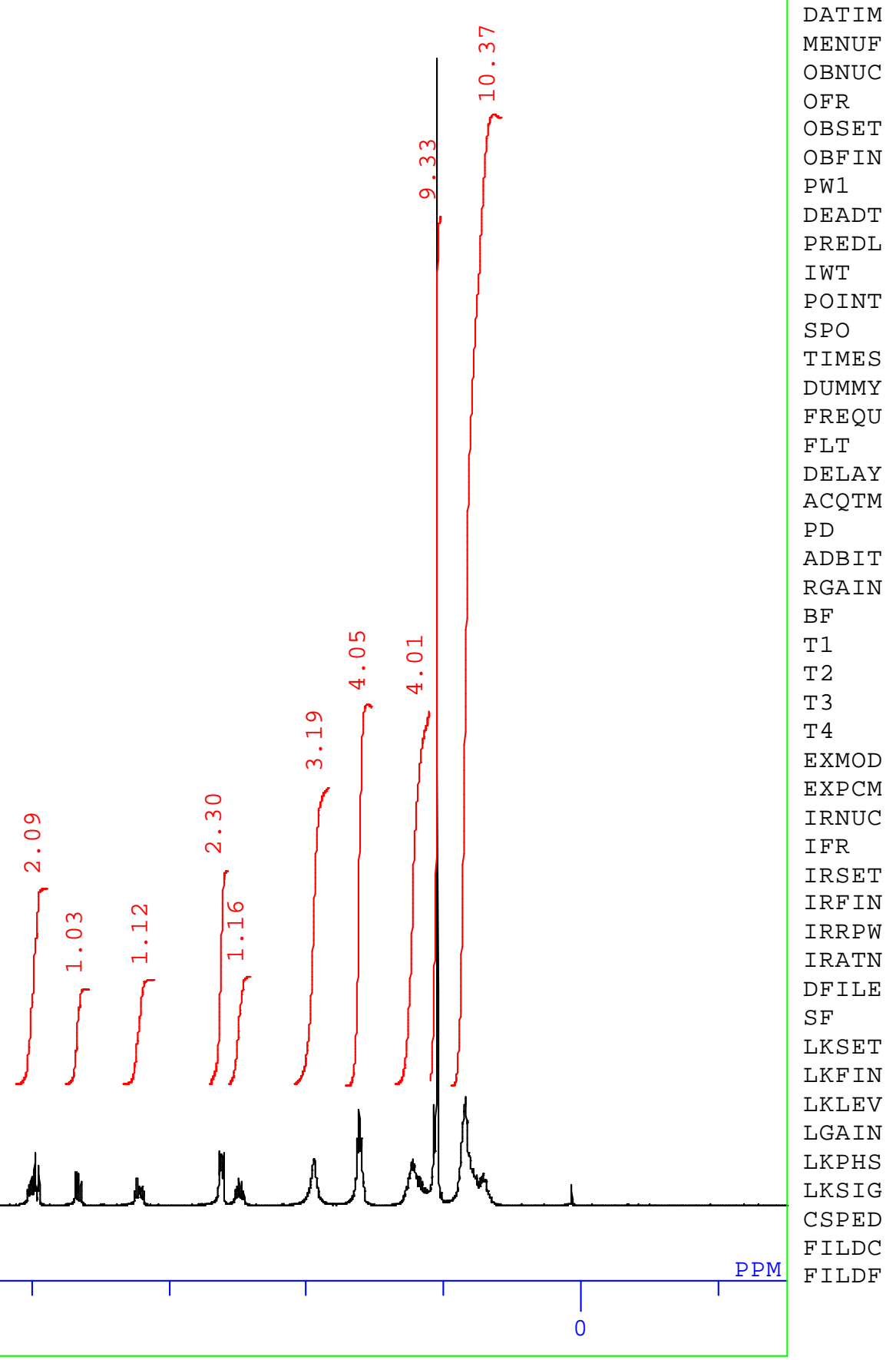

$1 \mathrm{H}$

$399.78 \mathrm{MHz}$ $4.19 \mathrm{KHz}$

$7.3 \mathrm{~Hz}$

5.8 us

0.0 us

$0.0000 \mathrm{~ms}$

$1.0 \mathrm{sec}$

13107

13107

16

$6002.3 \mathrm{~Hz}$

$31000 \mathrm{~Hz}$

16.1 us

$2.184 \mathrm{sec}$

$4.000 \mathrm{sec}$

16.00

200000

$0.12 \mathrm{~Hz}$

0.00

0.00

90.00

100.00

single_pulse.ex2

XXCM

RF IN

KSET

KF IN

GAIN

KSIG

ILDC

$$
\begin{array}{cl}
0.00 & \mathrm{MHz} \\
0.00 & \mathrm{KHz} \\
0.0 & \mathrm{~Hz} \\
50 & \mathrm{us}
\end{array}
$$

$F: \backslash \mathbb{Q}, \mu, \xi f t f H f<f \_\backslash K 025-11 R, S$

\section{$61.60 \mathrm{KHz}$}

$30.8 \mathrm{~Hz}$

0

0

0

$0 \mathrm{~Hz}$ 
F : \k006_358a_1_CARBON.1

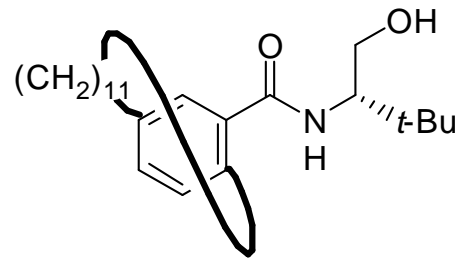

$(R \mathrm{p}, S)-\mathbf{8 d}$
DAT IM

MENUF

OBNUC

OFR

OBSET

OBFIN

PW1

DEADT

PREDL

IWT

POINT

SPO

TIMES

DUMMY

FREQU

FLT

DELAY

ACOTM

PD

ADBIT

RGAIN

$B F$

$\mathrm{T} 1$

T2

T4

EXMOD

IRNUC

IF $R$

IRSET

IRF IN

IRRPW

IRATN

DFILE $S F$

LKSET

LKF IN

LKLEV

LGAIN

LKPHS

LKSIG H TाTाTा|ा

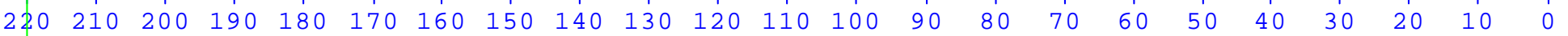

$13 c$

$125.77 \mathrm{MHz}$

$.87 \mathrm{KHz}$

$4.2 \mathrm{~Hz}$

3.8 us

0.0 us

$0.0000 \mathrm{~ms}$

1.0 sec

26214

26214

45

$31446.1 \mathrm{~Hz}$

$158000 \mathrm{~Hz}$

20.7 us

$0.834 \mathrm{sec}$

$2.000 \mathrm{sec}$

16.00

2000000

$0.12 \mathrm{~Hz}$

0.00

0.00

90.00

100.00

single_pulse_dec

$0.00 \mathrm{MHz}$

$0.00 \mathrm{KHz}$

$0.0 \mathrm{~Hz}$

90 us

F : \k006_358a_1_CARBON.1

$70.30 \mathrm{KHz}$

$33.1 \mathrm{~Hz}$

0

0

$0 \mathrm{~Hz}$ 
$\mathrm{F}: \backslash \boldsymbol{V}, \mu, \xi f \mathrm{t} f \mathrm{f} f<f_{-} \backslash \mathrm{K} 025-11 \mathrm{~S}, \mathrm{~S}-\mathrm{tBu}$ _PROTON. 1

K025-11S, S-tBu-ECA 400-CDCL3

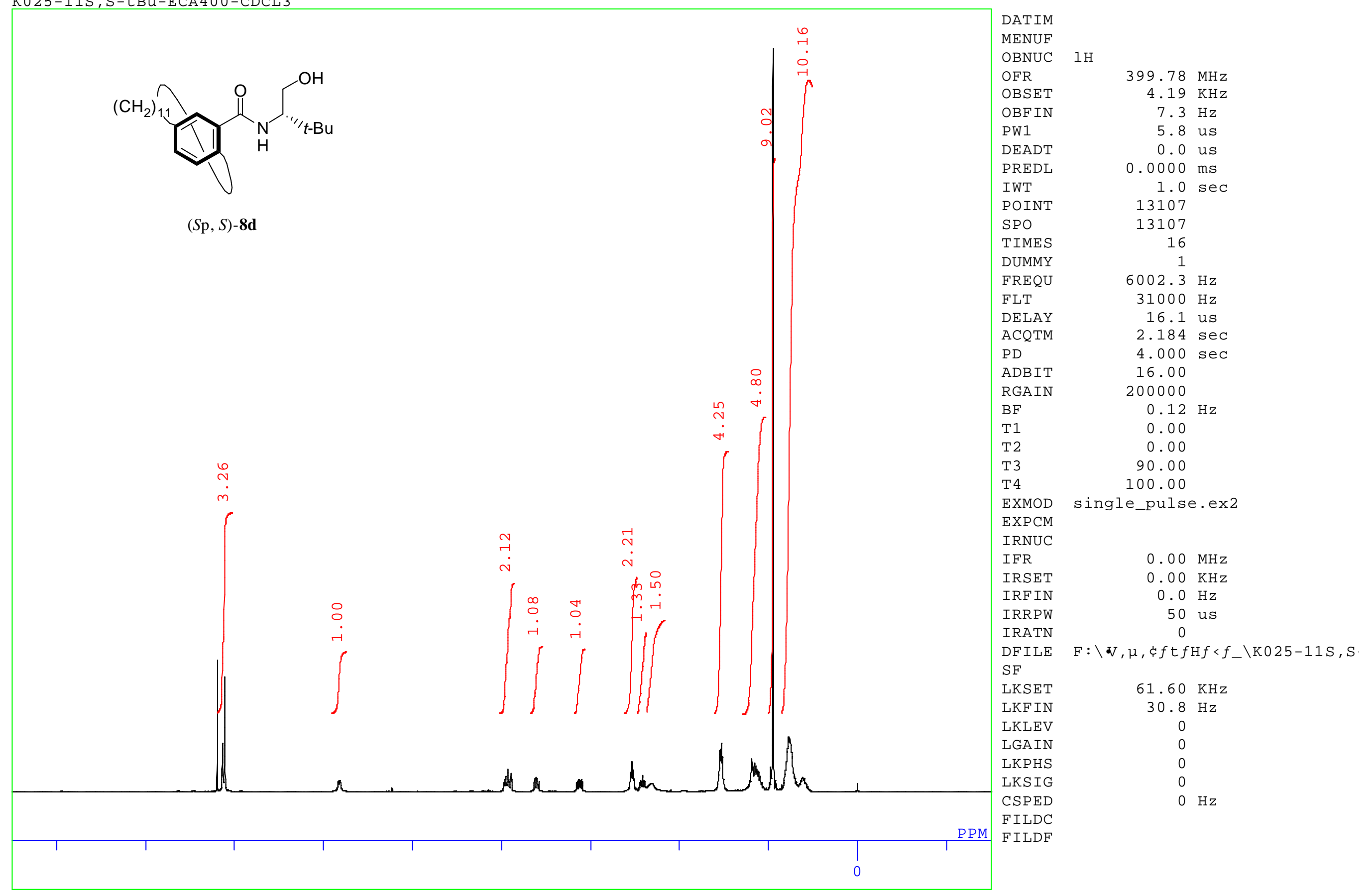


$\mathrm{F}: \backslash \mathcal{V}, \mu, \dot{f t} f \mathrm{H} f<f \_\backslash \mathrm{K} 025-11 \mathrm{~S}, \mathrm{~S}-\mathrm{tBu} \_\mathrm{CARBON} .1$

K025-11S, S-tBu-ECA 400-CDCL3

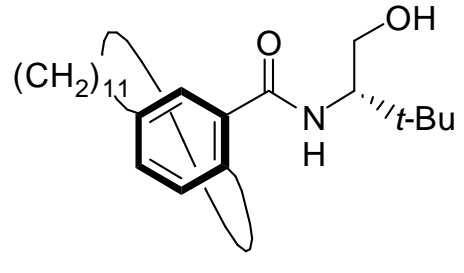

$(S \mathrm{p}, S)-\mathbf{8 d}$
DATIM

MENUF

OBNUC

OFR

OBSET

OBFIN

PW1

DEADT

PREDI

POINT

SPO

TIMES

DUMMY

FREQU

FLT

DELAY

ACOTM

$\mathrm{PD}$

ADBIT

RGAIN

$B F$

$\mathrm{T} 1$

T2

T3

T 4

EXMOD

EXPCM

IFR

IRSET

IRF IN

IRRPW

IRATN

DF I LE

SF

IKSET

LKF IN

LKLEV

LGA IN

LKPHS

LKS I G

CSPED

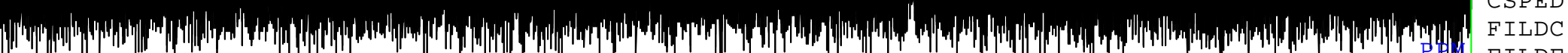
W|

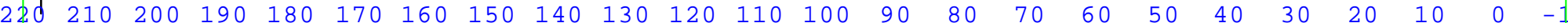

$13 \mathrm{C}$

$100.53 \mathrm{MHz}$ $5.35 \mathrm{KHz}$

$5.9 \mathrm{~Hz}$

3.1 us

0.0 us

$0.0000 \mathrm{~ms}$

$1.0 \mathrm{sec}$

26214

26214

35

$25125.2 \mathrm{~Hz}$

$126000 \mathrm{~Hz}$

21.9 us

$1.043 \mathrm{sec}$

$4.000 \mathrm{sec}$

16.00

150000

$0.12 \mathrm{~Hz}$

0.00

0.00

90.00

100.00

single_pulse_dec

$0.00 \mathrm{MHz}$

$0.00 \mathrm{KHz}$

$0.0 \mathrm{~Hz}$

$F: \backslash \mathbb{d}, \mu, \dot{\phi} f t f H f<f \_\backslash K 025-11 S, S$

\section{$61.60 \mathrm{KHz}$}

$30.8 \mathrm{~Hz}$

0

0

0

$\mathrm{OHz}$ 


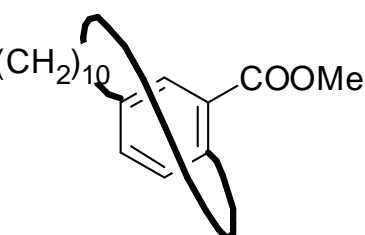

$(R)-(+)-9 \mathbf{c}$

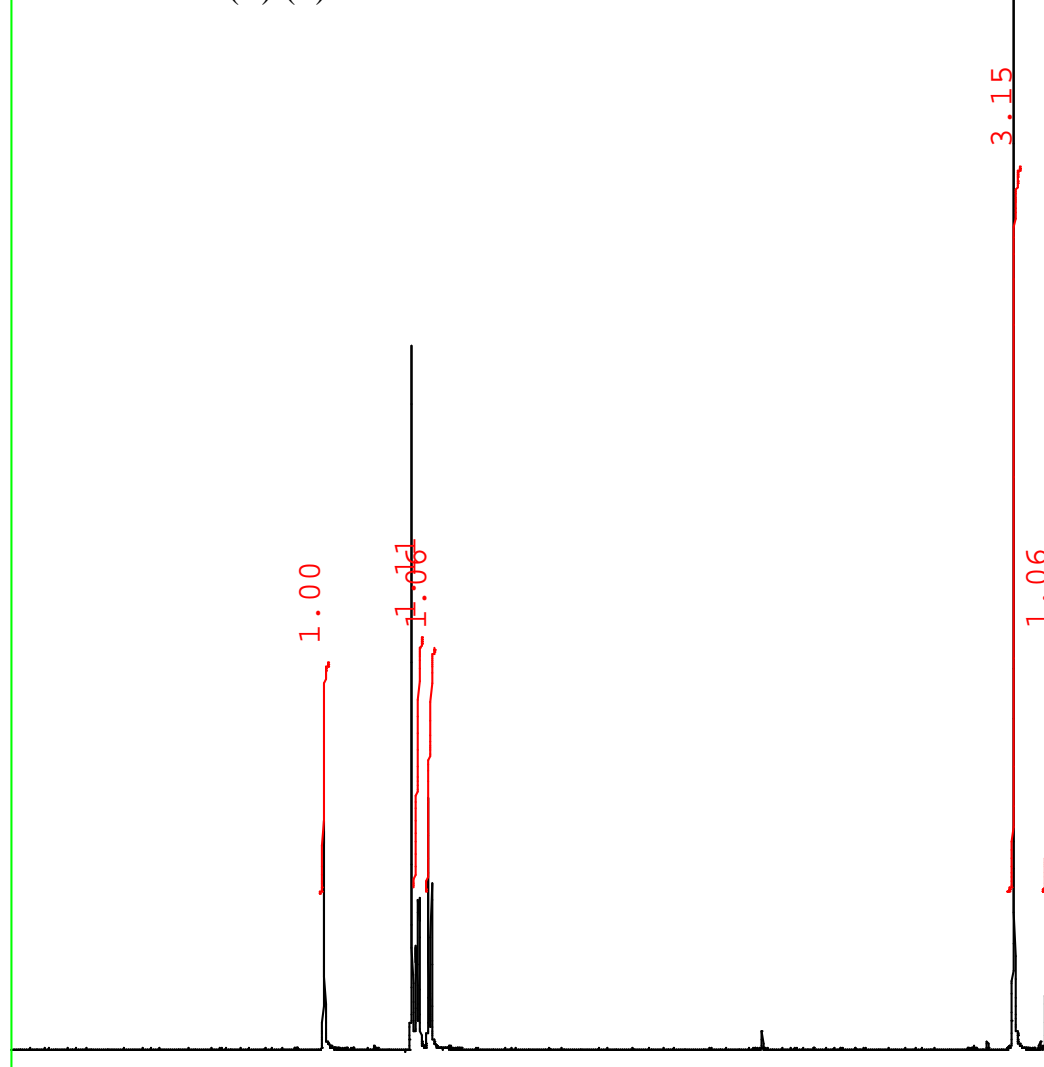

Tा।

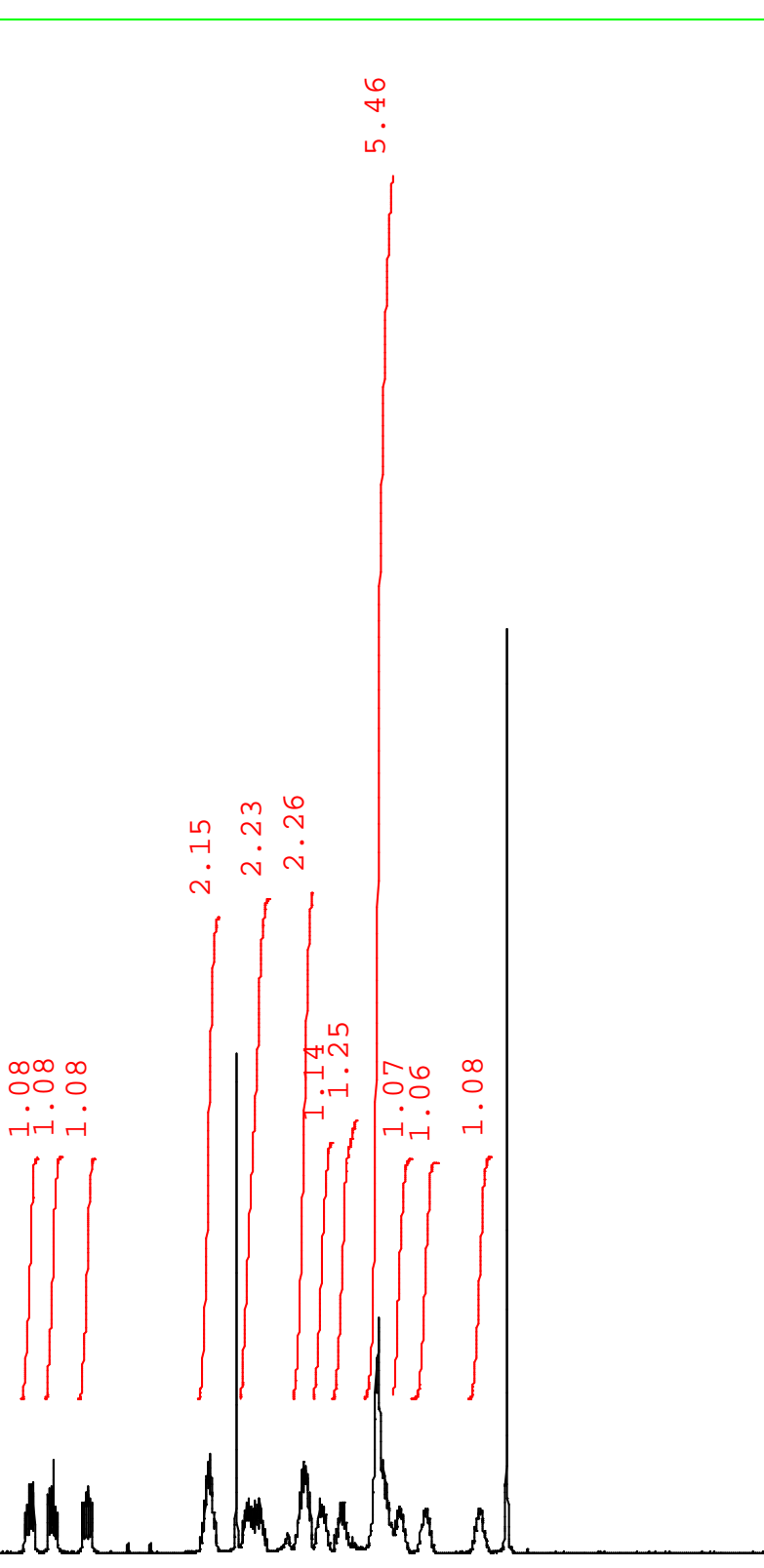

MENUE

OBNUC

OFR

BSET

OBFIN

PW1

DEADT

PREDI

IWT

POINT

SPO

TIMES

DUMMY

FREQU

FLT

ACOTM

$\mathrm{PD}$

ADBIT

RGAIN

$\mathrm{BF}$

T1

$\mathrm{T} 2$

EXMOD

EXPCM

IRNUC

IFR

IRSET

I RF IN

RRPW

IRATN

DFILE SF

LKSET

LKFIN

LKLEV

LGAIN

KPHS

LKS I G

CSPED

FILDC

FILDF
$500.16 \mathrm{MHz}$ $2.41 \mathrm{KHz}$

$6.0 \mathrm{~Hz}$

5.8 us

0.0 us

$0.0000 \mathrm{~ms}$

$1.0 \mathrm{sec}$

13107

13107

16

$7507.4 \mathrm{~Hz}$

$38000 \mathrm{~Hz}$

13.2 us

$1.746 \mathrm{sec}$

$4.000 \mathrm{sec}$

16.00

2000000

$0.12 \mathrm{~Hz}$

0.00

0.00

90.00

100.00

single_pulse.ex2

$0.00 \mathrm{MHz}$

$0.00 \mathrm{KHz}$

$0.0 \mathrm{~Hz}$

60 us

: $\backslash \check{Z} \grave{A} \in E f m \bullet[f g N o .1 \backslash 141 ` 160 \backslash k 0$

$$
\begin{array}{rl}
70.30 & \mathrm{KHz} \\
33.1 & \mathrm{~Hz} \\
0 & \\
0 & \\
0 & \\
0 & \\
0 & \mathrm{~Hz}
\end{array}
$$


DATIM Sat Jun 14 20:38:13 2003 MENUF NON

OBNUC $1 \mathrm{H}$

OFR

OBSET

OBFIN $\quad 5800.0 \mathrm{~Hz}$

DEADT

PREDL

IWT

POINT

SPO

TIMES

DUMMY

FREQU

FLT

DELAY

ACOTM

$\mathrm{PD}$

ADBIT

RGAIN

$\mathrm{BF}$

$\mathrm{T} 1$

T2

T 4

EXMOD

EXPCM

I RNUC

IFR

IRSET

IRF IN

I RRPW

IRATN

DF ILE

$S F$

LKSET

IKFIN

LKLEV

LGAIN

LKP HS

LKSIG

CSPED

FILDC

PM FITDE
5.6 us

108.3 us

$1.0 \mathrm{sec}$

16384

16384

32

$5405.4 \mathrm{~Hz}$ $2700 \mathrm{~Hz}$

3.031 sec

$3.967 \mathrm{sec}$

16.00

$0.12 \mathrm{~Hz}$

0.00

0.00

90.00

100.00

NON

NON:Single.coupled:PW1_ACQTI

$270.05 \mathrm{MHz}$
$112.00 \mathrm{KHz}$

$5800.0 \mathrm{~Hz}$

50 us

511

F: $\backslash \mathrm{EX} .1-100 \backslash \mathrm{K} 021-53 \mathrm{~A} 1$ _PROTON TH5

$$
\begin{array}{rl}
64.60 & \mathrm{KHz} \\
59.0 & \mathrm{~Hz} \\
200 & \\
25 & \\
256 &
\end{array}
$$

$13 \mathrm{~Hz}$

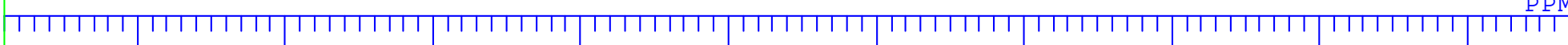




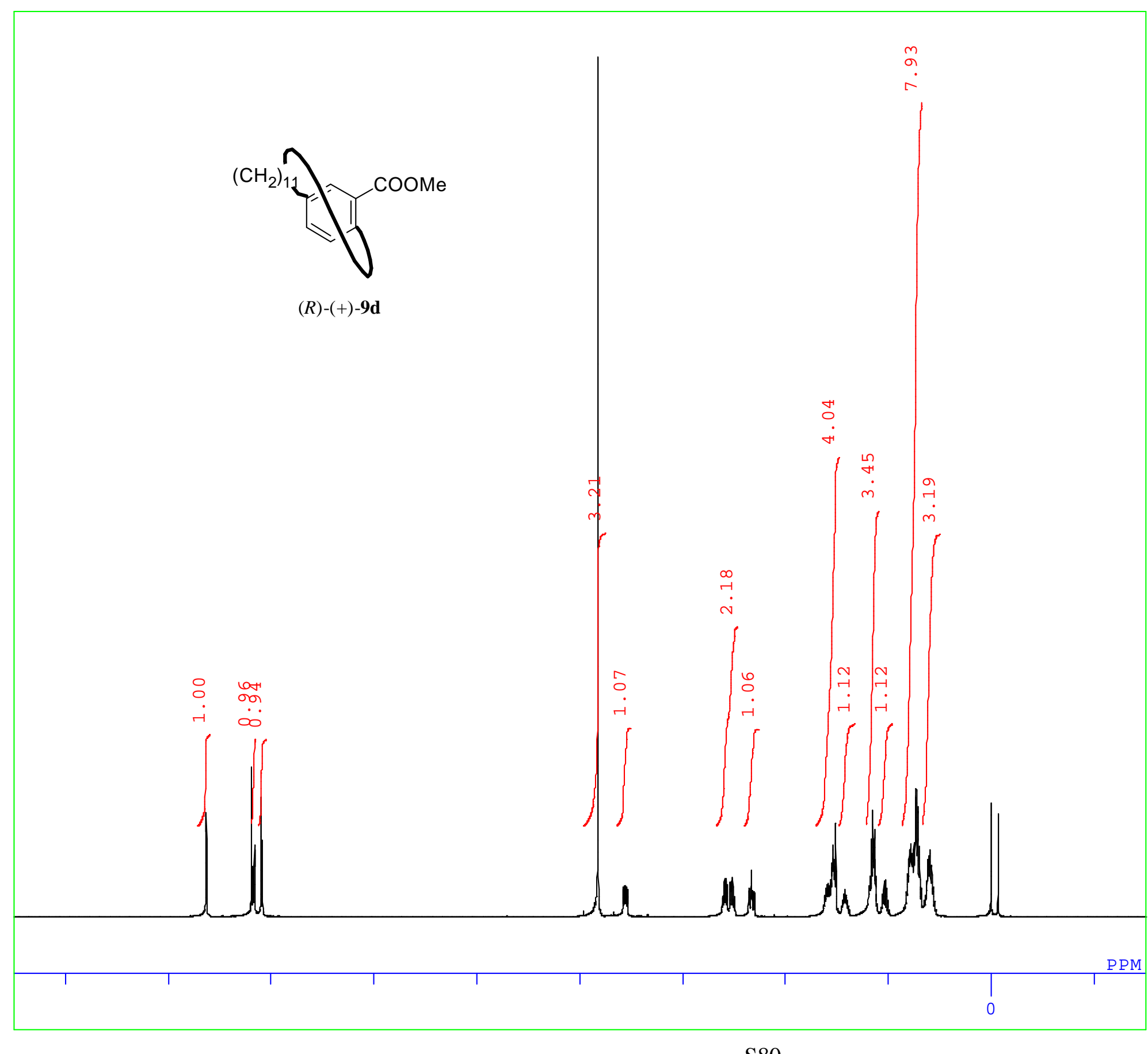

DAT IM

MENUF

OBNUC

OFR

BSE T

OBFIN

PW1

DEADT

PREDL

IWT

POINT

SPO

T IMES

DUMMY

FREQU

FLT

DELAY

ACOTM

PD

ADBIT

RGAIN

$\mathrm{BF}$

T 1

$\mathrm{T} 2$

T 4

EXMOD

EXPCM

IFR

IRSET

IRF IN

RRPW

IRATN

DF I LE

$\mathrm{F}: \backslash \mathbb{E}^{3} f \mathrm{f} \bullet\left[f^{\wedge} \backslash\right.$ K025-169-C_PROTO] 


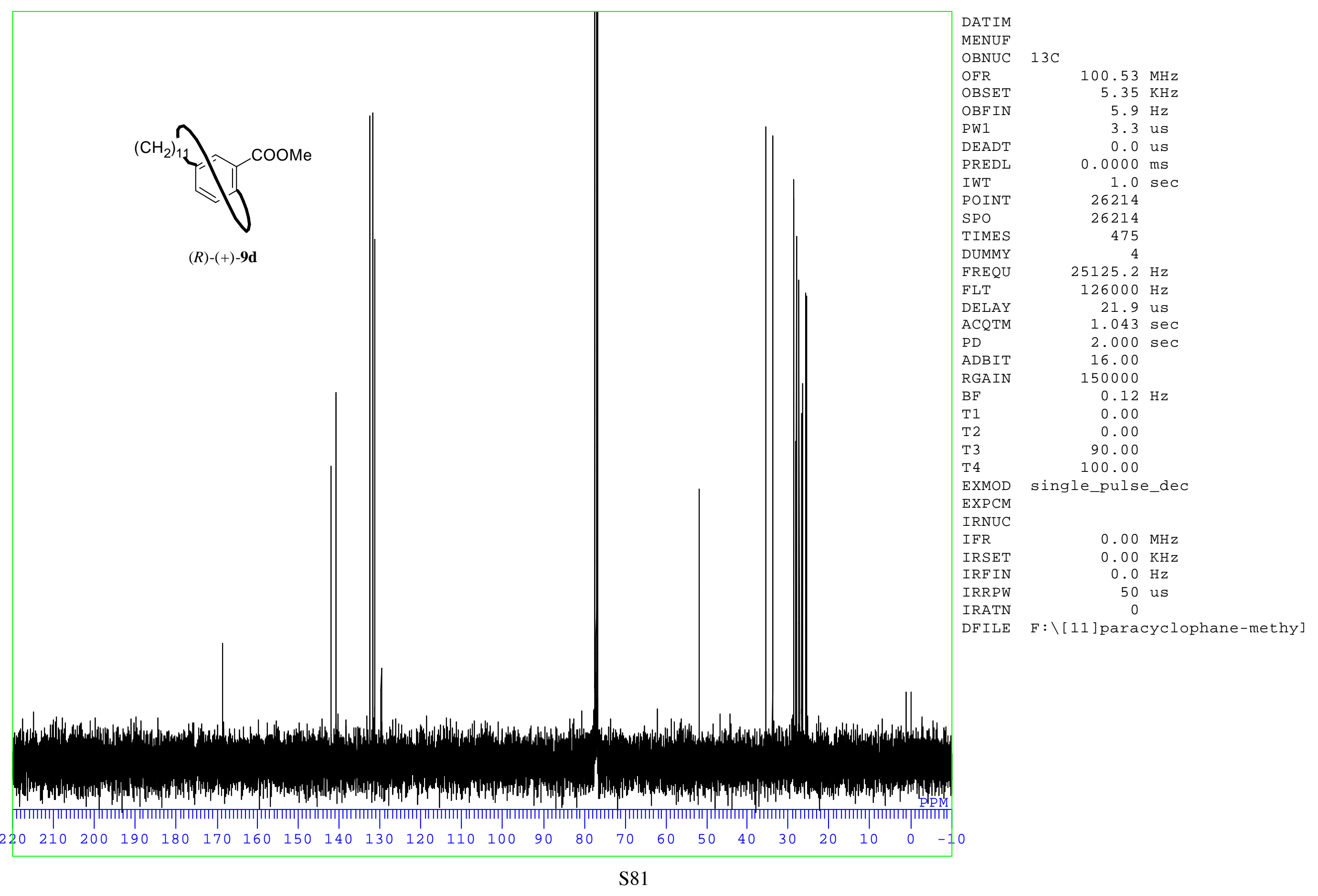

